\title{
Front Fluctuations in One Dimensional Stochastic Phase Field Equations
}

\author{
L. Bertini*, S. Brassesco ${ }^{\dagger}$, P. Buttà ${ }^{\ddagger}$ and E. Presutti ${ }^{\ddagger}$
}

\begin{abstract}
We consider a conservative system of stochastic PDE's, namely a weakly coupled, one dimensional phase field model with additive noise. We study the fluctuations of the front proving that, in a suitable scaling limit, the front evolves according to a non-Markov process, solution of a linear stochastic equation with long memory drift.
\end{abstract}

\section{Part I. Introduction}

\section{General setting, model, and results}

The term "sharp interface limit" denotes a scaling procedure aimed at the derivation of interfaces as geometric objects, e.g. surfaces of codimension one with bounded variation, that is, enough regular for the area measure to be well defined. Of course this makes only sense in the context of systems which undergo phase transitions and of states where different phases coexist. In the limit the other degrees of freedom are lost and we are left with the interface alone. Rigorous proofs are hard, yet a great variety of models has been successfully worked out. The mathematics involved is correspondingly rich, e.g. the theory of $\Gamma$-convergence (to study the sharp interface limit of Ginzburg-Landau like free energy functionals in relation with the equilibrium shape of the interface, as in the Wulff problem) and correspondingly the theory of Gibbsian large deviations (to study the same problems at the more microscopic level of statistical mechanics); singular limit in PDE's, like in the Allen-Cahn, Cahn-Hilliard and phase field equations, and correspondingly, at the microscopic level, hydrodynamic limits of spin or particle systems.

This paper deals with fluctuations. Here again the questions are, first, whether in a sharp interface limit the system is described by a [fluctuating] interface with closed equations of motion and, secondly, the nature of such equations. The problem greatly simplifies in one space dimension where the limit interface is represented by a point which separates the two phases (one to its left and the other one

\footnotetext{
*Partially supported by Cofinanziamento MURST 1999.

$\dagger$ Partially supported by agreement CONICIT-CNR, and Proyecto F-97 000004 (CONICIT).

$\ddagger$ Partially supported by Cofinanziamento MURST 1999 and by NATO Grant PST. CLG. 976552.
} 
to its right). Indeed, until now, most fluctuation results are restricted to $d=1$. Such a state is marginally stable (w.r.t. displacements of the interfaces, see Section 2 for a precise statement) while it becomes stable when there is a conservation law (which imposes that the mass of each phase is preserved). By the conservation law, each displacement of the interface must then necessarily come with a deformation of the profile, hence the intuition that other degrees of freedom may then enter into play. This is confirmed, but in a sense also infirmed, by our results: indeed we will see that extra degrees of freedom become relevant, but their effect can be represented in the limit by terms which depends on the previous history of the interface evolution.

The system we consider is a phase field equation with additive stochastic noise, see (1.1)-(1.2) below. Without noise the interface is given by a special instantonic profile connecting the two phases; in the presence of noise, after suitable rescalings, the limit state is still represented by the same instantonic profile which is however randomly displaced. The displacement obeys an ordinary stochastic equation driven by a white noise forcing term and with a long memory drift, whose effect is to force the interface back toward its initial position, thus restoring the equilibrium of the two coexisting phases.

The evolution is defined by the following stochastic equations

$$
\begin{aligned}
& d m(t)=\left\{\frac{1}{2} \Delta m(t)-V^{\prime}(m(t))+\lambda h(t)\right\} d t+\sqrt{\gamma} d W^{(a)}(t) \\
& d[h(t)+m(t)]=\frac{1}{2} \Delta h(t) d t
\end{aligned}
$$

where the unknowns, $m(t)=m(t, x), h(t)=h(t, x),(t, x) \in \mathbb{R}_{+} \times \mathbb{R}$, are two scalar random fields. In (1.1), $\lambda$ and $\gamma$ are positive parameters; $\Delta$ is the Laplacian on $\mathbb{R}$ and $W^{(a)}(t)$ is a white noise with a cutoff in the spatial covariance. This means $W^{(a)}(t)$ is the canonical process in the filtered probability space $\left(\Omega, \mathcal{F}, \mathcal{F}_{t}, \mathbb{P}\right)$ where $\Omega:=C\left(\mathbb{R}_{+} ; \mathcal{S}^{\prime}(\mathbb{R})\right)\left(\mathcal{S}^{\prime}(\mathbb{R})\right.$ the space of tempered distributions $), \mathcal{F}$ its Borel $\sigma-$ algebra, $\mathcal{F}_{t}$ the canonical filtration, and $\mathbb{P}$ the Gaussian measure with mean zero and covariance

$$
\mathbb{E}\left(\left\langle W^{(a)}(t), \varphi\right\rangle\left\langle W^{(a)}\left(t^{\prime}\right), \varphi^{\prime}\right\rangle\right)=t \wedge t^{\prime}\left\langle\varphi, a_{\gamma}^{2} \varphi^{\prime}\right\rangle, \quad a_{\gamma}(x):=a\left(\gamma^{\beta / 2} x\right)
$$

where $t \wedge t^{\prime}:=\min \left\{t, t^{\prime}\right\},\langle\cdot, \cdot\rangle$ denotes the inner product in $L_{2}(\mathbb{R}, d x)$ as well as the canonical pairing between $\mathcal{S}$ and $\mathcal{S}^{\prime}$. Above $\beta>0$ and $a \in C_{0}^{2}(\mathbb{R})$, i.e. a twice differentiable function with compact support. We assume the normalization $a(0)=1$. Finally $V^{\prime}(m)$ is the derivative of a symmetric, smooth double well potential $V(m)$; for simplicity we assume

$$
V(m)=\frac{m^{4}}{4}-\frac{m^{2}}{2} .
$$

Note we are omitting to write explicitly the dependence on the randomness $\omega \in \Omega$. This will be done throughout the whole paper without further mention. 
In a companion paper, [2], we prove global existence and uniqueness in a space of Hölder continuous functions for the system

$$
\begin{array}{r}
m(t)=p_{t} m(0)+\int_{0}^{t} d s p_{t-s}\left[-V^{\prime}(m(s))+\lambda h(s)\right]+\sqrt{\gamma} \int_{0}^{t} p_{t-s} d W^{(a)}(s) \\
h(t)+m(t)=p_{t}[h(0)+m(0)]-\frac{1}{2} \int_{0}^{t} d s\left(\Delta p_{t-s}\right) m(s)
\end{array}
$$

where $p_{t}=e^{t \Delta / 2}$ is the heat semigroup, namely the integral operator with kernel

$$
p_{t}(x, y)=\frac{e^{-(x-y)^{2} / 2 t}}{\sqrt{2 \pi t}}
$$

Observe that the integral on the r.h.s. of (1.6) is well defined because $m(s)$ is Hölder continuous. The system (1.1)-(1.2) is defined in terms of the integral equations (1.5)-(1.6) and called "stochastic phase field equations".

General background and physical interpretation are discussed in the sequel, here we proceed by stating our main result, presented in the next theorem. We consider the initial condition

$$
m(0)=\bar{m}, \quad h(0)=0
$$

where

$$
\bar{m}_{\xi}(x):=\tanh (x-\xi), \quad \bar{m}:=\bar{m}_{0}
$$

is a standing wave (that we call "instanton") with "center" $\xi \in \mathbb{R}$. We suppose that

$$
\lambda=\sqrt{\gamma}
$$

and denote by $\left(m^{(\lambda)}(t), h^{(\lambda)}(t)\right)$ the solution of (1.1)-(1.2) with (1.10) and initial condition (1.8). Our main result is

Theorem 1.1 There exists a process $x^{(\lambda)}(t)$ in $C\left(\mathbb{R}_{+}\right)$, adapted to $\mathcal{F}_{t}$, such that for each $\tau, \varepsilon>0$

$$
\lim _{\lambda \downarrow 0} \mathbb{P}\left(\sup _{t \leq \lambda^{-2} \tau}\left\|m^{(\lambda)}(t)-\bar{m}_{x^{(\lambda)}(t)}\right\|_{\infty}>\varepsilon\right)=0 .
$$

Furthermore, denoting weak convergence in $C\left(\mathbb{R}_{+}\right)$by $\Longrightarrow$ and defining, after scaling, $x_{\lambda}(\tau):=x^{(\lambda)}\left(\lambda^{-2} \tau\right)$, we have that $x_{\lambda} \Longrightarrow x$ as $\lambda \downarrow 0$, where $x(\tau)$ is the unique solution of

$$
x(\tau)=b(\tau)-3 \int_{0}^{\tau} d s \frac{x(s)}{\sqrt{2 \pi(\tau-s)}}
$$

in which $b(\tau)$ is a one dimensional Brownian motion with diffusion coefficient $D=3 / 4$. 
The coefficient 3 in (1.12) and the value $D=3 / 4$ above are related to the specific choice of the potential $V$. Existence and properties of the process solution of (1.12) are discussed in [3].

For the physical interpretation, we start from the equation without noise and with $\lambda=0$, namely (1.1) with $\lambda=\gamma=0$. This is the well known Allen-Cahn (AC) equation with double well potential $V(m)$, which arises as the gradient flow associated to the Ginzburg-Landau free energy functional

$$
\mathcal{F}(m)=\int_{\mathbb{R}} d x\left\{\frac{1}{4}|\nabla m(x)|^{2}+V(m(x))\right\}
$$

$(\mathcal{F}$ decreases along the solutions of the AC equation). The minimizers of $\mathcal{F}(m)$ are the two constant functions $m_{+}(x)=1$ and $m_{-}(x)=-1$, therefore the values of the order parameter $m= \pm 1$ correspond to pure phases and the interface for (1.13) is (up to translations) the profile $m(x)$ which minimizes $\mathcal{F}(m)$ under the condition that asymptotically as $x \rightarrow \pm \infty$ it converges to \pm 1 . The associated Euler-Lagrange equation is the stationary AC equation

$$
\frac{1}{2} \Delta m-V^{\prime}(m)=0
$$

which, imposing the above conditions at $\pm \infty$, has the instanton $\bar{m}$ of (1.9) as its unique solution (modulo translations). Therefore $\bar{m}_{\xi}$ is the equilibrium state which has the two phases coexisting to the right and to the left of $\xi$, it represents the "mesoscopic interface" with $\xi$ its "mesoscopic location" (mesoscopic instead of macroscopic because the interface is "diffuse" and the transition from one phase to the other, even though exponentially fast, is not sharp; mesoscopic instead of microscopic because the AC equation and the Ginzburg-Landau functional can be derived by a scaling procedure from particle or spin systems, i.e. from an underlying more microscopic structure).

The next step is with $\lambda>0$, but still $\gamma=0$. Then (1.1) is coupled to (1.2) and the two together give an example of phase field equations (PFE). Here $h$ is a thermodynamic potential conjugated to the order parameter $m$ : if $m$ is a magnetization density, then $h$ is a magnetic field, our notation is inspired by such an interpretation. More commonly however, $m$ is a relative concentration of one species in a binary alloy and $h$ is a relative temperature. The effective potential will then depend on the relative temperature $h$, our choice is simply to replace $V(m)$ by $V(m)-h m$. At the critical temperature, which corresponds to $h=0$, the alloy can exist in two different concentrations $m= \pm 1$, but, as the temperature $h$ changes, the equilibrium concentrations vary, one becomes stable and the other one metastable. In the presence of a given temperature profile $h$, the $\mathrm{AC}$ rate of change of the concentration density at $x$ at time $t$, i.e. $d m(t, x) / d t$, is given by (1.1) (with $\gamma=0$ ). Due to latent heat, there is a corresponding change of temperature given (in the proper units) by $d h(t, x) / d t=-d m(t, x) / d t$. Simultaneously, by the 
Fourier law, the temperature diffuses according to the r.h.s. of (1.2). This has a feedback in (1.1) so that (1.1) and (1.2) are coupled. In conclusion the PFE describe a change of phase including latent heat effects and, because of that, unlike for the $\mathrm{AC}$ equation, there is a conservation law: the integral of $m+h$ is in fact invariant under PFE. The noise term in (1.1) takes into account some external fluctuating force and the resulting equation is known in the physical literature as model $\mathrm{C}$ of Hohenberg and Halperin, [14].

Since $\bar{m}$ solves (1.14), the state $m=\bar{m}, h=0$ is a stationary solution of $\mathrm{PFE}$, which is therefore interpreted, like in $\mathrm{AC}$, as the mesoscopic interface. We are then studying in Theorem 1.1 what happens to the interface when there are small (because $\gamma \rightarrow 0$ in Theorem 1.1), external perturbations which produce a random change $\sqrt{\gamma} d W^{(a)}(t)$ of magnetization (or, in the other interpretation, of concentration), the analysis including latent heat effects.

The small parameter $\gamma$ in the noise term has the meaning of a ratio between mesoscopic and microscopic space units, the former referring to (1.1), the latter to some microscopic model, as for instance the Glauber + Kawasaki process introduced in [12]. A formal comparison with the microscopic model in [12] would lead to a more complex structure for the additive noise; however we stick to (1.1) (which catches the correct behavior of critical fluctuations, see [5]) to make our analysis simpler. In conclusion the scaling $\gamma \rightarrow 0$ has a natural justification in terms of the microscopic origin of the noise term, the scaling of time in Theorem 1.1 is on the other hand justified a posteriori: it is the correct scaling for observing finite displacements of the interface. On the contrary, the equality (1.10) has no clear physical interpretation; it is true that a scaling with $\lambda \rightarrow 0$ is widely used in the PFE literature to stress "kinetic undercooling effects", see [11], [17], but relating $\lambda$ to the noise as in (1.10) is just a matter of technical convenience. We will come back to this in the next section in the paragraph "the role of the assumptions".

\section{Heuristic analysis and outline of proofs}

By Theorem 1.1, with probability going to 1 , the process $m^{(\lambda)}(t), t \leq \lambda^{-2} \tau$, is always close to the manifold of instantons $\mathcal{M}=\left\{\bar{m}_{\xi}, \xi \in \mathbb{R}\right\}$, see (1.11); the theorem then identifies the motion along $\mathcal{M},(1.12)$. It is then natural to describe $m^{(\lambda)}(t)$ in terms of coordinates along and transversal to $\mathcal{M}$, these are the Fermi coordinates that we are going to define.

Stability of instantons, Fermi coordinates. Closeness to $\mathcal{M}$ is a consequence of the stability of $\mathcal{M}$ under the $\mathrm{AC}$ evolution. Under $\mathrm{AC}$, in fact, $\mathcal{M}$ attracts exponentially fast all data which are in a small neighborhood, in sup norm, $\|\cdot\|_{\infty}$, of $\mathcal{M}$ : namely there are $\delta, c$ and $a$ all positive so that if for some $\xi,\left\|m-\bar{m}_{\xi}\right\|_{\infty}<\delta$, then there is a $\xi^{\prime}$ for which, for all $t,\left\|m(t)-\bar{m}_{\xi^{\prime}}\right\|_{\infty} \leq c e^{-a t}, m(t)$ being the solution of the AC equation starting from $m$. This obviously fails if we add noise (thus considering (1.1) with $\lambda=0$ and $\gamma>0$ ), but the noise, in a polynomial scale, cannot drive too far away from $\mathcal{M}$ because of the exponential attraction of 
the deterministic part of the equation: in [10] it is shown that for any $\tau>0$ and $\zeta>0$

$$
\lim _{\gamma \rightarrow 0} \mathbb{P}\left(\sup _{t \leq \gamma^{-1} \tau} \operatorname{dist}(m(t), \mathcal{M}) \geq \gamma^{1 / 2-\zeta}\right)=0
$$

where "dist" denotes distance in sup norm. The analysis extends to our case with $\lambda=\sqrt{\gamma}$ and (1.1)-(1.2) coupled, as stated in (1.11) and proved in Section 8.

Going back to $\mathrm{AC}$ without noise, observe that stability of $\mathcal{M}$ does not mean stability of the single instanton: let $m$ be a small deviation from $\bar{m}_{\xi}$, then from what we said above it will relax under AC to some $\bar{m}_{\xi^{\prime}}$, with $\xi^{\prime}$ close but not necessarily equal to $\xi$. In the space of all profiles $m, \bar{m}_{\xi}$ is marginally stable along the direction $\mathcal{M}$ while all the other directions are stable. It is then natural to associate to each $m$ (as above) the value $\xi^{\prime}$ of the center of the limit instanton. In practice, however, it is better to work with a more "geometrical" definition. Following [10,9], we define a center $\xi$ of $m$ as a real number which minimizes the $L_{2}$-norm of $m-\bar{m}_{x}$ (as a function of $x$ ). Then $\xi$ is such that

$$
\left\langle m-\bar{m}_{\xi}, \bar{m}_{\xi}^{\prime}\right\rangle=0 \text {. }
$$

The center $\xi$ has also a dynamical interpretation. Let $L_{\xi}$ be the operator

$$
L_{\xi} v=\frac{1}{2} \Delta v+\left(1-3 \bar{m}_{\xi}^{2}\right) v
$$

obtained by linearizing the AC equation around $\bar{m}_{\xi}$. It is readily seen that $L_{\xi}$ is self-adjoint in $L_{2}(\mathbb{R}, d x)$, it has eigenvalue 0 with eigenvector $\bar{m}_{\xi}^{\prime}$ while the rest of the spectrum is on the negative axis strictly away from 0 . Then, if $m$ has center $\xi$, the deviation $v=m-\bar{m}_{\xi}$ by (2.2) has no component along $\bar{m}_{\xi}^{\prime}$, hence the linearized evolution starting from $v$ decays exponentially fast and correspondingly $m$ converges to $\bar{m}_{\xi}$, so that the center of $m$ is also the center of the instanton to which the linearized AC evolution converges. The pair $\left\{\xi, m-\bar{m}_{\xi}\right\}$ is known as the Fermi coordinates of $m$.

This notion of a center of a function plays an important role also in our proofs, so we will spend a few more words, recalling Proposition 3.2 in [9], which gives a sufficient condition for $m=m(x)$ to have a center.

Proposition 2.1 There is a constant $\delta>0$ such that if there exists $x_{0} \in \mathbb{R}$ so that $\left\|m-\bar{m}_{x_{0}}\right\|_{\infty} \leq \delta$ then the following holds for some constant $C=C(\delta)$ independent of $x_{0}$ and $m$.

(i) The function $m$ has a unique center $x$ and

$$
\left|x-x_{0}\right| \leq C\left\|m-\bar{m}_{x_{0}}\right\|_{\infty}
$$

(ii) the center $x$ has the expansion

$$
\begin{array}{r}
x=x_{0}-\frac{3}{4}\left\langle\bar{m}_{x_{0}}^{\prime}, m-\bar{m}_{x_{0}}\right\rangle-\frac{9}{16}\left\langle\bar{m}_{x_{0}}^{\prime}, m-\bar{m}_{x_{0}}\right\rangle\left\langle\bar{m}_{x_{0}}^{\prime \prime}, m-\bar{m}_{x_{0}}\right\rangle+R\left(m-\bar{m}_{x_{0}}\right), \\
\left|R\left(m-\bar{m}_{x_{0}}\right)\right| \leq C\left\|m-\bar{m}_{x_{0}}\right\|_{\infty}^{3} . \quad(2.5)
\end{array}
$$


Thus, by (2.1) and (1.11) and with the help of Proposition 2.1, we can talk unambiguously, with probability going to 1 , of the center $\xi(t)$ of $m(t)$ both for the stochastic AC equation and PFE. This gives us a precise definition of the location of the interface even without sharp interface limits; we will prove convergence to (1.12) by studying the asymptotic law of $\xi(t)$ as $\gamma \rightarrow 0$.

Heuristics of the AC equation and PFE with noise. We start from the simpler (and instructive) case of the AC equation with noise, i.e. (1.1) with $\lambda=0$ but with $\gamma>0$. This is well studied in the literature, [9, 10, 13], even though in slightly different contexts. The scaling procedure is the same as in Theorem 1.1 and it leads to the same limit law but without drift, i.e. a Brownian motion. To explain heuristically the result, let us regard the forcing term $\sqrt{\gamma} d W^{(a)}(t)$ as a "source of small kicks" which we decompose in a component along $\mathcal{M}$ and another one orthogonal to $\mathcal{M}$. The latter fights against the $\mathrm{AC}$ drift which pushes back toward $\mathcal{M}$, and because of the small factor $\sqrt{\gamma}$, to a first order, we forget about orthogonal components. On the contrary the kicks along $\mathcal{M}$ are not contrasted and they sum up: thus we are approximating

$$
d m(t) \approx \frac{3}{4} \sqrt{\gamma} \bar{m}_{\xi(t)}^{\prime}\left\langle\bar{m}_{\xi(t)}^{\prime}, d W^{(a)}(t)\right\rangle, \quad\left\langle\bar{m}^{\prime}, \bar{m}^{\prime}\right\rangle=\frac{4}{3}
$$

where $\xi(t)$ is the center of $m(t)$. Also $m(t) \approx \bar{m}_{\xi(t)}$, hence

$$
d m(t) \approx \bar{m}_{\xi(t)}^{\prime} d \xi(t)
$$

and, in conclusion,

$$
d \xi(t) \approx \frac{3}{4} \sqrt{\gamma}\left\langle\bar{m}_{\xi(t)}^{\prime}, d W^{(a)}(t)\right\rangle
$$

namely $\xi\left(\gamma^{-1} t\right)$ is a Brownian motion with diffusion $3 / 4$, which is what is proved in [10].

The argument for the system (1.1)-(1.2) is similar, the only difference from the stochastic AC equation in (1.1) lies in the simple, innocent looking term $\lambda h$, which is however the source of all problems. The same heuristics leading to (2.6) applies to (1.1) by simply adding $\lambda h(t)$ to the noise; writing $\sqrt{\gamma}=\lambda$ according to (1.10), we then get

$$
d m^{(\lambda)}(t) \approx \frac{3}{4} \lambda \bar{m}_{\xi(t)}^{\prime}\left\{\left\langle\bar{m}_{\xi(t)}^{\prime}, h(t)\right\rangle d t+\left\langle\bar{m}_{\xi(t)}^{\prime}, d W^{(a)}(t)\right\rangle\right\}
$$

and, using (2.7),

$$
d \xi(t) \approx \frac{3}{4} \lambda\left\{\left\langle\bar{m}_{\xi(t)}^{\prime}, h(t)\right\rangle d t+\left\langle\bar{m}_{\xi(t)}^{\prime}, d W^{(a)}(t)\right\rangle\right\} .
$$

On the other hand, writing (1.2) in integral form and recalling that $h(0)=0$, we have

$$
h(t)=-\int_{0}^{t} p_{t-s} d m^{(\lambda)}(s)
$$


Using again (2.7) and taking the scalar product with $\bar{m}_{\xi(t)}^{\prime}$ we get from (2.11)

$$
\left\langle\bar{m}_{\xi(t)}^{\prime}, h(t)\right\rangle \approx-\int_{0}^{t}\left\langle\bar{m}_{\xi(t)}^{\prime}, p_{t-s} \bar{m}_{\xi(s)}^{\prime}\right\rangle d \xi(s) .
$$

The approximate system $(2.10)-(2.12)$ (with $\approx$ replaced by equality) is not as easy to study as the one which approximates the stochastic AC equation, but it can be seen to give the correct result (1.12) for the limit motion of the center, [3]. To see the relation with (1.12) we make another approximation whose validity will be justified in the course of the proof of Theorem 1.1. The approximation consists in replacing $\bar{m}_{\xi(t)}^{\prime}$ by $\bar{m}^{\prime}$ (i.e. $\xi(t) \rightarrow 0$ ) in the scalar products in (2.10) and (2.12) (the reason for its validity is that the displacements of the center are finite while the field $h$ becomes flat because the diffusion in (1.2) acts for long times). The new system is then (forgetting about the noise cutoff)

$$
\begin{gathered}
d \xi(t)=\frac{3}{4} \lambda\left\langle\bar{m}^{\prime}, h(t)\right\rangle+\lambda d b(t) \\
\left\langle\bar{m}^{\prime}, h(t)\right\rangle=-\int_{0}^{t}\left\langle\bar{m}^{\prime}, p_{t-s} \bar{m}^{\prime}\right\rangle d \xi(s)
\end{gathered}
$$

where $b(t)$ is the Brownian motion with diffusion $3 / 4$ of Theorem 1.1. Using (2.14) to rewrite the first term on the r.h.s. of (2.13) we get

$$
\xi(t)=\lambda b(t)-\frac{3}{4} \lambda \int_{0}^{t} d s \int_{0}^{s}\left\langle\bar{m}^{\prime}, p_{s-s^{\prime}} \bar{m}^{\prime}\right\rangle d \xi\left(s^{\prime}\right) .
$$

Integrating by parts, after some simple algebra,

$$
\xi(t)=\lambda b(t)-\frac{3}{4} \lambda \int_{0}^{t} d s^{\prime} \xi\left(s^{\prime}\right)\left\langle\bar{m}^{\prime}, p_{t-s^{\prime}} \bar{m}^{\prime}\right\rangle .
$$

Approximating

$$
\left\langle\bar{m}^{\prime}, p_{t-s^{\prime}} \bar{m}^{\prime}\right\rangle=\int d x \int d y \bar{m}^{\prime}(x) p_{t-s^{\prime}}(x, y) \bar{m}^{\prime}(y) \approx \int d x \int d y \frac{\bar{m}^{\prime}(x) \bar{m}^{\prime}(y)}{\sqrt{2 \pi\left(t-s^{\prime}\right)}}
$$

and recalling that $\int \bar{m}^{\prime}=2,(2.15)$ becomes (1.12), in the above approximation, which can be made rigorous in the limit $\lambda \rightarrow 0$, having set $t=\lambda^{-2} \tau$.

Main difficulties and outline of proof. The heuristic arguments outlined above are essentially based on a linear approximation, their validity therefore rests on a rigorous proof that the non linear effects are negligible. Since the strength of the noise is $\sqrt{\gamma}$, we cannot hope to improve the a priori bounds beyond $\| m(t)-$ $\bar{m}_{\xi(t)} \|_{\infty} \approx \sqrt{\gamma}$. Then the non linear terms which are, to lowest order, quadratic have order $\gamma$; since they act for a time $\lambda^{-2} \tau$ (that is $\gamma^{-1} \tau$ ), a naive estimate gives a non-negligible contribution. The fact that they are indeed negligible must then 
come from cancellations, and the true problem is to find them and take them into proper account.

This is more clearly seen in the stochastic AC equation. Following [10], we split the time axis into intervals of length $T$, taking $T$ long, yet very small as compared to the macroscopic time $\gamma^{-1} \tau$, for instance $T=\gamma^{-1 / 10}$. The crucial point is an iterative procedure for which the problem reduces to the analysis of only one of these intervals, a clear advantage, because in such a "short time" the non linear effects are under control. Couplings are used for this crucial reduction. We compare in fact, in the generic interval $[n T,(n+1) T]$, the true process $m(t)$ which starts from $m(n T)$ and the new process $\hat{m}(t)$ which starts from $\bar{m}_{\xi(n T)}$, $\xi(n T)$ the center of $m(n T)$, the coupling is simply to use for both processes the same noise $\sqrt{\gamma} d W^{(a)}(t)$. Under the assumption that $\left\|m(n T)-\bar{m}_{\xi(n T)}\right\|_{\infty} \leq \gamma^{1 / 2-\zeta}$, see (2.1), it can be seen that, with probability going to 1 as $\gamma \rightarrow 0, \| m((n+1) T)-$ $\hat{m}((n+1) T) \|_{\infty} \leq C \gamma^{1-2 \zeta},(C$ a constant $)$. By Proposition 2.1 , the displacements of the center in the interval $[n T,(n+1) T]$, as computed with the two evolutions, differ proportionally to $\gamma^{1-2 \zeta}$ : since the number of intervals grows proportionally to $\gamma^{-1} \tau / T$, the sum of all these differences goes like $\gamma^{1-2 \zeta} \gamma^{-1} \tau / T$, which vanishes, after choosing $2 \zeta<1 / 10$. We can then study in each interval the process which starts from an instanton. Neglecting for simplicity the cutoff on the noise (with the cutoff some extra computations are needed), then the displacement of the center in a time interval $T$ does not depend on the initial center and it is therefore independent of the past. The displacements of the centers (each time restarting from an instanton) are thus independent variables with mean 0 (by the symmetry between right and left) and, using classical arguments on convergence to Brownian motion, in the end, we need to sum their variances; since we are already with squares, it turns out that the linear approximation is sufficiently accurate and this explains the validity of the linear approximation in the previous heuristic analysis.

While the above approach works well in the stochastic AC equation, Theorem 1.1 tell us that it fails, as there are long memory effects in the limit law. More bad news: the last term in (1.12), responsible for these effects, according to the heuristic analysis of the previous paragraph, comes from the term $\lambda h(t)$ and since it produces a finite drift in a time $\lambda^{-2}$, the order of magnitude of $h(t)$ must be $\approx \lambda$. Therefore we need an accuracy of order $\lambda$, which is comparable with the deviations of $m^{(\lambda)}$ from $\mathcal{M}$ (recall $\lambda=\sqrt{\gamma}$ ) that has been neglected so far.

Our approach to the problem, since when we began the present work, has been "to trust" [10] and to consider the non linear terms that are left as being negligible when we linearize (1.1) around $\bar{m}_{\xi(n T)}, \xi(n T)$ the center of $m^{(\lambda)}(n T)$. It is evidently not possible to use the coupling argument of [10], yet in some maybe more complicated way, in the end we "must" see that they are not relevant. The extra term $\lambda h(t)$ is new w.r.t. [10] and has to be dealt anew. According to the final result and for what said before, we expect $h(t) \approx \lambda$, but let us even suppose, pessimistically, that $h(t)$ is of the order of unity. Its effect for a time $T$ will then be of order $\lambda T$, hence still infinitesimal. Moreover, by $(2.11), h(t)$ can be large only if $d m^{(\lambda)}(s)$ is large, but $d m^{(\lambda)}(s)$ is under control except for the term $\lambda h(s)$. Due 
to the presence of the small factor $\lambda$ this gives a virtuous feedback which allows to control the magnitude of $h(t)$.

This is done in Sections 3 and 4 . We first write down an integral equation, (3.7), for $v^{(n)}(t)=m^{(\lambda)}(t)-\bar{m}_{\xi(n T)}$, the superscript $n$ recalls that we are considering $t \in[n T,(n+1) T] . v^{(n)}(t)$ is written as the sum of 8 terms, two of them, called $\Gamma_{2}^{(n)}(t)$ and $\Lambda_{3}^{(n)}(t)$ are the important ones, which give contributions to the limit, respectively the Brownian motion and the drift. All the others vanish, but at this stage this is not yet established as they depend on the unknown $m^{(\lambda)}(t)$. In Section 4 we study by iterations the integral equation (3.7) to prove bounds on $v^{(n)}(t)$ and on the displacements of the center. We derive in this way (1.11) and establish even sharper bounds that will be used in the successive proofs.

The proof of the convergence to (1.12) is reported in the remaining sections. We begin Section 5 by splitting the term $\Lambda_{3}^{(n)}(t)$ into the sum of 3 other terms, see (5.1)-(5.5), which foresees the approximation done in (2.16). We then use (2.5) to deduce an equation for the displacements of the center, which are called $\psi_{n}$, see (5.7). The equation is (5.9), which is a sort of linear integral equation in the $\psi_{n}$ with kernel $A_{n, k}, k<n$, and known data $\eta_{n}$ : "sort of" because the $\eta_{n}$ still depend on the unknowns $v^{(n)}(\cdot)$. The elements $A_{n, k}$ decay as $(n-k)^{-1 / 2}$. It is then convenient to reduce to the matrix $A^{2}$, so that we iterate once (5.9) obtaining (5.17), where the kernel is now $A_{n, k}^{2}$ and the "known terms" are $\eta_{n}$ and $(A \eta)_{n}$.

In Section 6 we study these "known terms" which are splitted into four groups. The first one consists of truly known terms which survive in the limit (they come from $\Gamma_{2}^{(n)}(t)$ ). The terms in the second group, which instead may depend on $v^{(n)}(t)$, are all directly proved to be negligible using the a priori bounds of Section 4 . For those in the third group we cannot proceed in this way, but we need to use the integral equation for $v^{(n)}(t)$ and only after sufficiently many iterations, we can show that they are negligible. Finally, the last group collects terms which become negligible because of stochastic cancellations. The latter are studied in Section 7, the others in Section 6.

We draw the conclusions of our analysis in Section 8 where we complete the proof of Theorem 1.1.

Role of assumptions. We start from the assumption (1.10) which is conceptually the most important one, the others are of a more technical nature. As already remarked, there are several studies of sharp interface limits on PFE where $\lambda$ is scaled to 0 . This describes an intermediate regime (called kinetic undercooling) where thermodynamical equilibrium is not fully reached. Thus our model should be regarded as kinetic undercooling in the presence of stochastic perturbations. As said, the relation between $\lambda$ and $\gamma$ stated in (1.10) does not have a straight physical interpretation, it is just the right way to scale (1.1)-(1.2) and have a nice limit law. One may however wonder what would happen if we took a different relation than (1.10). We have not worked out the details, but we can at least present some educated guess. If we multiply $h(t)$ in (1.1) by a constant $\theta$, we would then derive a limit law with such a factor multiplying the last term in (1.12). Let us then 
consider the scaling behavior of

$$
x(t)=b(t)-3 \theta \int_{0}^{t} d s \frac{x(s)}{\sqrt{2 \pi(t-s)}}
$$

both when $\theta$ vanishes and when it diverges. Set $y(t)=\theta^{a} x\left(\theta^{c} t\right)$, then

$$
y(t)=\theta^{a} \theta^{c / 2} b(t)-3 \theta \theta^{c / 2} \int_{0}^{t} d s \frac{y(s)}{\sqrt{2 \pi(t-s)}} .
$$

Imposing

$$
a+c / 2=0, \quad 1+c / 2=0 ; \quad a=1, \quad c=-2
$$

we have (2.18) equal to (1.12). Thus if we take (1.1) with $\lambda \rightarrow \lambda^{1+\alpha}$ and call $\theta=\lambda^{\alpha}$, we believe that our analysis extends, at least for small $|\alpha|$, and that the center $\xi(t)$ of the solution of the corresponding equation is such that

$$
\lambda^{\alpha} \xi\left(\lambda^{-2-2 \alpha} t\right)
$$

converges in law to $(1.12)$.

Concluding remarks and perspectives. A forthcoming paper, [3], is devoted to the analysis of the limit process (1.12). This can be characterized in terms of a Brownian motion with absorption at the origin, which in turn is reduced to the well studied one dimensional Schrödinger equation with Dirac's delta potentials. A quite explicit expression for the solution of (1.12), is then available, in particular it shows that the displacements $\xi(\tau)$ of the front have typical size $\sqrt{\log \tau}$ for $\tau$ large; "aging phenomena" are also present.

The "cluster fluctuations" have instead the usual Brownian structure. Consider an initial state with $h_{0}=0$ and $m_{0}(x)$ the symmetric function which coincides with $\bar{m}_{-\xi}(x), \xi>0$, for $x \leq 0$. We interpret it as a "plus cluster" in the region $(-\xi, \xi)$ with the minus phase outside. To make it sharp we set $\xi=\lambda^{-1} \ell, \ell>0$, and consider the process (1.1)-(1.2) (always assuming $\lambda=\sqrt{\gamma}$ ). Preliminary results (see also [3]) indicate that, in proper units, the coordinates $\xi_{1}(\tau)$ and $\xi_{2}(\tau)$ of the two centers, evolve in the limit according to a system of two stochastic equations. The system can be diagonalized into the variables $\xi_{G}(\tau):=\left[\xi_{1}(\tau)+\xi_{2}(\tau)\right] / 2$ and $\xi(\tau):=\xi_{1}(\tau)-\xi_{2}(\tau)$. The variable $\xi_{G}(\tau)$ has the law of a Brownian motion, $\xi(\tau)$ is independent of $\xi_{G}(\tau)$ and obeys an equation like (1.12).

We hope to be able to accomplish the above program including the analysis of the case with several clusters (and suitable scalings), the ultimate goal being the study of interface fluctuations in many dimensions. 


\section{Part II. Stability of fronts}

\section{The iterative scheme}

Before starting with the proofs we introduce some notation that will be used throughout the paper:

Notation. $C$ will denote a generic constant whose numerical value may change from line to line (from the statements it will appear clear on which parameters it depends). For $p \in[1, \infty]$ we denote by $\|\cdot\|_{p}$ the norm in $L_{p}(\mathbb{R}, d x)$.

We will study the problem (1.1)-(1.10) by an iterative procedure. To this end, we divide the microscopic time-line $\mathbb{R}_{+}$into intervals $\left[T_{n}, T_{n+1}\right], T_{n}=n T$, where $n \in \mathbb{N}$ and $T=\lambda^{-(1 \wedge \beta) / 20}(\beta$ as in (1.3)). We then associate to any macroscopic time interval $[0, \tau], \tau>0$, the set of microscopic time intervals $\left[T_{n}, T_{n+1}\right]$, with $n \leq$ $n_{\lambda}(\tau)$, where $n_{\lambda}(\tau)$ is the integer part of $\left(\lambda^{2} T\right)^{-1} \tau$, namely $n_{\lambda}(\tau)=\left[\left(\lambda^{2} T\right)^{-1} \tau\right]$. To simplify notation, we write $m(t)=\{m(t, x), x \in \mathbb{R}\}$ and $h(t)=\{h(t, x), x \in \mathbb{R}\}$ for the solution of (1.1)-(1.10) (omitting the dependence on $\lambda$ ).

We next define, by induction on $n \geq 0$, the numbers $x_{n}$ and the functions $v^{(n)}(t)=\left\{v^{(n)}(t, x), x \in \mathbb{R}\right\}, t \in\left[T_{n}, T_{n+1}\right]$. They will have the property that for each $t \in\left[T_{k}, T_{k+1}\right]$

$$
v^{(k)}(t)=m(t)-\bar{m}_{x_{k}} \quad \text { if } \quad\left\|v^{(h)}\left(T_{h+1}\right)\right\|_{\infty} \leq \delta \text { for all } 0 \leq h \leq k-1
$$

with $\delta$ as in Proposition 2.1.

For $n=0$ we set $x_{0}=0$ and $v^{(0)}(t)=m(t)-\bar{m}_{x_{0}}, 0 \leq t \leq T$, so that (3.1) holds. Suppose that, by the induction hypothesis, we have already defined for all $k \leq n-1$ both $x_{k}$ and $v^{(k)}(t)$ and that (3.1) holds for such $k$ 's. If there is $k \leq n-1$ such that $\left\|v^{(k)}\left(T_{k+1}\right)\right\|_{\infty}>\delta$, we set $x_{n}=0$ and $v^{(n)}(t)=0$, $t \in\left[T_{n}, T_{n+1}\right]$. Otherwise we define $x_{n}$ as the center of $\bar{m}_{x_{n-1}}+v^{(n-1)}\left(T_{n}\right)$, which by Proposition 2.1 is well defined, as $\left\|v^{(n-1)}\left(T_{n}\right)\right\|_{\infty} \leq \delta$. We then set, according to (3.1), $v^{(n)}(t)=m(t)-\bar{m}_{x_{n}}, t \in\left[T_{n}, T_{n+1}\right]$, and have (always under the assumption that $\left\|v^{(k)}\left(T_{k+1}\right)\right\|_{\infty} \leq \delta$ for all $\left.k \leq n-1\right)$

$$
m\left(T_{n}\right)=\bar{m}_{x_{n-1}}+v^{(n-1)}\left(T_{n}\right)=\bar{m}_{x_{n}}+v^{(n)}\left(T_{n}\right), \quad\left\langle\bar{m}_{x_{n}}^{\prime}, v^{(n)}\left(T_{n}\right)\right\rangle=0 .
$$

Let

$$
h^{(n)}(t)=h(t), \quad t \in\left[T_{n}, T_{n+1}\right],
$$

introduce the stopping time w.r.t. the discrete filtration $\mathcal{F}_{T_{n+1}}$

$$
N_{\delta}:=\inf \left\{k:\left\|v^{(k)}\left(T_{k+1}\right)\right\|_{\infty}>\delta\right\}
$$

( $\delta$ as in Proposition 2.1) and define $g_{t}^{(n)}:=\exp \left\{t L_{x_{n}}\right\}, t \in \mathbb{R}_{+}$, with $L_{x_{n}}$ as in (2.3). We abbreviate $g_{t}=g_{t}^{(0)}$. 
After expanding $V^{\prime}(m(t))$ in (1.8) and (1.10) about $\bar{m}_{x_{n}}$, for $t \in\left[T_{n}, T_{n+1}\right]$ as long as $n \leq N_{\delta}, v^{(n)}(t)$ and $h^{(n)}(t)$ are given as the solution of

$$
\begin{aligned}
d v^{(n)}(t) & =\left[L_{x_{n}} v^{(n)}(t)+\lambda h^{(n)}(t)-3 \bar{m}_{x_{n}} v^{(n)}(t)^{2}-v^{(n)}(t)^{3}\right] d t+\lambda d W^{(a)}(t) \\
h^{(n)}(t) & =p_{t-T_{n}} h^{(n-1)}\left(T_{n}\right)-v^{(n)}(t)+p_{t-T_{n}} v^{(n)}\left(T_{n}\right)+\int_{T_{n}}^{t} d s \frac{\partial p_{t-s}}{\partial s} v^{(n)}(s)
\end{aligned}
$$

with initial condition $v^{(n)}\left(T_{n}\right)$ for the first equation. Recall that $v^{(n)}\left(T_{n}\right)$ is determined from $v^{(n-1)}\left(T_{n}\right)$ via $(3.2)$, and that from $(3.3)$ we have $h^{(n-1)}\left(T_{n}\right)=$ $h^{(n)}\left(T_{n}\right)$.

We will write in the next section $v^{(n)}(t)$ as a sum of 10 terms, $v^{(n)}(t)=$ $\sum_{i=1}^{10} \Gamma_{i}^{(n)}(t)$, but for the moment it is more convenient to keep some of the terms together. We are going to prove that for $n<N_{\delta}$,

$v^{(n)}(t)=\Gamma_{1}^{(n)}(t)+\Gamma_{2}^{(n)}(t)+\Lambda_{3}^{(n)}(t)+\Gamma_{4}^{(n)}(t)+\Gamma_{5}^{(n)}(t)+\Gamma_{6}^{(n)}(t)+\Gamma_{9}^{(n)}(t)+\Gamma_{10}^{(n)}(t)$

where

$$
\begin{aligned}
& \Gamma_{1}^{(n)}(t):=g_{t-T_{n}}^{(n)} v^{(n)}\left(T_{n}\right) \\
& \Gamma_{2}^{(n)}(t):=\lambda z^{(n)}(t):=\lambda \int_{T_{n}}^{t} g_{t-s}^{(n)} d W^{(a)}(s) \\
& \Lambda_{3}^{(n)}(t):=-\lambda \sum_{k=1}^{n} \int_{T_{n}}^{t} d s g_{t-s}^{(n)} p_{s-T_{k}}\left[\bar{m}_{x_{k}}-\bar{m}_{x_{k-1}}\right] \\
& \Gamma_{4}^{(n)}(t):=\lambda \int_{T_{n}}^{t} d s g_{t-s}^{(n)} \int_{T_{n}}^{s} d s^{\prime} \frac{\partial p_{s-s^{\prime}}}{\partial s^{\prime}} v^{(n)}\left(s^{\prime}\right) \\
& \Gamma_{5}^{(n)}(t):=-\lambda \int_{T_{n}}^{t} d s g_{t-s}^{(n)} v^{(n)}(s) \\
& \Gamma_{6}^{(n)}(t):=-\lambda \sum_{k=1}^{n} \int_{T_{n}}^{t} d s g_{t-s}^{(n)} \int_{T_{k-1}}^{T_{k}} d s^{\prime} \frac{\partial p_{s-s^{\prime}}}{\partial s} v^{(k-1)}\left(s^{\prime}\right) \\
& \Gamma_{9}^{(n)}(t):=-3 \int_{T_{n}}^{t} d s g_{t-s}^{(n)}\left[\bar{m}_{x_{n}} v^{(n)}(s)^{2}\right] \\
& \Gamma_{10}^{(n)}(t):=-\int_{T_{n}}^{t} d s g_{t-s}^{(n)}\left[v^{(n)}(s)^{3}\right] .
\end{aligned}
$$

Proof of (3.7). By applying $\left(\partial_{t}-L_{x_{n}}\right)^{-1}$ to $(3.5)$ we get

$$
v^{(n)}(t)=\Gamma_{1}^{(n)}(t)+\Gamma_{2}^{(n)}(t)+\Gamma_{9}^{(n)}(t)+\Gamma_{10}^{(n)}(t)+\lambda \int_{T_{n}}^{t} d s g_{t-s}^{(n)} h^{(n)}(s) \text {. }
$$


We use for $h^{(n)}(s)$ the expression given by (3.6), and get

$$
\begin{aligned}
v^{(n)}(t)= & \Gamma_{1}^{(n)}(t)+\Gamma_{2}^{(n)}(t)+\Gamma_{4}^{(n)}(t)+\Gamma_{5}^{(n)}(t)+\Gamma_{9}^{(n)}(t)+\Gamma_{10}^{(n)}(t) \\
& +\lambda \int_{T_{n}}^{t} d s g_{t-s}^{(n)} p_{s-T_{n}}\left[h^{(n-1)}\left(T_{n}\right)+v^{(n)}\left(T_{n}\right)\right] .
\end{aligned}
$$

To complete the proof of (3.7) we thus need to show

$$
\lambda \int_{T_{n}}^{t} d s g_{t-s}^{(n)} p_{s-T_{n}}\left[h^{(n-1)}\left(T_{n}\right)+v^{(n)}\left(T_{n}\right)\right]=\Lambda_{3}^{(n)}(t)+\Gamma_{6}^{(n)}(t) .
$$

From (3.2), we have

$$
h^{(n-1)}\left(T_{n}\right)+v^{(n)}\left(T_{n}\right)=h^{(n-1)}\left(T_{n}\right)+v^{(n-1)}\left(T_{n}\right)-\left(\bar{m}_{x_{n}}-\bar{m}_{x_{n-1}}\right)
$$

We use this identity in the l.h.s. of (3.16). The difference $\left(\bar{m}_{x_{n}}-\bar{m}_{x_{n-1}}\right)$ reproduces the last term of the sum in (3.10) (i.e. in the definition of $\left.\Lambda_{3}^{(n)}(t)\right)$. For the term with $h^{(n-1)}\left(T_{n}\right)+v^{(n-1)}\left(T_{n}\right)$, we use (3.6) to write

$$
\begin{aligned}
& h^{(n-1)}\left(T_{n}\right)+v^{(n-1)}\left(T_{n}\right) \\
& \quad=p_{T_{n}-T_{n-1}}\left\{h^{(n-1)}\left(T_{n-1}\right)+v^{(n-1)}\left(T_{n-1}\right)\right\}+\int_{T_{n-1}}^{T_{n}} d s^{\prime} \frac{\partial p_{T_{n}-s^{\prime}}}{\partial s^{\prime}} v^{(n-1)}\left(s^{\prime}\right) .
\end{aligned}
$$

The contribution of the last integral to the l.h.s. of (3.16) gives the last term of the sum in (3.13), i.e. the definition of $\Gamma_{6}^{(n)}(t)$. By iteration we then get (3.16) and (3.7) is therefore proved.

\section{A priori bounds}

We will use the representation (3.7) to prove in Proposition 4.1 below some a priori bounds on $v^{(n)}(t)$ and other quantities. We need first some more notation; recalling Proposition 2.1, we set

$$
\xi_{n}:=-\frac{3}{4} \sum_{k=0}^{n-1}\left\langle\bar{m}_{x_{k}}^{\prime}, v^{(k)}\left(T_{k+1}\right)\right\rangle
$$

We should think of $\xi_{n}$ as a linear approximation to $x_{n}$, since the increment $\xi_{k}-\xi_{k-1}$ is, according to $(2.5)$, the linear approximation to the displacement $x_{k}-x_{k-1}$ of the center in the time interval $\left[T_{k-1}, T_{k}\right]$.

We set

$$
V_{n}:=\sup _{t \in\left[T_{n}, T_{n+1}\right]}\left\|v^{(n)}(t)\right\|_{\infty}, \quad V_{n, *}:=\sup _{k \leq n} V_{k}, \quad V_{*}(\tau):=V_{n_{\lambda}(\tau), *}
$$


and

$$
\delta^{*}(\tau):=\sup _{k \leq n_{\lambda}(\tau)}\left|x_{k+1}-x_{k}\right|
$$

Let also

$$
Z_{n}:=\sup _{s \in\left[T_{n}, T_{n+1}\right]}\left\|z^{(n)}(s)\right\|_{\infty}, \quad Z_{n, *}:=\sup _{k \leq n} Z_{k}
$$

and $\mathcal{B}_{\lambda, \tau}^{(1)} \subset \Omega$ be the event (recall the definition of $\left(\Omega, \mathcal{F}, \mathcal{F}_{t}, \mathbb{P}\right)$ given in Section 1)

$$
\mathcal{B}_{\lambda, \tau}^{(1)}:=\left\{\omega \in \Omega: Z_{n_{\lambda}(\tau), *} \leq \lambda^{-\zeta} \sqrt{T}\right\} .
$$

We will prove in Appendix B the following Gaussian estimate: for each $\tau, \zeta, q>0$ there is a constant $C=C(\tau, \zeta, q)$ such that for any $\lambda>0$

$$
\mathbb{P}\left(\mathcal{B}_{\lambda, \tau}^{(1)}\right) \geq 1-C \lambda^{q}
$$

The next proposition is the key ingredient for the bound (1.11), see the beginning of $\S 8$ for the conclusion of the proof.

Proposition 4.1 For each $\tau, \zeta>0$ there is a constant $C=C(\tau, \zeta)$ such that, for any $\lambda>0$,

$$
V_{*}(\tau) \leq C \lambda^{1-\zeta} \sqrt{T}, \quad \delta^{*}(\tau) \leq C \lambda^{1-\zeta} \sqrt{T} \quad \text { on the set } \mathcal{B}_{\lambda, \tau}^{(1)} .
$$

In particular, for $\lambda>0$ small enough,

$$
N_{\delta}>n_{\lambda}(\tau) \quad \text { on the set } \mathcal{B}_{\lambda, \tau}^{(1)} .
$$

Proof. Let $\left(m_{\lambda}, t_{\lambda}\right), t_{\lambda} \in\left[T_{m_{\lambda}}, T_{m_{\lambda}+1}\right], m_{\lambda} \leq n_{\lambda}(\tau)$, be the stopping times $\left(m_{\lambda}\right.$ w.r.t. $\mathcal{F}_{T_{n+1}}, t_{\lambda}$ w.r.t. $\left.\mathcal{F}_{t}\right)$ so that $\left(m_{\lambda}, t_{\lambda}\right)=\left(n_{\lambda}(\tau), T_{n_{\lambda}(\tau)+1}\right)$ if $V_{*}(\tau)<\lambda T$, otherwise $\left(m_{\lambda}, t_{\lambda}\right)=(n, t)$, where $n$ is the first index such that $V_{n} \geq \lambda T$ and $t$ the first time in $\left[T_{n}, T_{n+1}\right]$ for which $\left\|v^{(n)}(t)\right\|_{\infty} \geq \lambda T$.

In the following we may assume $\lambda$ is small enough, otherwise (4.7) holds trivially. We claim there is a constant $C$ so that

$$
\left\|v^{\left(m_{\lambda}\right)}\left(t_{\lambda}\right)\right\|_{\infty} \leq C \lambda T
$$

In fact, if $t_{\lambda} \in\left(T_{m_{\lambda}}, T_{m_{\lambda}+1}\right]$, (4.9) follows from the continuity of $\left\|v^{\left(m_{\lambda}\right)}(t)\right\|_{\infty}$; otherwise, i.e. if $t_{\lambda}=T_{m_{\lambda}}$,

$$
\begin{aligned}
\left\|v^{\left(m_{\lambda}\right)}\left(T_{m_{\lambda}}\right)\right\|_{\infty} \leq\left\|v^{\left(m_{\lambda}-1\right)}\left(T_{m_{\lambda}}\right)\right\|_{\infty}+\| \bar{m}_{m_{\lambda}} & -\bar{m}_{m_{\lambda}-1} \| \\
& \leq \lambda T+\left|x_{m_{\lambda}}-x_{m_{\lambda}-1}\right| \leq C \lambda T
\end{aligned}
$$

from (3.2) and (2.4), since $\left\|\bar{m}^{\prime}\right\|_{\infty}=1$ and $\left\|v^{\left(m_{\lambda}-1\right)}\left(T_{m_{\lambda}}\right)\right\|_{\infty} \leq \lambda T$. 
We will study the evolution till time $t_{\lambda}$, so that our next considerations are tacitly restricted to $n \leq m_{\lambda}$ and $t \leq t_{\lambda}$.

By (4.9), $N_{\delta}>m_{\lambda}$ (for $\lambda$ small enough) so that we can use (3.7), regarding it as an integral equation in $v^{(n)}(t)$. We are going to bound one after the other all eight terms on the r.h.s. of (3.7).

We will use in the sequel the following bounds (see e.g. [9] and references therein). There is $C<\infty$ so that, for any measurable function $\varphi$,

$$
\left\|g_{t} \varphi\right\|_{\infty} \leq C\|\varphi\|_{\infty} .
$$

Moreover, by the Perron-Frobenius Theorem, there are $\alpha>0$ and $C<\infty$ so that for any $\varphi$ orthogonal to $\bar{m}^{\prime},\left\langle\bar{m}^{\prime}, \varphi\right\rangle=0$,

$$
\left\|g_{t} \varphi\right\|_{\infty} \leq C e^{-\alpha t}\|\varphi\|_{\infty} .
$$

Then, by the last identity in (3.2), for $t \in\left[T_{n}, T_{n+1}\right]$

$$
\begin{aligned}
\left\|\Gamma_{1}^{(n)}(t)\right\|_{\infty} & \leq C e^{-\alpha\left(t-T_{n}\right)}\left\|v^{(n)}\left(T_{n}\right)\right\|_{\infty} \\
& \leq C e^{-\alpha\left(t-T_{n}\right)}\left(\left\|v^{(n-1)}\left(T_{n}\right)\right\|_{\infty}+\left\|\bar{m}_{x_{n}}-\bar{m}_{x_{n-1}}\right\|_{\infty}\right) \\
& \leq C e^{-\alpha\left(t-T_{n}\right)}\left\|v^{(n-1)}\left(T_{n}\right)\right\|_{\infty} .
\end{aligned}
$$

The second inequality follows from (3.2) and the last one follows from (2.4), proceeding as in the proof of (4.9).

By definition (4.4) we get

$$
\sup _{t \in\left[T_{n}, T_{n+1}\right]}\left\|\Gamma_{2}^{(n)}(t)\right\|_{\infty} \leq C \lambda Z_{n} .
$$

We next bound $\Lambda_{3}^{(n)}$. We have

$$
\left\|p_{t} \bar{m}_{z}^{\prime}\right\|_{\infty} \leq \frac{2}{\sqrt{2 \pi t}} \leq \frac{1}{\sqrt{t}}
$$

because $p_{t}(x, y) \leq 1 / \sqrt{2 \pi t}$ and $\left\|\bar{m}_{z}^{\prime}\right\|_{1}=2$ for any $z \in \mathbb{R}$. Then

$$
\left\|p_{t}\left(\bar{m}_{x_{k}}-\bar{m}_{x_{k-1}}\right)\right\|_{\infty} \leq\left|\int_{x_{k-1}}^{x_{k}} d z\left\|p_{t} \bar{m}_{z}^{\prime}\right\|_{\infty}\right| \leq \frac{\left|x_{k}-x_{k-1}\right|}{\sqrt{t}}
$$

so that, by (4.10), we get

$$
\left\|\Lambda_{3}^{(n)}(t)\right\|_{\infty} \leq C \lambda \sum_{k=1}^{n} \int_{T_{n}}^{t} d s \frac{\left|x_{k}-x_{k-1}\right|}{\sqrt{s-T_{k}}} .
$$


By (2.4), we then get

$$
\left\|\Lambda_{3}^{(n)}(t)\right\|_{\infty} \leq C \lambda \sqrt{T} \sum_{k=1}^{n} \frac{V_{k-1}}{\sqrt{n-k+1}}
$$

We will next bound $\Gamma_{4}^{(n)}(t)$. We write

$$
g_{t-s}^{(n)}=p_{t-s}-\int_{s}^{t} d s^{\prime \prime} \frac{d}{d s^{\prime \prime}}\left(g_{t-s^{\prime \prime}}^{(n)} p_{s^{\prime \prime}-s}\right)=p_{t-s}+\int_{s}^{t} d s^{\prime \prime} g_{t-s^{\prime \prime}}^{(n)}\left[1-3 \bar{m}_{x_{n}}^{2}\right] p_{s^{\prime \prime}-s}
$$

having used (2.3) in the second equality. Then

$$
\begin{aligned}
\Gamma_{4}^{(n)}(t)= & \lambda \int_{T_{n}}^{t} d s \int_{T_{n}}^{s} d s^{\prime} \frac{\partial p_{t-s^{\prime}}}{\partial s^{\prime}} v^{(n)}\left(s^{\prime}\right) \\
& +\lambda \int_{T_{n}}^{t} d s \int_{s}^{t} d s^{\prime \prime} g_{t-s^{\prime \prime}}^{(n)}\left[1-3 \bar{m}_{x_{n}}^{2}\right] \int_{T_{n}}^{s} d s^{\prime} \frac{\partial p_{s^{\prime \prime}-s^{\prime}}}{\partial s^{\prime}} v^{(n)}\left(s^{\prime}\right)
\end{aligned}
$$

By using (4.10) and (see Appendix A)

$$
\left\|\frac{\partial}{\partial t} p_{t} \varphi\right\|_{\infty} \leq \frac{1}{t}\|\varphi\|_{\infty}
$$

since $\left\|v^{(n)}\left(s^{\prime}\right)\right\|_{\infty} \leq V_{n}$, we get

$$
\left\|\Gamma_{4}^{(n)}(t)\right\|_{\infty} \leq C \lambda T^{2} V_{n}
$$

This is not optimal but good enough for our purposes. Analogously

$$
\begin{gathered}
\left\|\Gamma_{5}^{(n)}(t)\right\|_{\infty} \leq C \lambda \int_{T_{n}}^{t} d s\left\|v^{(n)}(s)\right\|_{\infty} \leq C \lambda T V_{n} \\
\left\|\Gamma_{6}^{(n)}(t)\right\|_{\infty} \leq C \lambda \sum_{k=1}^{n} \int_{T_{n}}^{t} d s \int_{T_{k-1}}^{T_{k}} d s^{\prime} \frac{\left\|v^{(k-1)}\left(s^{\prime}\right)\right\|_{\infty}}{s-s^{\prime}} \leq C \lambda T \sum_{k=1}^{n} \frac{V_{k-1}}{n-k+1} .
\end{gathered}
$$

Finally, using again (4.10), we have

$$
\begin{aligned}
& \left\|\Gamma_{9}^{(n)}(t)\right\|_{\infty} \leq C T V_{n}^{2} \\
& \left\|\Gamma_{10}^{(n)}(t)\right\|_{\infty} \leq C T V_{n}^{3}
\end{aligned}
$$

hence, recalling the definition of $\left(m_{\lambda}, t_{\lambda}\right)$ and (4.9),

$$
\left\|\Gamma_{9}^{(n)}(t)\right\|_{\infty}+\left\|\Gamma_{10}^{(n)}(t)\right\|_{\infty} \leq C \lambda T^{2} V_{n}
$$


After collecting all these bounds, we have, for $t \in\left[T_{n}, T_{n+1}\right], n \leq m_{\lambda}, t \leq t_{\lambda}$,

$$
\begin{aligned}
\left\|v^{(n)}(t)\right\|_{\infty} \leq & C\left\{e^{-\alpha\left(t-T_{n}\right)}\left\|v^{(n-1)}\left(T_{n}\right)\right\|_{\infty}+\lambda Z_{n}+\lambda T^{2} V_{n}\right. \\
& \left.+\lambda \sqrt{T} \sum_{k=1}^{n} \frac{V_{k-1}}{\sqrt{n-k+1}}+\lambda T \sum_{k=1}^{n} \frac{V_{k-1}}{n-k+1}\right\} .
\end{aligned}
$$

By iterating once the above inequality we get

$$
\begin{aligned}
V_{n} \leq & C\left\{e^{-\alpha T} V_{n-2}+\lambda\left(Z_{n}+Z_{n-1}\right)+\lambda T^{2}\left(V_{n}+V_{n-1}\right)\right. \\
& \left.+\lambda \sqrt{T} \sum_{k=1}^{n} \frac{V_{k-1}}{\sqrt{n-k+1}}+\lambda T \sum_{k=1}^{n} \frac{V_{k-1}}{n-k+1}\right\} .
\end{aligned}
$$

Recalling (4.4), we have

$$
\begin{aligned}
V_{n} \leq & C\left\{e^{-\alpha T} V_{n, *}+\lambda Z_{n, *}+\lambda T^{2} V_{n, *}\right. \\
& \left.+\lambda \sqrt{T} \sum_{k=1}^{n} \frac{V_{k-1, *}}{\sqrt{n-k+1}}+\lambda T \sum_{k=1}^{n} \frac{V_{k-1, *}}{n-k+1}\right\}
\end{aligned}
$$

and since the r.h.s. is an increasing function of $n$,

$$
\begin{aligned}
V_{n, *} \leq & C\left\{e^{-\alpha T} V_{n, *}+\lambda Z_{n, *}+\lambda T^{2} V_{n, *}\right. \\
& \left.+\lambda \sqrt{T} \sum_{k=1}^{n} \frac{V_{k-1, *}}{\sqrt{n-k+1}}+\lambda T \sum_{k=1}^{n} \frac{V_{k-1, *}}{n-k+1}\right\} .
\end{aligned}
$$

Inequality (4.25) yields, for $n \leq m_{\lambda} \leq n_{\lambda}(\tau)$,

$$
\left[1-C\left(\lambda T^{2}+e^{-\alpha T}+\lambda T \log n_{\lambda}(\tau)\right)\right] V_{n, *} \leq C \lambda Z_{n, *}+\sum_{k=0}^{n-1} \frac{C \lambda \sqrt{T}}{\sqrt{n-k}} V_{k, *} .
$$

For $\lambda$ small enough the square bracket term is larger than $1 / 2$, so that (provided we double the value of the constant $C$ )

$$
V_{n, *} \leq C \lambda Z_{n, *}+\sum_{k=0}^{n-1} \frac{C \lambda \sqrt{T}}{\sqrt{n-k}} V_{k, *} .
$$

By iteration of (4.26) we get

$$
V_{n, *} \leq C \lambda Z_{n, *}+\sum_{k=0}^{n-1} \frac{C^{2} \lambda^{2} \sqrt{T}}{\sqrt{n-k}} Z_{k, *}+C^{2} \lambda^{2} T \sum_{k=0}^{n-2} \alpha(n, k) V_{k, *}
$$


where

$$
\alpha(n, k)=\sum_{h=k+1}^{n-1} \frac{1}{\sqrt{n-h}} \frac{1}{\sqrt{h-k}}
$$

is a bounded function of $n$ and $k$.

We bound the second term on the r.h.s. of (4.27) by

$$
Z_{n, *} \sum_{k=0}^{n-1} \frac{C^{2} \lambda^{2} \sqrt{T}}{\sqrt{n-k}} \leq C Z_{n, *} \lambda^{2} \sqrt{T n} \leq C \lambda Z_{n, *}
$$

since $n \leq m_{\lambda} \leq\left(\lambda^{2} T\right)^{-1} \tau$. We then get from (4.27)

$$
V_{n, *} \leq C \lambda Z_{n, *}+C \lambda^{2} T \sum_{k=0}^{n-2} V_{k, *}
$$

from which, by Gronwall Lemma, there is $C=C(\tau)$ such that

$$
V_{n, *} \leq C \lambda Z_{n, *}, \quad \text { for all } n \leq m_{\lambda} .
$$

By (4.5), choosing $\lambda$ small enough, we have, for $n \leq m_{\lambda}$,

$$
V_{n, *} \leq C \lambda^{1-\zeta} \sqrt{T} \quad \text { on the set } \mathcal{B}_{\lambda, \tau}^{(1)}
$$

which implies $m_{\lambda}=n_{\lambda}(\tau)$ on the set $\mathcal{B}_{\lambda, \tau}^{(1)}$. Thus the first bound in (4.7) follows from (4.5) and (4.30). Since $N_{\delta}>m_{\lambda}$, (4.8) also follows. Since $\left\|\bar{m}^{\prime}\right\|_{\infty}=1$, the second estimate in (4.7) follows directly from the first one and Proposition 2.1.

We are going to prove that the component of $v^{(n)}\left(T_{n+1}\right)$ orthogonal to $\bar{m}_{x_{n}}^{\prime}$, is bounded by $C \lambda^{1-\zeta}$, thus considerably improving the bound on the full $v^{(n)}\left(T_{n+1}\right)$. Let

$$
g_{t}^{(n, \perp)}:=g_{t}^{(n)}\left(1-\frac{3}{4}\left|\bar{m}_{x_{n}}^{\prime}\right\rangle\left\langle\bar{m}_{x_{n}}^{\prime}\right|\right)
$$

the operator whose kernel is

$$
g_{t}^{(n, \perp)}(x, y)=g_{t}^{(n)}(x, y)-\frac{3}{4} \bar{m}_{x_{n}}^{\prime}(x) \bar{m}_{x_{n}}^{\prime}(y)
$$

The superscript $\perp$ recalls $L_{2}$-orthogonality w.r.t. the eigenvector $\bar{m}_{x_{n}}^{\prime}$ of $g_{t}^{(n)}$, i.e. $g_{t}^{(n)} \bar{m}_{x_{n}}^{\prime}=\bar{m}_{x_{n}}^{\prime}$. It follows from (4.11) that there are constants $\alpha>0$ and $C<\infty$ so that, for any $\varphi$,

$$
\left\|g_{t}^{(n, \perp)} \varphi\right\|_{\infty} \leq C e^{-\alpha t}\|\varphi\|_{\infty} .
$$

Let also

$$
z^{(n, \perp)}(t):=z^{(n)}(t)-\frac{3}{4}\left\langle\bar{m}_{x_{n}}^{\prime}, z^{(n)}(t)\right\rangle \bar{m}_{x_{n}}^{\prime}
$$


be the component of $z^{(n)}(t)$ orthogonal to $\bar{m}_{x_{n}}^{\prime}$ and introduce the event

$$
\mathcal{B}_{\lambda, \tau}^{(2)}:=\left\{\omega \in \Omega: \sup _{n \leq n_{\lambda}(\tau)} \sup _{t \in\left[T_{n}, T_{n+1}\right]}\left\|z^{(n, \perp)}(t)\right\|_{\infty} \leq \lambda^{-\zeta}\right\} .
$$

In Appendix B we will prove that for each $\tau, \zeta, q>0$ there exists a constant $C=C(\tau, \zeta, q)$ such that for any $\lambda>0$

$$
\mathbb{P}\left(\mathcal{B}_{\lambda, \tau}^{(2)}\right) \geq 1-C \lambda^{q}
$$

Define now

$$
\begin{gathered}
V_{*}^{\perp}(\tau):=\sup _{n \leq n_{\lambda}(\tau)}\left\|v^{(n, \perp)}\left(T_{n+1}\right)\right\|_{\infty}, \\
v^{(n, \perp)}\left(T_{n+1}\right):=v^{(n)}\left(T_{n+1}\right)-\frac{3}{4}\left\langle\bar{m}_{x_{n}}^{\prime}, v^{(n)}\left(T_{n+1}\right)\right\rangle \bar{m}_{x_{n}}^{\prime} .
\end{gathered}
$$

Proposition 4.2 Recalling (4.5) and (4.34), set

$$
\mathcal{B}_{\lambda, \tau}^{(1,2)}:=\mathcal{B}_{\lambda, \tau}^{(1)} \cap \mathcal{B}_{\lambda, \tau}^{(2)} .
$$

Then, for each $\tau, \zeta>0$ there is a constant $C=C(\tau, \zeta)$ such that, for any $\lambda>0$,

$$
V_{*}^{\perp}(\tau) \leq C \lambda^{1-2 \zeta} \quad \text { on the set } \mathcal{B}_{\lambda, \tau}^{(1,2)}
$$

and, recalling (4.1) for the definition of $\xi_{n}$,

$$
\sup _{n \leq n_{\lambda}(\tau)+1}\left|\xi_{n}-x_{n}\right| \leq C T^{-1 / 2+\zeta} \quad \text { on the set } \mathcal{B}_{\lambda, \tau}^{(1,2)} .
$$

Proof. Let

$$
\begin{aligned}
\Lambda_{3}^{(n, \perp)}\left(T_{n+1}\right) & :=\Lambda_{3}^{(n)}\left(T_{n+1}\right)-\frac{3}{4}\left\langle\bar{m}_{x_{n}}^{\prime}, \Lambda_{3}^{(n)}\left(T_{n+1}\right)\right\rangle \bar{m}_{x_{n}}^{\prime} \\
& =-\lambda \sum_{k=1}^{n} \int_{T_{n}}^{T_{n+1}} d t g_{T_{n+1}-t}^{(n, \perp)} p_{t-T_{k}}\left[\bar{m}_{x_{k}}-\bar{m}_{x_{k-1}}\right]
\end{aligned}
$$

where we used (4.31). By using (4.14), (4.32), $\left\|\bar{m}^{\prime}\right\|_{\infty} \leq 1$, and recalling (4.3) we have

$$
\begin{aligned}
\left\|\Lambda_{3}^{(n, \perp)}\left(T_{n+1}\right)\right\|_{\infty} & \leq C \delta^{*}(\tau) \lambda \sum_{k=1}^{n} \int_{T_{n}}^{T_{n+1}} d t e^{-\alpha\left(T_{n+1}-t\right)} \frac{1}{\sqrt{t-T_{k}}} \\
& \leq C \delta^{*}(\tau) \frac{\lambda}{\sqrt{T}} \sqrt{n_{\lambda}(\tau)} \leq C \lambda^{1-\zeta} T^{-1 / 2}
\end{aligned}
$$

the last inequality being true, by (4.7), on the set $\mathcal{B}_{\lambda, \tau}^{(1)}$. 
Shorthanding by $R^{(n)}$ the sum of all the terms on the r.h.s. of (3.7), except for $\Gamma_{2}^{(n)}$ and $\Lambda_{3}^{(n)}$, and calling $R^{(n, \perp)}$ its orthogonal projection,

$$
v^{(n, \perp)}\left(T_{n+1}\right)=\lambda z^{(n, \perp)}\left(T_{n+1}\right)+\Lambda_{3}^{(n, \perp)}\left(T_{n+1}\right)+R^{(n, \perp)} .
$$

The last term is bounded by

$$
\left|R^{(n, \perp)}\right| \leq C\left[e^{-\alpha T}+\lambda T^{2}+\lambda T \log n_{\lambda}(\tau)\right] V_{*}(\tau)
$$

as it follows from (4.12) and (4.18)-(4.20), and (4.23). The bound (4.38) now follows from the definition (4.34), and equations (4.41)-(4.43).

It remains to prove (4.39). Recalling that $x_{0}=0$, from (2.5) and (4.1),

$$
\begin{array}{r}
x_{n}=\sum_{k=0}^{n-1}\left(x_{k+1}-x_{k}\right)=\xi_{n}-\frac{9}{16} \sum_{k=0}^{n-1}\left\langle\bar{m}_{x_{k}}^{\prime}, v^{(k)}\left(T_{k+1}\right)\right\rangle\left\langle\bar{m}_{x_{k}}^{\prime \prime}, v^{(k)}\left(T_{k+1}\right)\right\rangle \\
+\sum_{k=0}^{n-1} R\left(v^{(k)}\left(T_{k+1}\right)\right)
\end{array}
$$

then, for $n \leq n_{\lambda}(\tau)$, since $\left\|\bar{m}^{\prime}\right\|_{1}=2$,

$$
\begin{array}{r}
\left|\xi_{n}-x_{n}\right| \leq n_{\lambda}(\tau)\left(2 V_{*}(\tau) \sup _{n \leq n_{\lambda}(\tau)}\left|\left\langle\bar{m}_{x_{n}}^{\prime \prime}, v^{(n)}\left(T_{n+1}\right)\right\rangle\right|+\right. \\
\left.\sup _{\substack{n \leq n_{\lambda}(\tau)}}\left\|R\left(v^{(n)}\left(T_{n+1}\right)\right)\right\|_{\infty}\right) .
\end{array}
$$

By (2.5)

$$
\sup _{n \leq n_{\lambda}(\tau)}\left\|R\left(v^{(n)}\left(T_{n+1}\right)\right)\right\|_{\infty} \leq C V_{*}(\tau)^{3} .
$$

Since $\left\langle\bar{m}^{\prime \prime}, \bar{m}^{\prime}\right\rangle=0,\left|\left\langle\bar{m}_{x_{n}}^{\prime \prime}, v^{(n)}\left(T_{n+1}\right)\right\rangle\right| \leq C V_{*}^{\perp}(\tau)$, (4.39) follows from (4.7), (4.38), (4.45), and (4.46).

The bound in (4.38) holds also for $v^{(n)}\left(T_{n}\right)$. Indeed, since $\left\langle\bar{m}_{x_{n}}^{\prime}, v^{(n)}\left(T_{n}\right)\right\rangle=0$, we have the following proposition.

Proposition 4.3 Let $\mathcal{B}_{\lambda, \tau}^{(1,2)}$ be as in (4.37). Then, for each $\tau, \zeta>0$ there is a constant $C=C(\tau, \zeta)$ such that, for any $\lambda>0$

$$
\sup _{n \leq n_{\lambda}(\tau)}\left\|v^{(n)}\left(T_{n}\right)\right\|_{\infty} \leq C \lambda^{1-2 \zeta} \quad \text { on the set } \mathcal{B}_{\lambda, \tau}^{(1,2)}
$$

Proof. By (3.2)

$$
v^{(n)}\left(T_{n}\right)=v^{(n-1)}\left(T_{n}\right)+\bar{m}_{x_{n-1}}-\bar{m}_{x_{n}}
$$


By Taylor expansion up to fourth order, we have

$$
\begin{aligned}
\bar{m}_{x_{n}}-\bar{m}_{x_{n-1}}= & -\bar{m}_{x_{n-1}}^{\prime}\left(x_{n}-x_{n-1}\right)+\frac{1}{2} \bar{m}_{x_{n-1}}^{\prime \prime}\left(x_{n}-x_{n-1}\right)^{2} \\
& -\frac{1}{6} \bar{m}_{x_{n-1}}^{\prime \prime \prime}\left(x_{n}-x_{n-1}\right)^{3}+a_{n-1}\left(x_{n}-x_{n-1}\right)^{4}
\end{aligned}
$$

where $a_{n-1}$ is bounded. Thus, by Proposition 2.1, we have

$$
\bar{m}_{x_{n}}-\bar{m}_{x_{n-1}}=\frac{3}{4}\left\langle\bar{m}_{x_{n-1}}^{\prime}, v^{(n-1)}\left(T_{n}\right)\right\rangle \bar{m}_{x_{n-1}}^{\prime}+\alpha_{n}
$$

where

$$
\begin{aligned}
& \alpha_{n}=\frac{1}{2} \bar{m}_{x_{n-1}}^{\prime \prime}\left(x_{n}-x_{n-1}\right)^{2}-\frac{1}{6} \bar{m}_{x_{n-1}}^{\prime \prime \prime}\left(x_{n}-x_{n-1}\right)^{3}+a_{n-1}\left(x_{n}-x_{n-1}\right)^{4} \\
& +\bar{m}_{x_{n-1}}^{\prime}\left\{\frac{9}{16}\left\langle\bar{m}_{x_{n-1}}^{\prime \prime}, v^{(n-1)}\left(T_{n}\right)\right\rangle\left\langle\bar{m}_{x_{n-1}}^{\prime}, v^{(n-1)}\left(T_{n}\right)\right\rangle-R\left(v^{(n-1)}\left(T_{n}\right)\right)\right\} .
\end{aligned}
$$

From (4.48) and (4.50) we get

$$
v^{(n)}\left(T_{n}\right)=v^{(n-1, \perp)}\left(T_{n}\right)-\alpha_{n} .
$$

Note that, from (4.7), on the set $\mathcal{B}_{\lambda, \tau}^{(1,2)}$ we have $\sup _{n \leq n_{\lambda}(\tau)}\left|x_{n}-x_{n-1}\right| \leq C \lambda^{1-\zeta} \sqrt{T}$; by (4.38) and (4.46) we have $\left|\alpha_{n}\right| \leq C \lambda^{2-2 \zeta} T$ (a better bound is proved in Section $6)$. The bound (4.47) follows.

\section{Part III. Limit motion}

\section{A new integral equation}

Unless otherwise stated, we will work in the set $\mathcal{B}_{\lambda, \tau}^{(1,2)}$ which appears in Propositions 4.2 and 4.3. In particular we can use the integral representation (3.7) for all $n \leq n_{\lambda}(\tau)$ (if $\lambda$ is small enough) and the bounds of Section 4. It is now convenient to decompose the term $\Lambda_{3}^{(n)}(t)$ into the sum of three new terms; we thus use $(4.50)$ to write

$$
\Lambda_{3}^{(n)}(t)=\Gamma_{3+8}^{(n)}(t)+\Gamma_{7}^{(n)}(t)
$$

where

$$
\begin{aligned}
\Gamma_{3+8}^{(n)}(t) & :=-\frac{3 \lambda}{4} \sum_{k=1}^{n}\left\langle\bar{m}_{x_{k-1}}^{\prime}, v^{(k-1)}\left(T_{k}\right)\right\rangle \int_{T_{n}}^{t} d s g_{t-s}^{(n)} p_{s-T_{k}} \bar{m}_{x_{k-1}}^{\prime} \\
\Gamma_{7}^{(n)}(t) & :=-\lambda \sum_{k=1}^{n} \int_{T_{n}}^{t} d s g_{t-s}^{(n)} p_{s-T_{k}} \alpha_{k}
\end{aligned}
$$


and next we decompose $\Gamma_{3+8}^{(n)}(t)=\Gamma_{3}^{(n)}(t)+\Gamma_{8}^{(n)}(t)$ where

$$
\begin{gathered}
\Gamma_{3}^{(n)}(t):=-\frac{3 \lambda}{4} \sum_{k=0}^{n-1}\left\langle\bar{m}_{x_{k}}^{\prime}, v^{(k)}\left(T_{k+1}\right)\right\rangle \int_{T_{n}}^{t} d s g_{t-s}^{(n)} \frac{2}{\sqrt{2 \pi\left(s-T_{k+1}\right)}} \\
\Gamma_{8}^{(n)}(t):=-\frac{3 \lambda}{4} \sum_{k=0}^{n-1}\left\langle\bar{m}_{x_{k}}^{\prime}, v^{(k)}\left(T_{k+1}\right)\right\rangle \int_{T_{n}}^{t} d s g_{t-s}^{(n)}\left[p_{s-T_{k+1}} \bar{m}_{x_{k}}^{\prime}-\frac{2}{\sqrt{2 \pi\left(s-T_{k+1}\right)}}\right] .
\end{gathered}
$$

Note that $\Gamma_{3}^{(n)}(t)$ is obtained from $\Gamma_{3+8}^{(n)}(t)$ replacing $p_{t}(x, y)$ by $(2 \pi t)^{-1 / 2}$ (recall that $\int d x \bar{m}^{\prime}(x)=2$ ). In conclusion we have

$$
v^{(n)}(t)=\sum_{i=1}^{10} \Gamma_{i}^{(n)}(t)
$$

Let us define

$$
\psi_{n}:=-\frac{3}{4}\left\langle\bar{m}_{x_{n}}^{\prime}, v^{(n)}\left(T_{n+1}\right)\right\rangle
$$

so that, recalling (4.1),

$$
\xi_{n}=\sum_{k=0}^{n-1} \psi_{k}
$$

We then set $t=T_{n+1}$ in (5.6) and project it on $\bar{m}_{x_{n}}^{\prime}$, getting

$$
\psi_{n}=\eta_{n}-\sum_{k=0}^{n-1} A_{n, k} \psi_{k}
$$

where

$$
\eta_{n}:=\sum_{\substack{i=1 \\ i \neq 3}}^{10} \eta_{n}(i), \quad \eta_{n}(i)=-\frac{3}{4}\left\langle\bar{m}_{x_{n}}^{\prime}, \Gamma_{i}^{(n)}\left(T_{n+1}\right)\right\rangle, i=1, \ldots, 10
$$

and

$$
A_{n, k}:=\lambda \frac{3}{\sqrt{2 \pi}} \frac{2 \sqrt{T}}{\sqrt{n-k}+\sqrt{n-k-1}} \mathbf{1}_{k<n} .
$$

Note that

$$
\eta_{n}(3) \equiv-\sum_{k=0}^{n-1} A_{n, k} \psi_{k}
$$

Despite its appearance, (5.9) is not a linear equation in the variables $\psi_{n}$, because the term $\eta_{n}$ still depends on the unknowns $v^{(n)}(t)$. We shall however see, using the a priori bounds of Section 4 , that all contributions to $\eta_{n}$ which contain the unknowns vanish as $\lambda \downarrow 0$ (this is not exactly true, as some terms will vanish 
only after averaging over $n)$. As already seen in Section 4 the matrix $A:=\left(\left(A_{n, k}\right)\right)$ improves after iterations. Calling $\psi$ and $\eta$ the vectors whose components are $\psi_{n}$ and $\eta_{n},(5.9)$ becomes

$$
\psi=\eta-A \psi .
$$

In $\mathbb{R}^{n_{\lambda}(\tau)+1}$ we introduce the seminorms $|y|_{n}:=\sup _{0 \leq j \leq n}\left|y_{j}\right|$ and the corresponding seminorms on matrices as

$$
\|M\|_{n}:=\sup _{|y|_{n}=1}|M y|_{n}=\sup _{0 \leq i \leq n} \sum_{j=0}^{n}\left|M_{i, j}\right| .
$$

By the triangular structure of $A$, see (5.11), we easily get the following lemma.

Lemma 5.1 For each $\tau>0$ there is a constant $C=C(\tau)$ such that for any $\lambda>0$

$$
\sup _{n \leq n_{\lambda}(\tau)}\left\|A^{j}\right\|_{n} \leq \frac{C^{j}}{j !} .
$$

Hence, from (5.13),

$$
\psi=\sum_{j=0}^{\infty}(-A)^{j} \eta=(1+A)^{-1} \eta, \quad \sup _{n \leq n_{\lambda}(\tau)}\left\|(1+A)^{-1}\right\|_{n} \leq C .
$$

It will be convenient to consider also one iteration of (5.13), i.e.

$$
\psi=\eta-A \eta+A^{2} \psi
$$

Explicitly we have

$$
\left(A^{2}\right)_{n, k}=\sum_{j=k+1}^{n-1} A_{n, j} A_{j, k} \mathbf{1}_{0 \leq k<n-1}
$$

We conclude the section by showing that $A^{2}$ has a scaling limit.

Proposition 5.2 For each $\tau>0$ there is a constant $C=C(\tau)$ such that

$$
\sup _{0 \leq k<n \leq n_{\lambda}(\tau)}\left|\left(A^{2}\right)_{n, k}\right| \leq C \lambda^{2} T \text {. }
$$

Moreover, for each $\delta, \tau>0$,

$$
\lim _{\lambda \downarrow 0} \sup _{\substack{0 \leq \tau_{2}<\tau_{1} \leq \tau \\ \tau_{1}-\tau_{2}>\delta}}\left|\left(\lambda^{2} T\right)^{-1}\left(A^{2}\right)_{n_{\lambda}\left(\tau_{1}\right), n_{\lambda}\left(\tau_{2}\right)}-\frac{9}{2}\right|=0 .
$$


Proof. By (5.11)

$$
\left|\left(A^{2}\right)_{n, k}\right| \leq C \lambda^{2} T \sum_{j=k+1}^{n-1} \frac{1}{\sqrt{n-j} \sqrt{j-k}}
$$

which proves (5.19).

By (5.11) we have

$$
\begin{aligned}
\left(\lambda^{2} T\right)^{-1} & \left(A^{2}\right)_{n_{\lambda}\left(\tau_{1}\right), n_{\lambda}\left(\tau_{2}\right)-\frac{9}{2}=\frac{9}{2 \pi}} \\
& \times\left\{\sum_{j=n_{\lambda}\left(\tau_{2}\right)+1}^{n_{\lambda}\left(\tau_{1}\right)-1} \frac{2}{\sqrt{n_{\lambda}\left(\tau_{1}\right)-j}+\sqrt{n_{\lambda}\left(\tau_{1}\right)-j-1}}\right. \\
& \left.\times \frac{2}{\sqrt{j-n_{\lambda}\left(\tau_{2}\right)}+\sqrt{j-n_{\lambda}\left(\tau_{2}\right)-1}}-\pi\right\} .
\end{aligned}
$$

Since $\pi=\int_{0}^{1} d x[x(1-x)]^{-1 / 2}$, by letting $\sigma=\tau_{1}-\tau_{2}$ and changing the index of summation the r.h.s. of (5.21) equals

$$
\frac{9}{2 \pi}\left\{\sum_{j=0}^{n_{\lambda}(\sigma)-2} \frac{2}{\sqrt{n_{\lambda}(\sigma)-j}+\sqrt{n_{\lambda}(\sigma)-j-1}} \frac{2}{\sqrt{j}+\sqrt{j+1}}-\int_{0}^{1} d x \frac{1}{\sqrt{x(1-x)}}\right\}
$$

which converges, as $\lambda \downarrow 0$, to 0 uniformly for $\sigma \in[\delta, \tau]$, as can be easily checked. Proposition 5.2 is proved.

\section{Bounds on $\eta$}

Recalling (5.8)-(5.10), a term $\eta(i)$ (as in the previous section, we are here using vectorial notation) contributing to $\psi$ whose seminorm is $|\eta(i)|_{n}=o\left(\lambda^{2} T\right)$ (i.e. such that $\left(\lambda^{2} T\right)^{-1}|\eta(i)|_{n}$ vanishes as $\left.\lambda \downarrow 0\right)$ does not contribute to the limiting equation for $\xi_{n}$ since $n \leq\left(\lambda^{2} T\right)^{-1} \tau$. This is the case for some of the $\eta(i)$ 's, i.e. $\eta(1), \eta(7)$, and $\eta(10)$, as we shall see.

Clearly $\eta_{n}(2)$ is not negligible because its typical magnitude is $\lambda \sqrt{T}$. It will be examined in the next section, where we shall see that, summed over $n$ it gives a finite contribution because of cancellations related to its martingale nature.The other terms, i.e. $\eta_{n}(i)$, with $i$ from 4 to 9 , live on an intermediate stage: they are smaller than the a priori bound $\lambda^{1-\zeta} \sqrt{T}$, yet not small enough to be directly negligible. We shall rewrite the factors $v^{(n)}(t)$ via (5.6), with the idea that if we get two $\eta(i), i=4, \ldots, 10$ then the corresponding terms become negligible. We will use the following notation.

Definition 6.1 Let $\eta_{n}\left(i_{1}, \ldots, i_{k}\right), k>1, i_{j} \in\{3,4,5,6,8\}$ when $j<k, i_{k} \in$ $\{1, \ldots, 10\}$, be the term which is obtained from $\eta_{n}\left(i_{1}\right)$ by replacing the $v^{(\ell)}(\cdot)-$ function in its expression by $\Gamma_{i_{2}}^{(\ell)}(\cdot)$; then, the new $v^{\left(\ell^{\prime}\right)}(\cdot)$ function which appears 
is replaced by $\Gamma_{i_{3}}^{\left(\ell^{\prime}\right)}(\cdot)$ and so forth till the last one, which is not changed. Instead of an index $i_{j}$ may appear the symbol $3+8$ or $4+5$, it means that, at that stage of the iteration, we replace the $v^{(\ell)}(\cdot)$-function by $\Gamma_{3}^{(\ell)}(\cdot)+\Gamma_{8}^{(\ell)}(\cdot)$, or respectively by $\Gamma_{4}^{(\ell)}(\cdot)+\Gamma_{5}^{(\ell)}(\cdot)$.

We will also consider $i_{1}=9$, in which case there is a product of two $v$ functions. Then, $\eta_{n}\left(9 \mid i_{2}, \ldots, i_{k} ; i_{2}^{\prime}, \ldots, i_{h}^{\prime}\right)$ is defined by doing the branching $i_{2}, \ldots, i_{k}$ (with the previous rules) for the first $v$ function and the branching $i_{2}^{\prime}, \ldots, i_{h}^{\prime}$ for the second one (the second branching may be absent).

We define

$$
\sigma_{n}:=\eta_{n}(2)+\eta_{n}(4+5,2)+\eta_{n}(6,2)+\eta_{n}(9 \mid 2 ; 2)
$$

which will be studied in the next section where we will bound its sum over $n$ using probabilistic arguments, $\sigma_{n}$ is in fact a truly stochastic term. The difference between $\eta_{n}$ and $\sigma_{n}$ is negligible, this being the main result in this section. Let

$$
\begin{aligned}
\mathcal{B}_{\lambda, \tau}^{(3)} & :=\left\{\omega \in \Omega: \sup _{n \leq n_{\lambda}(\tau)}\left|\sum_{k<n} A_{n, k} \eta_{k}(2)\right| \leq \lambda^{2-\zeta} T\right\} \\
\mathcal{B}_{\lambda, \tau}^{(4)} & :=\left\{\omega \in \Omega: \sup _{n \leq n_{\lambda}(\tau)}\left|\sum_{k<n} \frac{\lambda \sqrt{T}}{\sqrt{n-k}} \eta_{k}(2)\right| \leq \lambda^{2-\zeta} T\right\}
\end{aligned}
$$

where $\eta_{k}(2)$ and $A_{n, k}$ are defined in (5.10) and (5.11) respectively. We will prove in Appendix B that for each $\tau, \zeta, q>0$ there is $C=C(\tau, \zeta, q)$ so that

$$
\mathbb{P}\left(\mathcal{B}_{\lambda, \tau}^{(3)} \cap \mathcal{B}_{\lambda, \tau}^{(4)}\right) \geq 1-C \lambda^{q}
$$

Finally define

$$
x_{n, *}:=\sup _{k \leq n}\left|x_{n}\right|, \quad x_{*}(\tau)=x_{n_{\lambda}(\tau), *} .
$$

Proposition 6.2 Recalling (4.37), let

$$
\mathcal{B}_{\lambda, \tau}:=\mathcal{B}_{\lambda, \tau}^{(1,2)} \cap \mathcal{B}_{\lambda, \tau}^{(3)} \cap \mathcal{B}_{\lambda, \tau}^{(4)}
$$

Then for each $\tau>0$, there is a constant $C=C(\tau)$ so that for any $\lambda>0$ and $\zeta$ small enough

$$
\left|\eta_{n}-\sigma_{n}\right| \leq C \lambda^{2} T^{3 / 4}\left(1+x_{n+1, *}^{2}\right) \quad \text { on the set } \mathcal{B}_{\lambda, \tau} .
$$

Proof. We will call negligible a term which is bounded by the r.h.s. of (6.7). We will next examine one by one all the terms which contribute to $\eta_{n}-\sigma_{n}$ and show that they are all negligible, thus proving Proposition 6.2. 
Step 1. The terms $\eta_{n}(1)$ and $\eta_{n}(10)$. We have that $\eta_{n}(1)=0$; indeed by (3.8), since $g_{t}^{(n)}$ is self-adjoint and $g_{t}^{(n)} \bar{m}_{x_{n}}^{\prime}=\bar{m}_{x_{n}}^{\prime}$,

$$
\eta_{n}(1)=-\frac{3}{4}\left\langle\bar{m}_{x_{n}}^{\prime}, g_{T}^{(n)} v^{(n)}\left(T_{n}\right)\right\rangle=-\frac{3}{4}\left\langle\bar{m}_{x_{n}}^{\prime}, v^{(n)}\left(T_{n}\right)\right\rangle=0
$$

by $(3.2)$.

To show $\eta_{n}(10)$ is negligible we use the bound (4.22); since we are in the set $\mathcal{B}_{\lambda, \tau}$, we get, from (4.7)

$$
\sup _{t \in\left[T_{n}, T_{n+1}\right]}\left\|\Gamma_{10}^{(n)}(t)\right\|_{\infty} \leq C T^{5 / 2} \lambda^{3-3 \zeta} \quad \text { hence } \quad\left|\eta_{n}(10)\right| \leq C T^{5 / 2} \lambda^{3-3 \zeta} .
$$

Step 2. The term $\eta_{n}(7)$. By (5.3) and (4.51),

$$
\Gamma_{7}^{(n)}(t)=I_{1}+I_{2}+I_{3}+I_{4}
$$

where

$$
\begin{aligned}
I_{1} & :=-\frac{\lambda}{2} \sum_{k=1}^{n}\left(x_{k}-x_{k-1}\right)^{2} \int_{T_{n}}^{t} d s g_{t-s}^{(n)} p_{s-T_{k}} \bar{m}_{x_{k-1}}^{\prime \prime} \\
I_{2} & :=-\lambda \sum_{k=1}^{n}\left[\frac{\left(x_{k}-x_{k-1}\right)^{3}}{6} \int_{T_{n}}^{t} d s g_{t-s}^{(n)} p_{s-T_{k}} \bar{m}_{x_{k-1}}^{\prime \prime \prime}\right. \\
& \left.+\left(x_{k}-x_{k-1}\right)^{4} \int_{T_{n}}^{t} d s g_{t-s}^{(n)} p_{s-T_{k}} a_{k-1}\right] \\
I_{3} & :=-\frac{9 \lambda}{16} \sum_{k=1}^{n}\left\langle\bar{m}_{x_{k-1}^{\prime \prime}}^{\prime \prime} v^{(k-1)}\left(T_{k}\right)\right\rangle\left\langle\bar{m}_{x_{k-1}}^{\prime}, v^{(k-1)}\left(T_{k}\right)\right\rangle \int_{T_{n}}^{t} d s g_{t-s}^{(n)} p_{s-T_{k}} \bar{m}_{x_{k-1}}^{\prime} \\
I_{4} & :=\lambda \sum_{k=1}^{n} \int_{T_{n}}^{t} d s g_{t-s}^{(n)} p_{s-T_{k}}\left[\bar{m}_{x_{k-1}}^{\prime} R\left(v^{(k-1)}\left(T_{k}\right)\right)\right]
\end{aligned}
$$

To bound $I_{1}$ we first consider the term with $k=n$. We write, for $t>T_{n}+1$,

$$
\int_{T_{n}}^{t} d s g_{t-s}^{(n)} p_{s-T_{n}} \bar{m}_{x_{n-1}}^{\prime \prime}=\left\{\int_{T_{n}}^{T_{n}+1}+\int_{T_{n}+1}^{t}\right\} d s g_{t-s}^{(n)} p_{s-T_{n}} \bar{m}_{x_{n-1}}^{\prime \prime} .
$$

The first integral is bounded by a constant. In the second integral, as well as in the integrals in $I_{1}$ when $k<n$, we write

$$
p_{s-T_{k}} \bar{m}_{x_{k-1}}^{\prime \prime}=2 \frac{\partial p_{s-T_{k}}}{\partial s} \bar{m}_{x_{k-1}}
$$


and then use (4.17). We thus get recalling the definition (4.3) of $\delta^{*}(\tau)$, and using (4.10),

$$
\left\|I_{1}\right\|_{\infty} \leq C \lambda \delta^{*}(\tau)^{2}\left\{\log T+\sum_{k=1}^{n} \frac{1}{n-k+1}\right\} \leq C\left(\frac{\delta^{*}(\tau)}{\lambda \sqrt{T}}\right)^{2} \lambda^{3} T|\log \lambda| .
$$

Since $\bar{m}_{x_{k-1}}^{\prime \prime \prime} \in L_{1}(\mathbb{R}, d x),\left\|p_{t} \bar{m}_{x_{k-1}}^{\prime \prime \prime}\right\|_{\infty} \leq C t^{-1 / 2}$ and as $a_{k-1}$ is bounded, using again $(4.10)$

$$
\begin{aligned}
\left\|I_{2}\right\|_{\infty} & \leq C \lambda \delta^{*}(\tau)^{3} \sum_{k=1}^{n} \int_{T_{n}}^{t} d s\left(s-T_{k}\right)^{-1 / 2}+C \lambda \delta^{*}(\tau)^{4} \sum_{k=1}^{n}\left(t-T_{n}\right) \\
& \leq C\left(\frac{\delta^{*}(\tau)}{\lambda \sqrt{T}}\right)^{3} \lambda^{3} T^{3 / 2}+C\left(\frac{\delta^{*}(\tau)}{\lambda \sqrt{T}}\right)^{4} \lambda^{3} T^{2}
\end{aligned}
$$

Recalling the definition (4.2) of $V_{*}(\tau)$ and the definition (4.36) of $V_{*}^{\perp}(\tau)$, we get

$$
\left\|I_{3}\right\|_{\infty} \leq C \lambda V_{*}(\tau) V_{*}^{\perp}(\tau) \sqrt{T n_{\lambda}(\tau)} \leq C\left(\frac{V_{*}(\tau) V_{*}^{\perp}(\tau)}{\lambda^{2} \sqrt{T}}\right) \lambda^{2} \sqrt{T} .
$$

Finally, recalling (2.5),

$$
\left\|I_{4}\right\|_{\infty} \leq C \lambda \sqrt{T n_{\lambda}(\tau)} V_{*}(\tau)^{3} \leq C\left(\frac{V_{*}(\tau)}{\lambda \sqrt{T}}\right)^{3} \lambda^{3} T^{3 / 2} .
$$

Since we are restricting our considerations to the set where $\delta^{*}(\tau) \leq \lambda^{1-\zeta} \sqrt{T}$, $V_{*}(\tau) \leq \lambda^{1-\zeta} \sqrt{T}$ and $V_{*}^{\perp}(\tau) \leq \lambda^{1-\zeta}$, we get

$$
\sup _{t \in\left[T_{n}, T_{n+1}\right]}\left\|\Gamma_{7}^{(n)}(t)\right\|_{\infty} \leq C \lambda^{2-2 \zeta} \sqrt{T} \quad \text { hence } \quad\left|\eta_{n}(7)\right| \leq C \lambda^{2-2 \zeta} \sqrt{T} .
$$

Step 3. The term $\eta_{n}(8)$. In order to show $\eta_{n}(8)$ is negligible (as specified at the beginning of the present proof) we cannot use directly the a priori bounds, but we use equation (5.6) to get

$$
\eta_{n}(8)=\sum_{\substack{i=1 \\ i \neq 3,8}}^{10} \eta_{n}(8, i)+\eta_{n}(8,3+8)
$$

and show each of the terms on the r.h.s. above are negligible.

We have

$$
\eta_{n}(8)=\sum_{k=0}^{n-1} A_{n, k}^{(1)} \psi_{k}
$$

where, recalling $2=\left\langle 1, \bar{m}_{x_{k}}^{\prime}\right\rangle$,

$$
A_{n, k}^{(1)}:=-\frac{3 \lambda}{4} \int_{T_{n}}^{T_{n+1}} d s
$$




$$
\left\{\left\langle\bar{m}_{x_{n}}^{\prime}, p_{s-T_{k+1}} \bar{m}_{x_{k}}^{\prime}\right\rangle-\left\langle\bar{m}_{x_{n}}^{\prime}, 1\right\rangle \frac{1}{\sqrt{2 \pi\left(s-T_{k+1}\right)}}\left\langle 1, \bar{m}_{x_{k}}^{\prime}\right\rangle\right\} \mathbf{1}_{k<n} .
$$

Let us define (recall that $\bar{m}^{\prime}>0$ )

$$
B_{n, k}^{(1)}:=\lambda \int_{T_{n}}^{T_{n+1}} d s \int d x \int d y \bar{m}_{x_{n}}^{\prime}(x)\left|p_{s-T_{k+1}}(x-y)-\frac{1}{\sqrt{2 \pi\left(s-T_{k+1}\right)}}\right| \bar{m}_{x_{k}}^{\prime}(y) .
$$

We are going to prove that for any $\tau>0$ there is a constant $C=C(\tau)$ such that for any $0 \leq k<n \leq n_{\lambda}(\tau)$

$$
\left|A_{n, k}^{(1)}\right| \leq C B_{n, k}^{(1)} \leq C \lambda\left[1+x_{n+1, *}^{2}\right]\left\{\frac{1}{\sqrt{T}(n-k)^{3 / 2}}+\mathbf{1}_{k=n-1}\right\} .
$$

Proof of (6.20). Let $x_{n, k}:=x_{n}-x_{k}$ and define, for any $\theta \in \mathbb{R}$ and $t>0$,

$$
f(t, \theta)=\int d x \int d y \bar{m}^{\prime}(x) \bar{m}^{\prime}(y)\left|\frac{e^{-(y-x-\theta)^{2} / 2 t}-1}{\sqrt{2 \pi t}}\right| .
$$

Then, after a change of variables in (6.19), its r.h.s. becomes equal to $\lambda \int_{T_{n}}^{T_{n+1}} d s$ $f\left(s-T_{k+1}, x_{n, k}\right)$.

The case $k<n-1$. After changing variables in the time integral, we get, for $k<n-1$,

$$
B_{n, k}^{(1)}=\lambda T \int_{0}^{1} d t f\left(T(t+n-1-k), x_{n, k}\right) .
$$

Since $\left|e^{-|\xi|}-1\right| \leq|\xi|$ and $\bar{m}^{\prime}(x)$ decays exponentially to 0 as $|x| \rightarrow \infty$, we get

$$
f(t, \theta) \leq C \frac{1+\theta^{2}}{t^{3 / 2}}
$$

and, from (6.21),

$$
B_{n, k}^{(1)} \leq C \lambda T^{-1 / 2}\left[1+x_{n, k}^{2}\right] \int_{0}^{1} d t \frac{1}{(t+n-1-k)^{3 / 2}}
$$

which proves (6.20) when $k<n-1$.

The case $k=n-1$. We have, after a change of variables in the time integral,

$$
B_{n, n-1}^{(1)}=\lambda \int_{0}^{T} d t f\left(t, x_{n, n-1}\right) .
$$

Using the inequality $f(t, \theta) \leq 4(2 \pi t)^{-1 / 2}$ when $t \leq 1$ and (6.22) when $t>1$, we get

$$
B_{n, n-1}^{(1)} \leq \lambda \int_{0}^{1} d t \frac{4}{\sqrt{2 \pi t}}+C \lambda \int_{1}^{T} d t \frac{1+x_{n, n-1}^{2}}{t^{3 / 2}} \leq C \lambda\left(1+x_{n, *}^{2}\right) .
$$


The proof of (6.20) is concluded.

Recall now that

$$
\eta_{n}(8, i)=-\frac{3}{4} \sum_{k=0}^{n-1} A_{n, k}^{(1)}\left\langle\bar{m}_{x_{k}}^{\prime}, \Gamma_{i}^{k}\left(T_{k+1}\right)\right\rangle, \quad i=1,2,3+8,4,5,6,7,9,10 .
$$

The term $\eta_{n}(8,1)$ in $(6.16)$ vanishes by the same argument as in (6.8). Since we are working in the set $\mathcal{B}_{\lambda, \tau}^{(1)}$, see (4.37), we have

$$
\begin{array}{r}
\left|\eta_{n}(8,2)\right| \leq C \lambda\left(1+x_{n+1, *}^{2}\right)\left\{\sum_{k=0}^{n-2} \frac{1}{\sqrt{T}(n-k)^{3 / 2}}+1\right\} \lambda^{1-\zeta} \sqrt{T} \\
\leq C \lambda^{2-\zeta}\left(1+x_{n+1, *}^{2}\right)
\end{array}
$$

which is negligible for $\zeta$ small.

To bound $\eta_{n}(8,3+8)$ use (6.17), (5.2), and (5.7) to write

$$
\eta_{n}(8,3+8)=\lambda \sum_{k=0}^{n-1} A_{n, k}^{(1)} \sum_{h=0}^{k-1} B_{k, h} \psi_{h}, \quad B_{k, h}:=\int_{T_{k}}^{T_{k+1}} d s\left\langle\bar{m}_{x_{k}}^{\prime}, p_{s-T_{h+1}} \bar{m}_{x_{h}}^{\prime}\right\rangle .
$$

We note that, by (5.7), $|\psi|_{n} \leq C V_{n, *}$; it is also easy to verify $B_{k, h} \leq C \sqrt{T}(k-$ $h)^{-1 / 2}$. Plugging this bounds, together with (6.20), into (6.24), we get

$$
\begin{array}{r}
\left|\eta_{n}(8,3+8)\right| \leq C \lambda^{2} \sqrt{T} V_{n, *}\left[1+x_{n+1, *}^{2}\right] \sum_{k=0}^{n-1}\left\{\frac{1}{\sqrt{T}(n-k)^{3 / 2}}+\mathbf{1}_{k=n-1}\right\} \\
\times \sum_{h=0}^{k-1} \frac{1}{\sqrt{k-h}} \leq C \lambda V_{n, *}\left[1+x_{n+1, *}^{2}\right] \sum_{k=0}^{n-1}\left\{\frac{1}{\sqrt{T}(n-k)^{3 / 2}}+\mathbf{1}_{k=n-1}\right\} \\
\leq C \lambda V_{n, *}\left[1+x_{n+1, *}^{2}\right]
\end{array}
$$

which shows $\eta_{n}(8,3+8)$ is negligible.

It remains to bound $\eta_{n}(8, i), i=4,5,6,7,9,10$. The negligibility of those terms follows directly from (6.17), (6.20), and the bounds (4.18)-(4.22), (6.15). We thus conclude

$$
\left|\eta_{n}(8)\right| \leq C \lambda^{2-\zeta} \sqrt{T}\left[1+x_{n+1, *}^{2}\right] .
$$

Step 4. The term $\eta_{n}(4+5)$. We claim

$$
\eta_{n}(4+5)=\frac{3 \lambda}{4} \int_{T_{n}}^{T_{n+1}} d s\left\langle\bar{m}_{x_{n}}^{\prime}, p_{T_{n+1}-s} v^{(n)}(s)\right\rangle
$$


Indeed

$$
\begin{aligned}
\eta_{n}(4) & =-\frac{3 \lambda}{4} \int_{T_{n}}^{T_{n+1}} d s \int_{T_{n}}^{s} d s^{\prime}\left\langle\bar{m}_{x_{n}}^{\prime}, \frac{\partial p_{s-s^{\prime}}}{\partial s^{\prime}} v^{(n)}\left(s^{\prime}\right)\right\rangle \\
& =\frac{3 \lambda}{4} \int_{T_{n}}^{T_{n+1}} d s^{\prime} \int_{s^{\prime}}^{T_{n+1}} d s\left\langle\bar{m}_{x_{n}}^{\prime}, \frac{\partial p_{s-s^{\prime}}}{\partial s} v^{(n)}\left(s^{\prime}\right)\right\rangle \\
& =\frac{3 \lambda}{4} \int_{T_{n}}^{T_{n+1}} d s^{\prime}\left\langle\bar{m}_{x_{n}}^{\prime}, p_{T_{n+1}-s^{\prime}} v^{(n)}\left(s^{\prime}\right)\right\rangle-\eta_{n}(5)
\end{aligned}
$$

which proves (6.27).

In order to bound $\eta_{n}(4+5)$, we use equation (5.6) to get

$$
\begin{aligned}
\eta_{n}(4+5)= & \eta_{n}(4+5,2)+\eta_{n}(4+5,3,2)+\eta_{n}(4+5,3,3) \\
& +\sum_{\substack{i=1 \\
i \neq 2,3}}^{10}\left[\eta_{n}(4+5, i)+\eta_{n}(4+5,3, i)\right] .
\end{aligned}
$$

The term $\eta_{n}(4+5,2)$ is in $\sigma_{n}$. Postponing the analysis of $\eta_{n}(4+5,3,2)$ let us first show that $\eta_{n}(4+5, i), i \neq 2,3$ are negligible.

The terms $\eta_{n}(4+5, i), i \neq 2,3$. We have

$$
\eta_{n}(4+5,1)=\frac{3 \lambda}{4} \int_{T_{n}}^{T_{n+1}} d s\left\langle\bar{m}_{x_{n}}^{\prime}, p_{T_{n+1}-s} g_{s-T_{n}}^{(n)} v^{(n)}\left(T_{n}\right)\right\rangle
$$

since $v^{(n)}\left(T_{n}\right)$ is orthogonal to $\bar{m}_{x_{n}}^{\prime}$, see (3.2), we can apply (4.11) to deduce that

$$
\left|\eta_{n}(4+5,1)\right| \leq C \lambda \int_{T_{n}}^{T_{n+1}} d s e^{-\alpha\left(s-T_{n}\right)} \lambda^{1-2 \zeta}
$$

having used that $\left\|v^{(n)}\left(T_{n}\right)\right\|_{\infty} \leq C \lambda^{1-2 \zeta}$, see (4.47). Thus $\left|\eta_{n}(4+5,1)\right| \leq C \lambda^{2-2 \zeta}$ which for $\zeta$ small is negligible.

We next show $\eta_{n}(4+5,8)$ is negligible. We have

$$
\begin{aligned}
& \eta_{n}(4+5,8)=\frac{3}{4} \lambda^{2} \sum_{k=0}^{n-1} \psi_{k} \int_{T_{n}}^{T_{n+1}} d t \int_{T_{n}}^{t} d s \\
& \quad \times\left\langle\bar{m}_{x_{n}}^{\prime}, p_{T_{n+1}-t} g_{t-s}^{(n)}\left[p_{s-T_{k+1}} \bar{m}_{x_{k}}^{\prime}-\frac{2}{\sqrt{2 \pi\left(s-T_{k+1}\right)}}\right]\right\rangle .
\end{aligned}
$$

We now use the decomposition (4.31) for $g_{t-s}^{(n)}$ above. Recalling (6.19), the term obtained by replacing $g_{t-s}^{(n)}(x, y)$ by $(3 / 4) \bar{m}_{x_{n}}^{\prime}(x) \bar{m}_{x_{n}}^{\prime}(y)$ in $(6.29)$ is bounded 
by

$$
\begin{aligned}
& C \lambda \sum_{k=0}^{n-1} B_{n, k}^{(1)}\left|\psi_{k}\right| \int_{T_{n}}^{T_{n+1}} d t\left\langle\bar{m}_{x_{n}}^{\prime}, p_{T_{n+1}-t} \bar{m}_{x_{n}}^{\prime}\right\rangle \leq C \sqrt{T} \lambda^{2} V_{n, *}\left[1+x_{n+1, *}^{2}\right] \\
& \times \sum_{k=0}^{n-1}\left\{\frac{1}{\sqrt{T}(n-k)^{3 / 2}}+\mathbf{1}_{k=n-1}\right\} \leq C \sqrt{T} \lambda^{2} V_{n, *}\left[1+x_{n+1, *}^{2}\right]
\end{aligned}
$$

where we used (6.20). We next consider the case when we replace $g_{t-s}^{(n)}$ in (6.29) by $g_{t-s}^{(n, \perp)}$. Since, by (4.14) and (4.32),

$$
\begin{array}{r}
\left\|p_{T_{n+1}-t} g_{t-s}^{(n, \perp)} p_{s-T_{k+1}} \bar{m}_{x_{k}}^{\prime}\right\|_{\infty} \leq C e^{-\alpha(t-s)}\left\|p_{s-t_{k+1}} \bar{m}_{x_{k}}^{\prime}\right\|_{\infty} \\
\leq C e^{-\alpha(t-s)} \frac{1}{\sqrt{s-T_{k+1}}}
\end{array}
$$

we can bound (6.29) (with $g_{t-s}^{(n)}$ replaced by $g_{t-s}^{(n, \perp)}$ ) as

$$
\begin{aligned}
& C \lambda^{2} \sum_{k=0}^{n-1}\left|\psi_{k}\right| \int_{T_{n}}^{T_{n+1}} d t \int_{T_{n}}^{t} d s e^{-\alpha(t-s)} \frac{1}{\sqrt{s-T_{k+1}}} \\
& \leq C \sqrt{T} \lambda^{2} V_{n, *} \sum_{k=0}^{n-1}\left\{\mathbf{1}_{k=n-1}+\frac{1}{\sqrt{n-k}}\right\} \leq C\left[\lambda^{3-\zeta} T+\lambda^{2-\zeta} \sqrt{T}\right]
\end{aligned}
$$

which is negligible for $\zeta$ small enough.

It remains to show $\eta_{n}(4+5, i), i=4,5,6,7,9,10$ is negligible. This follows from the bounds (4.18)-(4.22), (6.15), and (6.27).

The terms $\eta_{n}(4+5,3, i)$. We write

$$
\eta_{n}(4+5,3)=\sum_{k=0}^{n-1} A_{n, k}^{(2)} \psi_{k}
$$

where

$$
A_{n, k}^{(2)}=\frac{3 \lambda^{2}}{4} \int_{T_{n}}^{T_{n+1}} d s^{\prime \prime} \int_{s^{\prime \prime}}^{T_{n+1}} d s^{\prime}\left\langle\bar{m}_{x_{n}}^{\prime}, p_{T_{n+1}-s^{\prime}} g_{s^{\prime}-s^{\prime \prime}}^{(n)} \frac{2}{\sqrt{2 \pi\left(s^{\prime \prime}-T_{k+1}\right)}}\right\rangle \mathbf{1}_{k<n} .
$$

By using the bounds (4.14) and (4.10), it is easy to show

$$
\left|A_{n, k}^{(2)}\right| \leq C \frac{\lambda^{2} T^{3 / 2}}{\sqrt{n-k}} \mathbf{1}_{k<n} .
$$


From (6.30) we thus get

$$
\eta_{n}(4+5,3, i)=\sum_{k=0}^{n-1} A_{n, k}^{(2)} \eta_{k}(i)
$$

The first one, $\eta_{n}(4+5,3,1)$, vanishes, by (6.8). By using the bounds (4.18)-(4.22), $(6.15),(6.26)$, and $(6.32)$ it is immediate to verify $\eta_{n}(4+5,3, i)$ is negligible for $i \neq 2,3$ if $\zeta$ is chosen small enough.

To bound $\eta(4+5,3,3)$ we note that, by $(5.12)$

$$
\eta(4+5,3,3)=-A^{(2)} A \psi
$$

with $A=\left(\left(A_{n, k}\right)\right)$ as in (5.11). Hence, recalling (5.16),

$$
\eta(4+5,3,3)=-A^{(2)}(1+A)^{-1} A \eta .
$$

By (6.32), it is easy to show

$$
\sup _{n \leq n_{\lambda}(\tau)}\left\|A^{(2)}\right\|_{n} \leq C \lambda T
$$

so that, by the bound in (5.16),

$$
\left|\eta_{n}(4+5,3,3)\right| \leq C \lambda T \sum_{i \neq 3} \max _{\ell \leq n}\left|\sum_{k<\ell} A_{\ell, k} \eta_{k}(i)\right| .
$$

By definitions (6.2) and (6.6) the term with $i=2$ in the r.h.s. of (6.47) is therefore negligible. For $i=1,7,8,10$ (resp. $i=4,5,6,9$ ) we can use the bounds already proved (6.8), (6.15), (6.26), and (6.9) (resp. the a priori bounds (4.18)-(4.21)) together with $\|A\|_{n} \leq C$ to conclude that $\eta_{n}(4+5,3,3)$ is also negligible.

We are thus left with the term $\eta_{n}(4+5,3,2)$. Recalling $(6.33)$, we write it as

$$
\eta_{n}(4+5,3,2)=\sum_{k=0}^{n-1} A_{n, k}^{(2, L)} \eta_{k}(2)+\sum_{k=0}^{n-1} A_{n, k}^{(2, R)} \eta_{k}(2)
$$

where

$$
\begin{aligned}
& A_{n, k}^{(2, L)}:= \frac{3 \lambda^{2}}{4} \frac{2}{\sqrt{2 \pi\left(T_{n+1}-T_{k+1}\right)}} \int_{T_{n}}^{T_{n+1}} d s^{\prime \prime} \int_{s^{\prime \prime}}^{T_{n+1}} d s^{\prime}\left\langle\bar{m}_{x_{n}}^{\prime}, p_{T_{n+1}-s^{\prime}} g_{s^{\prime}-s^{\prime \prime}}^{(n)} 1\right\rangle \mathbf{1}_{k<n} \\
& A_{n, k}^{(2, R)}:=\frac{3 \lambda^{2}}{4} \int_{T_{n}}^{T_{n+1}} d s^{\prime \prime} \int_{s^{\prime \prime}}^{T_{n+1}} d s^{\prime}\left[\frac{2}{\sqrt{2 \pi\left(s^{\prime \prime}-T_{k+1}\right)}}-\frac{2}{\sqrt{2 \pi\left(T_{n+1}-T_{k+1}\right)}}\right] \\
& \times\left\langle\bar{m}_{x_{n}}^{\prime}, p_{T_{n+1}-s^{\prime}} g_{s^{\prime}-s^{\prime \prime}}^{(n)} 1\right\rangle \mathbf{1}_{k<n} .
\end{aligned}
$$


Since, by (4.10)

$$
\left|\int_{T_{n}}^{T_{n+1}} d s^{\prime \prime} \int_{s^{\prime \prime}}^{T_{n+1}} d s^{\prime}\left\langle\bar{m}_{x_{n}}^{\prime}, p_{T_{n+1}-s^{\prime}} g_{s^{\prime}-s^{\prime \prime}}^{(n)} 1\right\rangle\right| \leq C T^{2}
$$

we have

$$
\left|\sum_{k=0}^{n-1} A_{n, k}^{(2, L)} \eta_{k}(2)\right| \leq C \lambda T\left|\sum_{k=0}^{n-1} \frac{\lambda \sqrt{T}}{\sqrt{n-k}} \eta_{k}(2)\right|
$$

which is negligible because we are in the set $\mathcal{B}_{\lambda, \tau}^{(4)}$, recall (6.3). On the other hand, by using again (4.10) we get

$$
\left|A_{n, k}^{(2, R)}\right| \leq C \lambda^{2} T^{3 / 2}\left[\mathbf{1}_{k=n-1}+\frac{1}{(n-k-1)^{3 / 2}}\right]
$$

whence, recalling (4.5)

$$
\left|\sum_{k=0}^{n-1} A_{n, k}^{(2, R)} \eta_{k}(2)\right| \leq C \lambda^{3-\zeta} T^{2}\left\{1+\sum_{k=0}^{n-2} \frac{1}{(n-k-1)^{3 / 2}}\right\}
$$

which is negligible.

Step 5. The term $\eta_{n}(6)$. To study this term we are going to use the same strategy as for $\eta_{n}(4+5)$. The explicit expression of $\eta_{n}(6)$ is

$$
\eta_{n}(6)=\frac{3 \lambda}{4} \sum_{k=0}^{n-1} \int_{T_{n}}^{T_{n+1}} d s \int_{T_{k}}^{T_{k+1}} d s^{\prime}\left\langle\bar{m}_{x_{n}}^{\prime}, \frac{\partial p_{s-s^{\prime}}}{\partial s} v^{(k)}\left(s^{\prime}\right)\right\rangle
$$

which we decompose as we did for $\eta_{n}(4+5)$, i.e. as in $(6.28)$ with $4+5$ replaced by 6 .

The terms $\eta_{n}(6, i), i \neq 2,3$. We have

$$
\eta_{n}(6,1)=\frac{3 \lambda}{4} \sum_{k=0}^{n-1} \int_{T_{n}}^{T_{n+1}} d s \int_{T_{k}}^{T_{k+1}} d s^{\prime}\left\langle\bar{m}_{x_{n}}^{\prime}, \frac{\partial p_{s-s^{\prime}}}{\partial s} g_{s^{\prime}-T_{k}}^{(k)} v^{(k)}\left(T_{k}\right)\right\rangle
$$

Since, by (3.2) and (4.7) $\left\|g_{s^{\prime}-T_{k}}^{(k)} v^{(k)}\left(T_{k}\right)\right\|_{\infty} \leq C e^{-\alpha\left(s^{\prime}-T_{k}\right)} \lambda^{1-\zeta} \sqrt{T}$, by (4.17) the double integral above for $k=n-1$ is less or equal than

$$
\begin{array}{rl}
\int_{T_{n}}^{T_{n+1}} & d s \int_{T_{n-1}}^{T_{n-1}+T / 2} d s^{\prime} \frac{C e^{-\alpha\left(s^{\prime}-T_{n-1}\right)} \lambda^{1-\zeta} \sqrt{T}}{s-\left[T_{n-1}+T / 2\right]} \\
& +\int_{T_{n}}^{T_{n+1}} d s \int_{T_{n-1}+T / 2}^{T_{n}} d s^{\prime} \frac{C e^{-\alpha T / 2} \lambda^{1-\zeta} \sqrt{T}}{s-s^{\prime}} \leq C \lambda^{1-\zeta \sqrt{T}}
\end{array}
$$


On the other hand, for $k<n-1$ the double integral in (6.43) is bounded by $C(n-k+1)^{-1} \lambda^{1-\zeta} \sqrt{T}$, so that

$$
\left|\eta_{n}(6,1)\right| \leq C \lambda^{2-\zeta} \sqrt{T}|\log \lambda|
$$

which is therefore negligible.

We next consider $\eta_{n}(6,8)$; its explicit expression is

$$
\begin{aligned}
& \eta_{n}(6,8)=\frac{3 \lambda^{2}}{4} \sum_{k=0}^{n-1} \sum_{h=0}^{k-1} \psi_{h} \int_{T_{n}}^{T_{n+1}} d t \int_{T_{k}}^{T_{k+1}} d s \int_{T_{k}}^{s} d s^{\prime} \\
& \times\left\langle\bar{m}_{x_{n}}^{\prime}, \frac{\partial p_{t-s}}{\partial t} g_{s-s^{\prime}}^{(k)}\left[p_{s^{\prime}-T_{h+1}} \bar{m}_{x_{h}}^{\prime}-\frac{2}{\sqrt{2 \pi\left(s^{\prime}-T_{h+1}\right)}}\right]\right\rangle .
\end{aligned}
$$

We now use the decomposition (4.31) for $g_{t-s}^{(n)}$ above. Since

$$
\left|\left\langle\bar{m}_{x_{n}}^{\prime}, \frac{\partial p_{t-s}}{\partial t} \bar{m}_{x_{k}}^{\prime}\right\rangle\right| \leq \frac{C}{(t-s)^{3 / 2}}
$$

and recalling (6.19), the term obtained replacing $g_{s-s^{\prime}}^{(k)}(x, y)$ in $(6.45)$ by $(3 / 4) \bar{m}_{x_{k}}^{\prime}(x) \bar{m}_{x_{k}}^{\prime}(y)$ can be bounded by

$$
C \lambda V_{n, *} \sum_{k=0}^{n-1} \sum_{h=0}^{k-1} \frac{\sqrt{T}}{(n-k)^{3 / 2}} B_{k, h}^{(1)} \leq C \lambda^{3-\zeta} T\left[1+x_{n+1, *}\right]
$$

which is negligible. We next consider the case when $g_{s-s^{\prime}}^{(k)}$ in $(6.45)$ is replaced by $g_{s-s^{\prime}}^{(k, \perp)}$. By (4.17), (4.32), and (4.14) we have

$$
\left\|\frac{\partial p_{t-s}}{\partial t} g_{s-s^{\prime}}^{(k, \perp)}\left[p_{s^{\prime}-T_{h+1}} \bar{m}_{x_{h}}^{\prime}-\frac{2}{\sqrt{2 \pi\left(s^{\prime}-T_{h+1}\right)}}\right]\right\|_{\infty} \leq C \frac{e^{-\alpha\left(s-s^{\prime}\right)}}{t-s} \frac{1}{\sqrt{s^{\prime}-T_{h+1}}}
$$

hence the r.h.s. of $(6.45)$ with $g_{t-s}^{(k)}$ replaced by $g_{t-s}^{(k, \perp)}$ can be bounded by

$$
C \lambda^{2} \sqrt{T} V_{n, *} \sum_{k=0}^{n-1} \sum_{h=0}^{k-1} \frac{1}{(n-k) \sqrt{k-h}} \leq C \lambda^{2-\zeta}|\log \lambda| \sqrt{T}
$$

which is negligible.

It remains to consider the terms $\eta_{n}(6, i), i \geq 4, i \neq 8$. We have

$$
\left|\eta_{n}(6, i)\right| \leq C \lambda|\log \lambda| T \max _{k \leq n} \sup _{t \in\left[T_{k}, T_{k+1}\right]}\left\|\Gamma_{i}^{(k)}(t)\right\|_{\infty}
$$

which, together with (4.18) for $i=4$, (4.19) for $i=5$, (4.20) for $i=6$, (6.15) for $i=7,(4.21)$ for $i=9,(6.9)$ for $i=10$, shows that the terms $\eta_{n}(6, i), i \geq 4, i \neq 8$ are all negligible. 
The terms $\eta_{n}(6,3, i)$. The term $\eta_{n}(6,3,1)=0$. Analogously to $(6.30)$, we write

$$
\eta_{n}(6,3)=\sum_{k=0}^{n-1} A_{n, k}^{(3)} \psi_{k}
$$

where

$$
\begin{array}{rl}
A_{n, k}^{(3)}=\frac{3 \lambda^{2}}{4} \sum_{h=k+2}^{n} \int_{T_{n}}^{T_{n+1}} & d t \int_{T_{h-1}}^{T_{h}} d s \int_{T_{h-1}}^{s} d s^{\prime} \\
& \times\left\langle\bar{m}_{x_{n}}^{\prime}, \frac{\partial p_{t-s}}{\partial t} g_{s-s^{\prime}}^{(h-1)} \frac{2}{\sqrt{2 \pi\left(s^{\prime}-T_{k+1}\right)}}\right\rangle \mathbf{1}_{k<n-1}
\end{array}
$$

By (4.17), (4.10), and (4.14) it is easy to show

$$
\left|A_{n, k}^{(3)}\right| \leq C \frac{\lambda^{2} T^{3 / 2} \log (n-k)}{\sqrt{n-k}} \mathbf{1}_{k<n-1}
$$

Except for the factor $\log (n-k) \leq C|\log \lambda|, A_{n, k}^{(3)}$ has the same behaviour as $A_{n, k}^{(2)}$, compare (6.32) with (6.50). The analysis in Step 4 applies and we can therefore conclude that all the terms $\eta_{n}(6,3, i), i \neq 2$, are negligible.

We are left with $\eta_{n}(6,3,2)$. As in (6.38) we define

$$
\begin{aligned}
& \begin{aligned}
A_{n, k}^{(3, L)}:=\frac{3 \lambda^{2}}{4} \sum_{h=k+2}^{n} \frac{2}{\sqrt{2 \pi\left(T_{h+1}-T_{k+1}\right)}} \int_{T_{n}}^{T_{n+1}} d t \int_{T_{h-1}}^{T_{h}} d s \int_{T_{h-1}}^{s} d s^{\prime} \\
\quad \times\left\langle\bar{m}_{x_{n}}^{\prime}, \frac{\partial p_{t-s}}{\partial t} g_{s-s^{\prime}}^{(h-1)} 1\right\rangle \mathbf{1}_{k<n-1}
\end{aligned} \\
& A_{n, k}^{(3, R)}:=\frac{3 \lambda^{2}}{4} \sum_{h=k+2}^{n} \int_{T_{n}}^{T_{n+1}} d t \int_{T_{h-1}}^{T_{h}} d s \int_{T_{h-1}}^{s} d s^{\prime} \\
& {\left[\frac{2}{\sqrt{2 \pi\left(s^{\prime}-T_{k+1}\right)}}-\frac{2}{\sqrt{2 \pi\left(T_{h+1}-T_{k+1}\right)}}\right]\left\langle\bar{m}_{x_{n}}^{\prime}, \frac{\partial p_{t-s}}{\partial t} g_{s-s^{\prime}}^{(h-1)} 1\right\rangle \mathbf{1}_{k<n-1}}
\end{aligned}
$$

Setting

$$
B_{n, h}^{(2)}:=\frac{3 \lambda}{2 \sqrt{2 \pi} T} \int_{T_{n}}^{T_{n+1}} d t \int_{T_{h-1}}^{T_{h}} d s \int_{T_{h-1}}^{s} d s^{\prime}\left\langle\bar{m}_{x_{n}}^{\prime}, \frac{\partial p_{t-s}}{\partial t} g_{s-s^{\prime}}^{(h-1)} 1\right\rangle
$$

we have

$$
\left|\sum_{j=0}^{n-1} A_{n, j}^{(3, L)} \eta_{j}(2)\right| \leq \sum_{h=2}^{n}\left|B_{n, h}^{(2)}\right|\left|\sum_{j=0}^{h-2} \frac{\lambda \sqrt{T}}{\sqrt{h-j}} \eta_{j}(2)\right|
$$


and, using (4.10) and (4.17),

$$
\left|B_{n, h}^{(2)}\right| \leq C \frac{\lambda T}{n-h} .
$$

Thus, since we are in the set $\mathcal{B}_{\lambda, \tau}^{(4)}$, see (6.3), the l.h.s. of (6.53) is negligible. On the other hand, by using again (4.10) and (4.17) it is easy to show

$$
\left|A_{n, k}^{(3, R)}\right| \leq C \lambda^{2}|\log \lambda| T^{3 / 2} \frac{1}{(n-k)^{3 / 2}}
$$

hence

$$
\left|\sum_{j=0}^{n-1} A_{n, j}^{(3, R)} \eta_{j}(2)\right| \leq C \lambda^{3-\zeta}|\log \lambda| T^{2} \sum_{j=0}^{n-1} \frac{1}{(n-j)^{3 / 2}}
$$

which is negligible.

Step 6. The term $\eta_{n}(9)$. The iterative scheme to estimate $\eta(9)$ is the following. We first branch, using (5.6), one of the $v^{(n)}(s)$ which appears on the r.h.s. of $(3.14)$ and stop the iteration for the terms $\eta(9 \mid i), i \neq 2,3$. In $\eta(9 \mid 3)$ we use again the equation (5.6) and stop the iteration. We then branch the other $v^{(n)}(s)$ on the term $\eta(9 \mid 2)$ stopping the iteration with the same rule as before. We thus get

$$
\begin{aligned}
\eta(9)= & \eta(9 \mid 2 ; 2)+\eta(9 \mid 2 ; 3,2)+\eta(9 \mid 2 ; 3,3)+\eta(9 \mid 3,2)+\eta(9 \mid 3,3) \\
& +\sum_{i \neq 2,3}[\eta(9 \mid i)+\eta(9 \mid 2 ; i)+\eta(9 \mid 2 ; 3, i)+\eta(9 \mid 3, i)]
\end{aligned}
$$

We need to show that $\eta(9)-\eta(9 \mid 2 ; 2)$ is negligible. We start from $\eta(9 \mid i)$, $i \neq 2,3$. We have

$$
\eta_{n}(9 \mid i)=\frac{9}{4} \int_{T_{n}}^{T_{n+1}} d t\left\langle\bar{m}_{x_{n}}^{\prime}, \bar{m}_{x_{n}} v^{(n)}(t) \Gamma_{i}^{(n)}(t)\right\rangle .
$$

For $i=1$, by $(3.2),\left\|g_{t-T_{n}}^{(n)} v^{(n)}\left(T_{n}\right)\right\|_{\infty} \leq C e^{-\alpha\left(t-T_{n}\right)}\left\|v^{(n)}\left(T_{n}\right)\right\|_{\infty}$, by $(4.47)$ $\left\|v^{(n)}\left(T_{n}\right)\right\|_{\infty} \leq \lambda^{1-\zeta}$, hence

$$
\left|\eta_{n}(9 \mid 1)\right| \leq C \lambda^{1-\zeta} \sqrt{T} \lambda^{1-\zeta}
$$

so that it is negligible. On the set $\mathcal{B}_{\lambda, \tau}^{(1,2)}$, see (4.37) and (6.6), we can use the bounds (4.7), (4.18), (4.19), (4.20), (6.15), (4.21), and (6.9), which show $\eta(9 \mid i)$ is negligible for $i=4,5,6,7,9,10$. To bound $\eta(9 \mid 8)$ we note that, by (5.5), (4.7), (using also $\|\bar{m}\|_{\infty}=1, \bar{m}^{\prime}(x)>0$, and $g_{t}^{(n)}(x, y) \geq 0$ ), we have

$$
\left|\eta_{n}(9 \mid 8)\right| \leq \lambda C V_{n, *} \sum_{k=0}^{n-1}\left|\psi_{k}\right| \int_{T_{n}}^{T_{n+1}} d t \int_{T_{n}}^{t} d s \frac{1}{\sqrt{s-T_{k+1}}}
$$




$$
\times \int d x d y d z \bar{m}_{x_{n}}^{\prime}(x) g_{t-s}^{(n)}(x, y)\left|e^{-(y-z)^{2} /\left[2\left(s-T_{k+1}\right)\right]}-1\right| \bar{m}_{x_{k}}^{\prime}(z) .
$$

Since $g_{t}^{(n)}$ is self-adjoint in $L_{2}(\mathbb{R}, d x)$ we have $\int d x \bar{m}_{x_{n}}^{\prime}(x) g_{t-s}^{(n)}(x, y)=\bar{m}_{x_{n}}^{\prime}(y)$. Then, from (6.19) and (6.20) it follows that

$$
\left|\eta_{n}(9 \mid 8)\right| \leq C V_{*}(\tau) T \sum_{k=0}^{n-1} B_{n, k}^{(1)}\left|\psi_{k}\right| \leq C \lambda V_{*}(\tau)^{2} T\left(1+x_{n+1, *}^{2}\right)
$$

which is negligible because of (4.7).

We next consider $\eta(9 \mid 3, i), i \neq 2,3$,

$$
\eta_{n}(9 \mid 3, i)=\frac{9 \lambda}{4} \sum_{k=0}^{n-1} \eta_{k}(i) \int_{T_{n}}^{T_{n+1}} d t \int_{T_{n}}^{t} d s\left\langle\bar{m}_{x_{n}}^{\prime}, \bar{m}_{x_{n}} v^{(n)}(s) g_{t-s}^{(n)} \frac{2}{\sqrt{2 \pi\left(s-T_{k+1}\right)}}\right\rangle .
$$

Note that, from $(6.8) \eta(9 \mid 3,1)=0$ and that, for $i \geq 4$, from (5.11),

$$
\begin{array}{r}
\left|\eta_{n}(9 \mid 3, i)\right| \leq C \lambda V_{n, *} \sum_{k=0}^{n-1}\left|\eta_{k}(i)\right| \int_{T_{n}}^{T_{n+1}} d t \int_{T_{n}}^{t} d s \frac{1}{\sqrt{s-T_{k+1}}} \\
\leq T C V_{n, *} \sum_{k=0}^{n-1} A_{n, k}\left|\eta_{k}(i)\right| .
\end{array}
$$

Since $\|A\|_{n} \leq C$, by using again (4.7), (4.18), (4.19), and (4.20)-(6.9), we show $\eta(9 \mid 3, i), i \geq 4$ is negligible. Next, using (5.12), (5.16) and (6.59),

$$
\begin{aligned}
\eta(9 \mid 3,3)=-\frac{9 \lambda}{4} \sum_{k=0}^{n-1}((1 & \left.+A)^{-1} A \eta\right)_{k} \int_{T_{n}}^{T_{n+1}} d t \int_{T_{n}}^{t} d s \\
& \times\left\langle\bar{m}_{x_{n}}^{\prime}, \bar{m}_{x_{n}} v^{(n)}(s) g_{t-s}^{(n)} \frac{2}{\sqrt{2 \pi\left(s-T_{k+1}\right)}}\right\rangle
\end{aligned}
$$

whence, recalling $\left\|(1+A)^{-1}\right\|_{n} \leq C,\|A\|_{n} \leq C, \eta_{n}(1)=0$,

$$
\begin{aligned}
|\eta(9 \mid 3,3)|_{n} & \leq C \lambda T\left(\sup _{k \leq n}\left|(A \eta(2))_{k}\right|+\sum_{i=4}^{10}|\eta(i)|_{n}\right) V_{n, *} \sum_{k=0}^{n-1} \frac{\sqrt{T}}{\sqrt{n-k}} \\
& \leq C T\left[\lambda^{2-\zeta} T+\lambda^{2-2 \zeta} T^{2}\left(1+x_{n+1, *}^{2}\right)\right] V_{n, *}
\end{aligned}
$$

where we used the bounds (6.8), (6.15), (6.26), (6.9), and (4.18)-(4.21) to estimate $|\eta(i)|$ together with the fact that we are on the set $\mathcal{B}_{\lambda, \tau}$ (see (6.2) and (6.6)). Hence $\eta(9 \mid 3,3)$ is negligible.

Since on the set $\mathcal{B}_{\lambda, \tau} \Gamma_{2}^{(n)}(t)$ has the same order as $v^{(n)}(t)$ (see (4.5)) we can bound $\eta(9 \mid 2 ; i), \eta(9 \mid 2 ; 3, i), i \neq 2,3$, and $\eta(9 \mid 2 ; 3,3)$ as above. 
We are left with the bounds of $\eta(9 \mid 3,2)$ and $\eta(9 \mid 2 ; 3,2)$. We have $\eta_{n}(9 \mid 3,2)=$ $\eta_{n}^{(L)}(9 \mid 3,2)+\eta_{n}^{(R)}(9 \mid 3,2)$ where

$$
\begin{aligned}
& \eta_{n}^{(L)}(9 \mid 3,2):= \frac{9 \lambda}{4} \sum_{k=0}^{n-1} \frac{2}{\sqrt{2 \pi\left(T_{n+1}-T_{k+1}\right)}} \eta_{k}(2) \\
& \int_{T_{n}}^{T_{n+1}} d t \int_{T_{n}}^{t} d s\left\langle\bar{m}_{x_{n}}^{\prime}, \bar{m}_{x_{n}} v^{(n)}(s) g_{t-s}^{(n)} 1\right\rangle \\
& \eta_{n}^{(R)}(9 \mid 3,2):=\frac{9 \lambda}{4} \sum_{k=0}^{n-1} \eta_{k}(2) \\
& \times \int_{T_{n}}^{T_{n+1}} d t \int_{T_{n}}^{t} d s\left[\frac{2}{\sqrt{2 \pi\left(s-T_{k+1}\right)}}-\frac{2}{\sqrt{2 \pi\left(T_{n+1}-T_{k+1}\right)}}\right] \\
& \quad \times\left\langle\bar{m}_{x_{n}}^{\prime}, \bar{m}_{x_{n}} v^{(n)}(s) g_{t-s}^{(n)} 1\right\rangle .
\end{aligned}
$$

Since, by (4.7) and (4.10),

$$
\left|\int_{T_{n}}^{T_{n+1}} d t \int_{T_{n}}^{t} d s\left\langle\bar{m}_{x_{n}}^{\prime}, \bar{m}_{x_{n}} v^{(n)}(s) g_{t-s}^{(n)} 1\right\rangle\right| \leq C \lambda^{1-\zeta} T^{5 / 2}
$$

we have

$$
\left|\eta_{n}^{(L)}(9 \mid 3,2)\right| \leq C \lambda^{1-\zeta} T^{3 / 2}\left|\sum_{k=0}^{n-1} \frac{\lambda \sqrt{T}}{\sqrt{n-k}} \eta_{k}(2)\right|
$$

which is negligible since we are in the set $\mathcal{B}_{\lambda, \tau}^{(4)}$, see (6.3). On the other hand, again by (4.7) and (4.10),

$$
\begin{gathered}
\left|\int_{T_{n}}^{T_{n+1}} d t \int_{T_{n}}^{t} d s\left[\frac{2}{\sqrt{2 \pi\left(s-T_{k+1}\right)}}-\frac{2}{\sqrt{2 \pi\left(T_{n+1}-T_{k+1}\right)}}\right]\left\langle\bar{m}_{x_{n}}^{\prime}, \bar{m}_{x_{n}} v^{(n)}(s) g_{t-s}^{(n)} 1\right\rangle\right| \\
\leq C \lambda^{1-\zeta} T^{2}\left[\mathbf{1}_{k=n-1}+\frac{1}{(n-k-1)^{3 / 2}}\right]
\end{gathered}
$$

whence, by (4.13) and (4.5)

$$
\left|\eta_{n}^{(R)}(9 \mid 3,2)\right| \leq C \lambda^{2-\zeta} T^{2}\left[\sum_{k=0}^{n-2} \frac{1}{(n-k-1)^{3 / 2}}\left|\eta_{k}(2)\right|+\left|\eta_{n-1}(2)\right|\right] \leq \lambda^{3-\zeta} T^{5 / 2}
$$

which is negligible.

Since $\Gamma_{n}^{(2)}(t)$ has the same order as $v^{(n)}(t)$, it follows from (6.56) that the term $\eta(9 \mid 2 ; 3,2)$ can be analyzed as $\eta(9 \mid 3,2)$. Proposition 6.2 is thus proved. 


\section{Bounds on the stochastic terms}

The stochastic terms in the title are the ones left from the previous section, i.e. $\sigma_{n}$, see (6.1). As already mentioned we will obtain bounds for the sums of the components of these vectors rather than for the components themselves. We define

$$
\begin{aligned}
S_{n}(\ell) & :=\sum_{k=0}^{n-1} \eta_{k}(\ell), \quad \ell \in \mathcal{L}:=\{2,(4+5,2),(6,2),(9 \mid 2 ; 2)\} \\
S_{n} & :=\sum_{\ell \in \mathcal{L}} S_{n}(\ell)=\sum_{k=0}^{n-1} \sigma_{k}
\end{aligned}
$$

and call $S$ the vector whose components are $S_{n}$. Analogously $\xi$ is the vector whose components are $\xi_{n}$, see (5.8).

Proposition 7.1 Recall that $A$ is the matrix whose entries are defined in (5.11). Then

$$
\xi=S-A S+R+A^{2} \xi \quad \text { on the set } \mathcal{B}_{\lambda, \tau}
$$

where

$$
R_{n}:=\sum_{k=0}^{n-1}\left(\eta_{k}-\sigma_{k}\right)-\sum_{k=0}^{n-1}(A[\eta-\sigma])_{k} .
$$

Moreover, for each $\tau>0$ there is a constant $C=C(\tau)$ such that for any $\lambda$ and $\zeta$ small enough

$$
|R|_{n} \leq C T^{-1 / 4}\left[1+x_{n+1, *}^{2}\right] \quad \text { on the set } \mathcal{B}_{\lambda, \tau} .
$$

The proof is based on the following simple lemma.

Lemma 7.2 Let $M$ be a lower triangular matrix such that $M_{n, k}=M_{n^{\prime}, k^{\prime}}$ whenever $n^{\prime}-k^{\prime}=n-k$. Let also $u=\left\{u_{h} ; h=0, \ldots, n\right\}$ be a vector and define $v_{n}=\sum_{h=0}^{n-1} u_{h}$ (resp. $\left.v_{0}=0\right)$. Then

$$
\sum_{h=0}^{n-1}(M u)_{h}=(M v)_{n}
$$

Proof. The l.h.s. of (7.5) can be written as

$$
\sum_{h=0}^{n-1} \sum_{k=0}^{h-1} M_{h, k}\left(v_{k+1}-v_{k}\right)=\sum_{h=0}^{n-1} \sum_{k=0}^{h-1} M_{h, k} v_{k+1}-\sum_{h=0}^{n-1} \sum_{k=0}^{h-1} M_{h, k} v_{k} .
$$

We call $k^{\prime}=k+1$ in the first sum and $h^{\prime}=h-1$ in the second one, getting

$$
\sum_{h=0}^{n-1}(M u)_{h}=\sum_{h=0}^{n-1} \sum_{k^{\prime}=1}^{h} M_{h, k^{\prime}-1} v_{k^{\prime}}-\sum_{h^{\prime}=-1}^{n-2} \sum_{k=0}^{h^{\prime}} M_{h^{\prime}+1, k} v_{k}
$$


We may as well extend the first sum from $k^{\prime}=0$ because $v_{0}=0$. We then get

$$
\sum_{h=0}^{n-1}(M u)_{h}=\sum_{k=1}^{n-1} M_{n-1, k-1} v_{k}+\sum_{h=0}^{n-2} \sum_{k=0}^{h}\left[M_{h, k-1}-M_{h+1, k}\right] v_{k} .
$$

Note that the second term above vanishes by the assumptions on $M$, and this completes the proof of (7.5).

Proof of Proposition 7.1. From (5.8) and (5.17),

$$
\xi_{n}=\sum_{k=0}^{n-1}\left[\eta-A \eta+A^{2} \psi\right]_{k}
$$

Next, apply Lemma 7.2 for $A$ and $A^{2}$. From (7.1) and (5.8), we get (7.2). Since $\|A\|_{n} \leq C$, the bound (7.4) follows from Proposition 6.2.

Our next task is to control the stochastic term $S_{n}$.

Proposition 7.3 For any $\tau>0$ we have

$$
\begin{gathered}
\lim _{L \rightarrow \infty} \varlimsup_{\lambda \downarrow 0} \mathbb{P}\left(\sup _{n \leq n_{\lambda}(\tau)}\left|S_{n}(2)\right|>L\right)=0 \\
\lim _{\lambda \downarrow 0} \mathbb{P}\left(\sup _{n \leq n_{\lambda}(\tau)} \frac{\left|S_{n}(\ell)\right|}{1+x_{n, *}}>T^{-1 / 4}\right)=0, \quad \ell \in \mathcal{L} \backslash\{2\} .
\end{gathered}
$$

Proof of (7.6). By (3.9), (5.10), and (7.1),

$$
S_{n}(2)=-\frac{3 \lambda}{4} \sum_{k=0}^{n-1}\left\langle\bar{m}_{x_{k}}^{\prime}, z^{(k)}\left(T_{k+1}\right)\right\rangle
$$

which is an $\mathcal{F}_{T_{n}}$-martingale. In Appendix $\mathrm{B}$ it is proved there is a $C$ so that, for any $n \leq n_{\lambda}(\tau)$ and $t \in\left[T_{n}+1, T_{n+1}\right]$,

$$
\sup _{x \in \mathbb{R}} \mathbb{E}\left(\left(z^{(n)}(t, x)\right)^{2 p}\right) \leq C\left(t-T_{n}\right)^{p}, \quad p=1,2 .
$$

Then, by Doob's martingale inequality, we get, for some constant $C$,

$$
\mathbb{P}\left(\sup _{n \leq n_{\lambda}(\tau)}\left|S_{n}(2)\right|>L\right) \leq \frac{1}{L^{2}} \mathbb{E}\left(S_{n_{\lambda}(\tau)}(2)^{2}\right) \leq \frac{C n_{\lambda}(\tau) \lambda^{2} T}{L^{2}}
$$

which proves (7.6). 
Proof of (7.7).

The case $\ell=(4+5,2)$. We have (recall $(6.27))$

$$
S_{n}(4+5,2)=\frac{3 \lambda^{2}}{4} \sum_{h=0}^{n-1} \int_{T_{h}}^{T_{h+1}} d s\left\langle\bar{m}_{x_{h}}^{\prime}, p_{T_{h+1}-s} z^{(h)}(s)\right\rangle .
$$

This is again a $\mathcal{F}_{T_{n}}$-martingale, so that, using again Doob's inequality, and the bound

$$
\mathbb{E}\left(\left|\int_{T_{h}}^{T_{h+1}} d s\left\langle\bar{m}_{x_{h}}^{\prime}, p_{T_{h+1}-s} z^{(h)}(s)\right\rangle\right|^{2}\right) \leq C T^{3}
$$

(which follows from (7.9)) we conclude that for each $\zeta>0$ there is a constant $C=C(\tau, \zeta)$ such that

$$
\mathbb{P}\left(\sup _{n \leq n_{\lambda}(\tau)}\left|S_{n}(4+5,2)\right|>T^{-1 / 4}\right) \leq C n_{\lambda}(\tau) \lambda^{4} T^{3} \sqrt{T} \leq C \lambda^{2} T^{5 / 2} .
$$

The case $\ell=(6,2)$. By (3.13) and (3.9)

$$
S_{n}(6,2)=\frac{3 \lambda^{2}}{4} \sum_{h=1}^{n-1} \sum_{k=1}^{h} \int_{T_{h}}^{T_{h+1}} d s \int_{T_{k-1}}^{T_{k}} d s^{\prime}\left\langle\bar{m}_{x_{h}}^{\prime}, \frac{\partial p_{s-s^{\prime}}}{\partial s} z^{(k-1)}\left(s^{\prime}\right)\right\rangle .
$$

For each $h$ and $k$ above, we write, recalling that $\bar{m}_{\xi}(x)=\bar{m}(x-\xi)$,

$$
\bar{m}_{x_{h}}^{\prime}(x)=\bar{m}_{x_{k-1}}^{\prime}(x)-\int_{x_{k-1}}^{x_{h}} d \xi \bar{m}_{\xi}^{\prime \prime}(x)
$$

thus obtaining, by exchanging the sums in (7.12),

$$
S_{n}(6,2)=-\tilde{S}_{n}(6,2)+\sum_{k=1}^{n-1} X_{n, k}
$$

where

$$
\begin{aligned}
\tilde{S}_{n}(6,2) & :=\frac{3 \lambda^{2}}{4} \sum_{h=1}^{n-1} \sum_{k=1}^{h} \int_{T_{h}}^{T_{h+1}} d s \int_{T_{k-1}}^{T_{k}} d s^{\prime} \int_{x_{k-1}}^{x_{h}} d \xi\left\langle\bar{m}_{\xi}^{\prime \prime}, \frac{\partial p_{s-s^{\prime}}}{\partial s} z^{(k-1)}\left(s^{\prime}\right)\right\rangle \\
X_{n, k} & :=\frac{3 \lambda^{2}}{4} \int_{T_{k}}^{T_{n}} d s \int_{T_{k-1}}^{T_{k}} d s^{\prime}\left\langle\bar{m}_{x_{k-1}}^{\prime}, \frac{\partial p_{s-s^{\prime}}}{\partial s} z^{(k-1)}\left(s^{\prime}\right)\right\rangle .
\end{aligned}
$$

We define

$$
M_{k}^{(n)}:=\sum_{h=1}^{k-1} X_{n, h}, \quad k=1, \ldots, n
$$


with $M_{1}^{(n)}=0$. For each fixed $n,\left\{M_{k}^{(n)} ; k=1, \ldots, n\right\}$ is an $\mathcal{F}_{T_{k-1}}$-martingale since $\mathbb{E}\left(X_{n, h} \mid \mathcal{F}_{T_{h-1}}\right)=0$. The quadratic variation of $M_{k}^{(n)}$ is

$$
\left[M^{(n)}\right]_{k}=\sum_{h=2}^{k}\left(M_{h}^{(n)}-M_{h-1}^{(n)}\right)^{2}=\sum_{h=1}^{k-1} X_{n, h}^{2} .
$$

We have

$$
\begin{gathered}
\mathbb{E}\left(X_{n, k}^{2} \mid \mathcal{F}_{T_{k-1}}\right)=\frac{9 \lambda^{4}}{16} \int_{T_{k}}^{T_{n}} d s \int_{T_{k-1}}^{T_{k}} d s^{\prime} \int_{T_{k}}^{T_{n}} d u \int_{T_{k-1}}^{T_{k}} d u^{\prime} \int_{T_{k-1}}^{s^{\prime} \wedge u^{\prime}} d s^{\prime \prime} \int d x d x^{\prime} d y \\
\times\left(\frac{\partial p_{s-s^{\prime}}}{\partial s} \bar{m}^{\prime}\right)(x)\left(\frac{\partial p_{u-u^{\prime}}}{\partial u} \bar{m}^{\prime}\right)\left(x^{\prime}\right) g_{s^{\prime}-s^{\prime \prime}}(x, y) g_{u^{\prime}-s^{\prime \prime}}\left(x^{\prime}, y\right) a\left(\lambda^{\beta}\left(y+x_{k-1}\right)\right)^{2} \\
\leq C\|a\|_{\infty}^{2} \lambda^{4} \int_{T_{k}}^{T_{n}} d s \int_{T_{k-1}}^{T_{k}} d s^{\prime} \int_{T_{k}}^{T_{n}} d u \int_{T_{k-1}}^{T_{k}} d u^{\prime} \int_{T_{k-1}}^{s^{\prime} \wedge u^{\prime}} d s^{\prime \prime} \\
\times\left\|g_{s^{\prime}-s^{\prime \prime}} \frac{\partial p_{s-s^{\prime}}}{\partial s} \bar{m}^{\prime}\right\|_{\infty}\left\|\frac{\partial p_{u-u^{\prime}}}{\partial u} \bar{m}^{\prime}\right\|_{1}\left\|g_{u^{\prime}-s^{\prime \prime}} 1\right\|_{\infty} \\
\leq C\|a\|_{\infty}^{2} \lambda^{4} \int_{T_{k}}^{T_{n}} d s \int_{T_{k-1}}^{T_{k}} d s^{\prime} \int_{T_{k}}^{T_{n}} d u \int_{T_{k-1}}^{T_{k}} d u^{\prime} \int_{T_{k-1}}^{s^{\prime} \wedge u^{\prime}} d s^{\prime \prime} \frac{1}{\left(s-s^{\prime}\right)^{3 / 2}} \frac{1}{u-u^{\prime}} \\
\leq C\|a\|_{\infty}^{2} \lambda^{4}|\log \lambda| T^{5 / 2} .
\end{gathered}
$$

Since, conditionally on $\mathcal{F}_{T_{k-1}}, z^{k-1}$ is Gaussian we also have

$$
\mathbb{E}\left(X_{n, k}^{4} \mid \mathcal{F}_{T_{k-1}}\right) \leq C\|a\|_{\infty}^{4} \lambda^{8}|\log \lambda|^{2} T^{5} .
$$

By the BDG inequality (see [16, $\S 6$, E. 4.1]) and the above bounds it follows that

$$
\begin{aligned}
\mathbb{E}\left(\left(M_{n}^{(n)}\right)^{4}\right) & \leq C \mathbb{E}\left(\left[M^{(n)}\right]_{n}^{2}\right)=C \mathbb{E}\left(\sum_{h=1}^{n-1} X_{n, h}^{2}\right)^{2} \\
& \leq C\left\{\sum_{h=1}^{n-1} \mathbb{E} X_{n, h}^{4}+2 \sum_{k=1}^{n-1} \sum_{h=1}^{k-1} \mathbb{E}\left(X_{n, h}^{2} \mathbb{E}\left(X_{n, k}^{2} \mid \mathcal{F}_{T_{k-1}}\right)\right)\right\} \\
& \leq C n^{2} \lambda^{8}|\log \lambda|^{2} T^{5} .
\end{aligned}
$$

We next use the following corollary of the Chebyshev inequality,

$$
\mathbb{P}\left(\sup _{n \leq N}\left|\vartheta_{n}\right|>\delta\right) \leq \mathbb{P}\left(\sum_{n \leq N}\left|\vartheta_{n}\right|^{p}>\delta^{p}\right) \leq \delta^{-p} \sum_{n \leq N} \mathbb{E}\left(\left|\vartheta_{n}\right|^{p}\right)
$$

and get, by (7.16), choosing $p=4$,

$$
\mathbb{P}\left(\sup _{n \leq n_{\lambda}(\tau)}\left|\sum_{k=1}^{n-1} X_{n, k}\right|>T^{-1 / 4}\right) \leq T \sum_{n=1}^{n_{\lambda}(\tau)} C n^{2} \lambda^{8}|\log \lambda|^{2} T^{5} \leq C \lambda^{2} T^{4} .
$$


It remains to bound $\tilde{S}_{n}(6,2)$. We split it into two terms by using the decomposition (4.33) for $z^{(k-1)}$. We get

$$
\begin{aligned}
& \tilde{S}_{n}^{\perp}(6,2):= \frac{3 \lambda^{2}}{4} \sum_{h=1}^{n-1} \sum_{k=1}^{h} \int_{T_{h}}^{T_{h+1}} d s \int_{T_{k-1}}^{T_{k}} d s^{\prime} \int_{x_{k-1}}^{x_{h}} d \xi\left\langle\bar{m}_{\xi}^{\prime \prime}, \frac{\partial p_{s-s^{\prime}}}{\partial s} z^{(k-1, \perp)}\left(s^{\prime}\right)\right\rangle \\
& \tilde{S}_{n}^{\|}(6,2):=\frac{3 \lambda^{2}}{4} \sum_{h=1}^{n-1} \sum_{k=1}^{h} \int_{T_{h}}^{T_{h+1}} d s \int_{T_{k-1}}^{T_{k}} d s^{\prime} \\
& \times \int_{x_{k-1}}^{x_{h}} d \xi\left\langle\bar{m}_{\xi}^{\prime \prime}, \frac{\partial p_{s-s^{\prime}}}{\partial s} \bar{m}_{x_{k-1}}^{\prime}\right\rangle w^{(k-1)}\left(s^{\prime}\right)
\end{aligned}
$$

where we set $w^{(k)}(t):=\frac{3}{4}\left\langle\bar{m}_{x_{k}}^{\prime}, z^{(k)}(t)\right\rangle$. that

In Appendix A we prove for each $\gamma \in(0,1 / 2)$ there exists a constant $C$ such

$$
\left|\left\langle\bar{m}^{\prime \prime}, \frac{\partial p_{t}}{\partial t} \varphi\right\rangle\right| \leq \frac{C}{t^{3 / 2}}\left(\|\varphi\|_{\infty} \wedge \frac{\|\varphi\|_{1}}{t^{\gamma}}\right) .
$$

We thus get

$$
\begin{aligned}
\sup _{n \leq n_{\lambda}(\tau)} \frac{\left|\tilde{S}_{n}^{\perp}(6,2)\right|}{1+x_{n, *}} & \leq C \lambda^{2} \sum_{h=1}^{n_{\lambda}(\tau)-1} \sum_{k=1}^{h} \int_{T_{h}}^{T_{h+1}} d s \int_{T_{k-1}}^{T_{k}} d s^{\prime} \frac{1}{\left(s-s^{\prime}\right)^{3 / 2}}\left\|z^{(k-1, \perp)}\left(s^{\prime}\right)\right\|_{\infty} \\
& \leq C \lambda^{2} \sum_{h=1}^{n_{\lambda}(\tau)-1} \sum_{k=1}^{h} \frac{\sqrt{T}}{(h-k+1)^{3 / 2}} \sup _{t \in\left[T_{k-1}, T_{k}\right]}\left\|z^{(k-1, \perp)}(t)\right\|_{\infty} \\
& \leq C T^{-1 / 2} \sup _{n \leq n_{\lambda}(\tau)} \sup _{t \in\left[T_{k-1}, T_{k}\right]}\left\|z^{(k-1, \perp)}(t)\right\|_{\infty} .
\end{aligned}
$$

On the other hand, for each $\gamma \in(0,1 / 2)$, since $\left\|\bar{m}_{x_{k-1}}^{\prime}\right\|_{1} \leq C$, we also have

$$
\begin{aligned}
\sup _{n \leq n_{\lambda}(\tau)} \frac{\left|\tilde{S}_{n}^{\|}(6,2)\right|}{1+x_{n, *}} & \leq C \lambda^{2} \sum_{h=1}^{n_{\lambda}(\tau)-1} \sum_{k=1}^{h} \int_{T_{h}}^{T_{h+1}} d s \int_{T_{k-1}}^{T_{k}} d s^{\prime} \frac{1}{\left(s-s^{\prime}\right)^{3 / 2+\gamma}}\left|w^{(k-1)}\left(s^{\prime}\right)\right| \\
& \leq C \lambda^{2} \sum_{h=1}^{n_{\lambda}(\tau)-1} \sum_{k=1}^{h} \frac{T^{1 / 2-\gamma}}{(h-k+1)^{3 / 2+\gamma}} \sup _{t \in\left[T_{k-1}, T_{k}\right]}\left|w^{(k-1)}(t)\right| \\
& \leq C T^{-\gamma} \sup _{n \leq n_{\lambda}(\tau)} \sup _{t \in\left[T_{k-1}, T_{k}\right]} \frac{\left|w^{(k)}(t)\right|}{\sqrt{T}} .
\end{aligned}
$$

Since $\left|w^{(k)}(t)\right| \leq C\left\|z^{(k-1)}(t)\right\|_{\infty}$ by using the Gaussian estimate (4.6) (resp. $(4.35))$ and $(7.21)$ (resp. in $(7.20))$, the bound in $(7.7)$ for $\tilde{S}_{n}(6,2)$ now follows.

The case $\ell=(9 \mid 2 ; 2)$. We have $S_{n}(9 \mid 2 ; 2)=\sum_{k=0}^{n-1} \eta_{k}(9 \mid 2 ; 2)$ where

$$
\eta_{k}(9 \mid 2 ; 2)=\frac{9 \lambda^{2}}{4} \int_{T_{k}}^{T_{k+1}} d t\left\langle\bar{m}_{x_{k}}^{\prime}, \bar{m}_{x_{k}} z^{(k)}(t)^{2}\right\rangle
$$


We define

$$
\gamma_{k}:=\mathbb{E}\left(\eta_{k}(9 \mid 2 ; 2) \mid \mathcal{F}_{T_{k}}\right)
$$

and then decompose

$$
S_{n}(9 \mid 2 ; 2)=M_{n}+\sum_{k=0}^{n-1} \gamma_{k}
$$

where $M_{n}$ is an $\mathcal{F}_{T_{n}}$-martingale with bracket

$$
\langle M\rangle_{n}=\sum_{k=0}^{n-1}\left[\mathbb{E}\left(\eta_{k}(9 \mid 2 ; 2)^{2} \mid \mathcal{F}_{T_{k}}\right)-\gamma_{k}^{2}\right]
$$

Note that the difference between the bracket $\langle\cdot\rangle_{n}$ and the quadratic variation $[\cdot]_{n}$ is a martingale, see [16].

We have

$$
\gamma_{k}=\frac{9 \lambda^{2}}{4} \int_{T_{k}}^{T_{k+1}} d t \int_{T_{k}}^{t} d s \int d x \int d y \bar{m}^{\prime}(x) \bar{m}(x) g_{t-s}(x, y)^{2}\left[a\left(\lambda^{\beta}\left(y+x_{k}\right)\right)^{2}-1\right]
$$

where we exploited the identity

$$
\int d x \int d y \bar{m}^{\prime}(x) \bar{m}(x) g_{t-s}(x, y)^{2}=\int d x \bar{m}^{\prime}(x) \bar{m}(x) g_{2(t-s)}(x, x)=0
$$

which holds because $x \mapsto g_{t}(x, x)$ is an even function of $x$.

We claim for each $q>\log _{\lambda^{-1}} T=(1 \wedge \beta) / 20$ there is $C=C(q)$ so that

$$
\left|\gamma_{k}\right| \leq C(\lambda T)^{2}\left\{\lambda^{\beta-q}+\lambda^{\beta} x_{k, *}+e^{-\lambda^{-q} / C}\right\} .
$$

By Taylor expansion

$$
\begin{aligned}
\left|a\left(\lambda^{\beta}\left(y+x_{k}\right)\right)^{2}-1\right| & \leq C\left\{\lambda^{\beta}\left(|y|+\left|x_{k}\right|\right) \mathbf{1}_{|y| \leq \lambda^{-q}}+\mathbf{1}_{|y|>\lambda^{-q}}\right\} \\
& \leq C\left\{\lambda^{\beta-q}+\lambda^{\beta} x_{k, *}+\mathbf{1}_{|y|>\lambda^{-q}}\right\} .
\end{aligned}
$$

We use the bound (7.28) in (7.26). There is $C$ so that, for any $t \in[0, T](T>1)$,

$$
\int_{0}^{t} d s \int d x \int d y \bar{m}^{\prime}(x)|\bar{m}(x)| g_{s}(x, y)^{2} \leq \int_{0}^{t} d s \int d x \bar{m}^{\prime}(x) g_{2 s}(x, x) \leq C T
$$

the first two terms on the r.h.s. of (7.28) produce the first two terms on the r.h.s. of (7.27). We estimate next the contribution of the last one. Denoting by $\mathbb{E}_{x, y, t}$ the expectation w.r.t. a Brownian bridge from $x$ to $y$ in time $t$, by the Feynman-Kac formula we get

$$
g_{t}(x, y)=p_{t}(x, y) \mathbb{E}_{x, y, t} \exp \left(-\int_{0}^{t} d s V^{\prime \prime}\left(\bar{m}\left(\omega_{s}\right)\right)\right) \leq e^{2 t} p_{t}(x, y)
$$


whence

$$
\begin{aligned}
\int d x \int d y & \mathbf{1}_{|x| \leq \lambda^{-q} / 2} \bar{m}^{\prime}(x) g_{t}(x, y)^{2} \mathbf{1}_{|y|>\lambda^{-q}} \\
\leq & \frac{e^{-\left[\lambda^{-2 q} /(8 t)\right]+2 t}}{\sqrt{2 \pi t}} \int d x \int d y g_{t}(x, y) \bar{m}^{\prime}(x)=2 \frac{e^{-\left[\lambda^{-2 q} /(8 t)\right]+2 t}}{\sqrt{2 \pi t}}
\end{aligned}
$$

On the other hand, since $\bar{m}^{\prime}(x)$ decays exponentially as $x \rightarrow \infty$,

$$
\begin{aligned}
\int d x \int d y \mathbf{1}_{|x|>\lambda-q / 2} \bar{m}^{\prime}(x) g_{t}(x, y)^{2} \mathbf{1}_{|y|>\lambda-q} & \leq C e^{-\lambda^{-q} / C} \int d x g_{2 t}(x, x) \sqrt{\bar{m}^{\prime}(x)} \\
& \leq C \frac{e^{-\lambda^{-q} / C+4 t}}{\sqrt{4 \pi t}}
\end{aligned}
$$

Taking $q>\log _{\lambda^{-1}} T$, from (7.30) and (7.31) we find that the last term on the r.h.s. of (7.28) yields the last one on the r.h.s. of (7.27).

From (7.27), for any $\lambda$ small enough,

$$
\sup _{n \leq n_{\lambda}(\tau)} \frac{1}{1+x_{n, *}}\left|\sum_{k=0}^{n-1} \gamma_{k}\right| \leq C T \lambda^{\beta-q} .
$$

By choosing $q \in\left(\log _{\lambda^{-1}} T, \beta-\log _{\lambda^{-1}} T\right)$ (recall we fixed $\log _{\lambda^{-1}} T=(1 \wedge \beta) / 20$ ) it follows the l.h.s. of (7.32) vanishes as $\lambda \downarrow 0$.

We are left with the bound on the martingale part $M_{n}$. By Doob's inequality, recalling (7.25),

$$
\begin{aligned}
\mathbb{P}\left(\sup _{n \leq n_{\lambda}(\tau)}\left|M_{n}\right|>T^{-1 / 4}\right) & \leq C \sqrt{T} \mathbb{E}\left(\langle M\rangle_{n_{\lambda}(\tau)}\right) \leq C \sqrt{T} \sum_{k=0}^{n_{\lambda}(\tau)} \mathbb{E}\left(\eta_{k}(9 \mid 2 ; 2)^{2}\right) \\
& \leq C \sqrt{T}\left(\lambda^{2} T\right)^{-1}\left(C \lambda^{2} T^{3 / 2}\right)^{2}
\end{aligned}
$$

in the second estimate we used (recall (7.9))

$$
\sqrt{\mathbb{E}\left(\eta_{k}(9 \mid 2 ; 2)^{2}\right)} \leq C \lambda^{2} \int_{T_{k}}^{T_{k+1}} d t \int d x \bar{m}^{\prime}(x) \sqrt{\mathbb{E}\left(z^{(k)}(t, x)^{4}\right)} \leq C \lambda^{2} T^{3 / 2} .
$$

From (7.32) and (7.33) (recall $x_{n, *}$ is increasing), (7.7) for $\ell=(9 \mid 2 ; 2)$ follows.

\section{Conclusion of the proof}

In this section we prove Theorem 1.1. As before we denote by $(m(t), h(t))$ the solution of (1.1)-(1.10) (omitting the dependence on $\lambda$ ). Let $\delta$ be as in Proposition 2.1 and define the stopping time

$$
t_{\delta}:=\inf \left\{t \geq 0: \inf _{z \in \mathbb{R}}\left\|m(t)-\bar{m}_{z}\right\|_{\infty} \geq \delta\right\}
$$


By Proposition 2.1, for each $t \in\left[0, t_{\delta}\right]$ the profile $m(t)$ has a well defined center $x(m(t))$. We prove the theorem with

$$
x^{(\lambda)}(t):=x\left(m\left(t \wedge t_{\delta}\right)\right) .
$$

Observe the process $x^{(\lambda)}(t)$ is adapted to $\mathcal{F}_{t}$. Moreover, by Proposition 2.1 and the continuity properties of $m(t)$, the process $x^{(\lambda)}(t)$ is continuous.

Proof of (1.11). Given $\tau, \zeta>0$, from Propositions 2.1, 4.1, and recalling (3.1) there is $C=C(\tau, \zeta)$ so that, for any $\lambda$ small enough,

$$
t_{\delta}>\lambda^{-2} \tau, \quad \sup _{n \leq n_{\lambda}(\tau)} \sup _{t \in\left[T_{n}, T_{n+1}\right]}\left|x^{(\lambda)}(t)-x_{n}\right| \leq C \lambda^{1-\zeta} \sqrt{T} \quad \text { on the set } \mathcal{B}_{\lambda, \tau}^{(1)} .
$$

On the other hand, by (3.1), (4.2), and $\left\|\bar{m}^{\prime}\right\|_{\infty} \leq 1$, we have

$$
\sup _{t \in\left[T_{n}, T_{n+1}\right]}\left\|m(t)-\bar{m}_{x^{(\lambda)}(t)}\right\|_{\infty} \leq V_{n}+\sup _{t \in\left[T_{n}, T_{n+1}\right]}\left|x^{(\lambda)}(t)-x_{n}\right| \quad \forall n: T_{n+1} \leq t_{\delta} .
$$

From (4.6), (4.7), (8.2), and (8.3), by choosing $\zeta$ small enough (1.11) follows.

To complete the proof of Theorem 1.1 we need to prove the weak convergence of the scaled process $x_{\lambda}(\tau):=x^{(\lambda)}\left(\lambda^{-2} \tau\right)$. Let $\xi_{\lambda}(\tau), \tau \in \mathbb{R}_{+}$, be the (continuous) process obtained by linearly interpolating the values $\xi_{\lambda}\left(\lambda^{2} T_{n}\right)=\xi_{n}$. From (4.6), (4.35), (4.39), and (8.2), for any $\tau$ and $\varepsilon$ positive,

$$
\lim _{\lambda \downarrow 0} \mathbb{P}\left(\sup _{0 \leq s \leq \tau}\left|\xi_{\lambda}(s)-x_{\lambda}(s)\right|>\varepsilon\right)=0 .
$$

It is therefore enough to prove the convergence of the process $\xi_{\lambda}$. To this end we shall use that $\xi_{n}$ solves the equation (7.2).

In Sections 7 and 8 we proved bounds on $R$ and $S$, see (7.4) and Proposition 7.3 which hold with probability going to 1 as $\lambda \downarrow 0$. They however depend on the unknown quantities $x_{n, *}$. The a priori bounds of Section 4 yield $x_{n, *} \leq n \delta^{*}(\tau)$, which goes like $\lambda^{-1} T^{-1 / 2+\zeta}$, according to (4.7) and with $n=n_{\lambda}(\tau)$. With such a bound, our estimates on $R$ and $S$ become very bad and in any case inadequate to study the equation (7.2), for which we could only tolerate a bound on $x_{n, *}$ which diverges very weakly as $\lambda \downarrow 0$.

As mentioned, the bounds of Section 4 on $\delta^{*}(\tau)$ are quasi optimal, yet they yield a bound on $x_{n, *}$ which is far from correct. The point is that $x_{n}$ is the sum of the increments $x_{k}-x_{k-1}$, whose size has indeed the order of $\delta^{*}(\tau)$; but there are a lot of cancellations, which make the absolute value of the sum much smaller than the sum of the absolute values. Such cancellations are lost using the a priori bounds of Section 4, we thus need to go back to the equation (7.2) itself (recalling that $\xi_{n}$ and $x_{n}$ are essentially the same, see (4.39)). It seems, at this point, that we are back to a non linear problem, with the unknown $\xi_{n}$ in both the "known terms" $R$ and $S$ hidden through $x_{n}$. Such a non linearity is however not really dangerous, 
as in our bounds for $R$ and $S$ the dependence on $x_{n, *}$ is always multiplied by a function of $\lambda$ which vanishes as $\lambda \downarrow 0$. As we will see, this makes easy the proof of Proposition 8.1 below.

Let

$$
\xi^{*}(\tau):=\sup _{n \leq n_{\lambda}(\tau)}\left|\xi_{n}\right|
$$

and $x_{*}(\tau)$ be as in $(6.5)$.

Proposition 8.1 For any $\tau \in \mathbb{R}_{+}$,

$$
\begin{aligned}
& \lim _{L \rightarrow \infty} \varlimsup_{\lambda \downarrow 0} \mathbb{P}\left(\xi^{*}(\tau)>L\right)=0, \\
& \lim _{L \rightarrow \infty} \varlimsup_{\lambda \downarrow 0} \mathbb{P}\left(x_{*}(\tau)>L\right)=0 .
\end{aligned}
$$

Proof. Since (8.6) follows from (8.5) and (4.39), we only need to prove (8.5). Let (recall $(7.1))$

$$
S_{n}^{(R)}:=\sum_{\ell \in \mathcal{L} \backslash\{2\}} S_{n}(\ell)
$$

and denote as usual by $S^{(R)}$ the vector whose components are $S_{n}^{(R)}$.

Given $\tau>0$ and $\zeta>0$ small enough, let $\mathcal{B}_{\lambda, \tau}$ be as in (6.6) and define

$$
\mathcal{G}_{\lambda, L}:=\mathcal{A}_{\lambda, L} \cap \mathcal{B}_{\lambda, \tau}
$$

where $\mathcal{A}_{\lambda, L}$ is the event

$$
\mathcal{A}_{\lambda, L}:=\left\{\omega \in \Omega:|S(2)|_{n_{\lambda}(\tau)} \leq L ; \sup _{n \leq n_{\lambda}(\tau)} \frac{\left|S^{(R)}\right|_{n}}{1+x_{n, *}} \leq T^{-1 / 4}\right\} .
$$

From (4.6), (4.35), (6.6), (6.4), and Proposition 7.3 we have, for any $\tau>0$,

$$
\lim _{L \rightarrow \infty} \frac{\lim }{\lambda \downarrow 0} \mathbb{P}\left(\mathcal{G}_{\lambda, L}\right)=1 .
$$

We are going to show that given $L$ there is a constant $c$ so that, for all $\lambda$ small enough, in $\mathcal{G}_{\lambda, L}$ we have $\left|\xi_{n}\right| \leq c$ for all $n \leq n_{\lambda}(\tau)+1$.

Given any $L_{1}>0$, let $N\left(L_{1}, \lambda\right)$ be the first index $n \leq n_{\lambda}(\tau)$ for which $\left|\xi_{n}\right| \geq L_{1}$; otherwise we set $N\left(L_{1}, \lambda\right)=n_{\lambda}(\tau)+1$. Then what we have to show is that there is an $L_{1}$ so that for all $\lambda$ small enough, $N\left(L_{1}, \lambda\right)=n_{\lambda}(\tau)+1$ in $\mathcal{G}_{\lambda, L}$. Having fixed $L_{1}$, there is a $\lambda_{1}$ so that for $\lambda \leq \lambda_{1}$ and $n \leq N\left(L_{1}, \lambda\right)$, by (4.39) (recall $\mathcal{G}_{\lambda, L} \subseteq \mathcal{B}_{\lambda, \tau}^{(1,2)}$ )

$$
\left|x_{n}\right| \leq\left|\xi_{n}\right|+1 \leq L_{1}+1, \quad \text { on the set } \mathcal{G}_{\lambda, L} .
$$


Using this, (7.4), and (8.9) we then get there is $\lambda_{2}=\lambda_{2}\left(\lambda_{1}, L_{1}\right) \in\left(0, \lambda_{1}\right)$ such that for any $\lambda \leq \lambda_{2}$ and $n \leq N\left(L_{1}, \lambda\right)$,

$$
|R|_{n} \leq 1, \quad|S|_{n} \leq L+1, \quad \text { on the set } \mathcal{G}_{\lambda, L} .
$$

Then, by (7.2) and (5.19), for $n \leq N\left(L_{1}, \lambda\right)$,

$$
\left|\xi_{n}\right| \leq C(L+1)+C \sum_{k=0}^{n-1} \lambda^{2} T\left|\xi_{k}\right|, \quad \text { on the set } \mathcal{G}_{\lambda, L} .
$$

By solving this inequality, we conclude that there is a $c=c(C, L, \tau)$, independent of $L_{1}$, so that $\left|\xi_{n}\right| \leq c$, for all $n \leq N\left(L_{1}, \lambda\right)$. By taking $L_{1} \geq c$, we have $N\left(L_{1}, \lambda\right)>$ $n_{\lambda}(\tau)$. Proposition 8.1 is proved.

Setting

$$
Y_{n}:=S_{n}(2)-\sum_{k=0}^{n-1} A_{n, k} S_{k}(2)
$$

from (7.4), (8.6), (8.10), and $\|A\|_{n} \leq C$, we have, for each $\varepsilon>0$,

$$
\lim _{\lambda \downarrow 0} \mathbb{P}\left(\sup _{n \leq n_{\lambda}(\tau)}|S-A S+R-Y|_{n}>\varepsilon\right)=0
$$

so that the analysis of the limiting behavior of the "known term" $S-A S+R$ in (7.2) reduces to that of $Y$, which is the content of the following proposition.

Proposition 8.2 Let $Y_{\lambda}(\tau), \tau \in \mathbb{R}_{+}$, be the process obtained by linearly interpolating the values $Y_{\lambda}\left(\lambda^{2} T_{n}\right)=Y_{n}, n \in \mathbb{N}$. Then

$$
Y_{\lambda}(\tau) \stackrel{\lambda \downarrow 0}{\Longrightarrow} b(\tau)-\frac{3}{\sqrt{2 \pi}} \int_{0}^{\tau} d s \frac{b(s)}{\sqrt{\tau-s}}
$$

where the convergence is in $C\left(\mathbb{R}_{+}\right)$, and $b(\tau)$ is a one dimensional Brownian motion with diffusion coefficient $D=3 / 4$.

Proof. We prove first that $(S(2))_{\lambda}(\tau)$ (obtained by linear interpolation from $S_{n}(2)$ ) converges weakly to a Brownian motion. We first prove tightness. Boundedness has already been proved, see (7.6). Since $S_{n}(2)$ is a $\mathcal{F}_{T_{n}}$-martingale, by Doob's inequality, for each $\varepsilon>0$, we have

$$
\begin{array}{r}
\lim _{\delta \downarrow 0} \varlimsup_{\lambda \downarrow 0} \mathbb{P}\left(\sup _{\substack{0 \leq \sigma<\tau \leq T \\
\tau-\sigma \leq \delta}}\left|(S(2))_{\lambda}(\tau)-(S(2))_{\lambda}(\sigma)\right|>\varepsilon\right) \\
\leq \lim _{\delta \downarrow 0} \varlimsup_{\lambda \downarrow 0} \frac{C\left[n_{\lambda}(\delta)+1\right] \lambda^{2} T}{\varepsilon^{2}}=0 .
\end{array}
$$


To identify the limit we compute the bracket of $S_{n_{\lambda}(\tau)}(2)$ as follows

$$
\begin{aligned}
\sum_{k=1}^{n_{\lambda}(\tau)} \mathbb{E}\left(\left(S_{k}(2)-S_{k-1}(2)\right)^{2} \mid \mathcal{F}_{T_{k-1}}\right) & =\frac{9 \lambda^{2}}{16} \sum_{k=1}^{n_{\lambda}(\tau)} \mathbb{E}\left(\left\langle\bar{m}_{x_{k-1}}^{\prime}, z^{(k-1)}\left(T_{k}\right)\right\rangle^{2} \mid \mathcal{F}_{T_{k-1}}\right) \\
& =\frac{9 \lambda^{2} T}{16} \sum_{k=1}^{n_{\lambda}(\tau)} \int d x \bar{m}^{\prime}(x)^{2} a\left(\lambda^{\beta}\left(x+x_{k-1}\right)\right)^{2}
\end{aligned}
$$

which converges to $D \tau$ on the set $\left\{x^{*}(\tau) \leq L\right\}$ for any $L>0$. From (8.6) and routine manipulation, $(S(2))_{\lambda}$ converges weakly to a Brownian motion with diffusion $D$ by Levy's characterization theorem.

To complete the proof, we introduce the family of linear mappings $J_{\lambda}$ : $C\left(\mathbb{R}_{+}\right) \rightarrow C\left(\mathbb{R}_{+}\right)$defined by

$$
J_{\lambda}(\varphi)(\tau):=\sum_{k=0}^{n_{\lambda}(\tau)-1} A_{n_{\lambda}(\tau)-1, k-1} \varphi\left(\lambda^{2} T k\right)
$$

when $\left(\lambda^{2} T\right)^{-1} \tau$ is an integer and by linear interpolation otherwise. It is easy to verify that

$$
\lim _{\lambda \downarrow 0} J_{\lambda}(\varphi)(\tau)=J(\varphi)(\tau):=\frac{3}{\sqrt{2 \pi}} \int_{0}^{\tau} d s \frac{\varphi(s)}{\sqrt{\tau-s}}
$$

uniformly for $\varphi$ in a compact set. Since $J$ is continuous, we can apply [6, Thm. 5.3] and get

$$
J_{\lambda}\left((S(2))_{\lambda}\right)(\tau) \stackrel{\lambda \downarrow 0}{\Longrightarrow} \frac{3}{\sqrt{2 \pi}} \int_{0}^{\tau} d s \frac{b(s)}{\sqrt{\tau-s}} \quad \text { in } C\left(\mathbb{R}_{+}\right) .
$$

Proposition 8.2 is proved.

Conclusion of the proof of Theorem 1.1. We first show that $\xi_{\lambda}$ converges by subsequences to a continuous process, and then that any limit point solves the integral equation (1.12). By the uniqueness (in law) of the latter, Theorem 1.1 follows.

The boundedness of $\xi_{\lambda}$ follows from (8.5). In order to prove equicontinuity, we note that from (7.2) and (8.14) we have, for each $\varepsilon>0$,

$$
\lim _{\lambda \downarrow 0} \mathbb{P}\left(\sup _{n \leq n_{\lambda}(\tau)}\left|\xi_{n}-Y_{n}-\sum_{k=0}^{n-1}\left(A^{2}\right)_{n, k} \xi_{k}\right|>\varepsilon\right)=0 .
$$

Setting

$$
\Xi_{\lambda}(s):=\sum_{k=0}^{n_{\lambda}(s)-1}\left(A^{2}\right)_{n_{\lambda}(s), k} \xi_{k}
$$


we postpone the proof that, for each $\varepsilon>0$,

$$
\lim _{\delta \downarrow 0} \varlimsup_{\lambda \downarrow 0} \mathbb{P}\left(\sup _{\substack{s, s^{\prime} \in[0, \tau] \\\left|s-s^{\prime}\right| \leq \delta}}\left|\Xi_{\lambda}(s)-\Xi_{\lambda}\left(s^{\prime}\right)\right|>\varepsilon\right)=0
$$

and observe that, by (8.16), Proposition 8.2, and (8.18), for each $\varepsilon>0$,

$$
\lim _{\delta \downarrow 0} \varlimsup_{\lambda \downarrow 0} \mathbb{P}\left(\sup _{\substack{s, s^{\prime} \in[0, \tau] \\\left|s-s^{\prime}\right| \leq \delta}}\left|\xi_{\lambda}(s)-\xi_{\lambda}\left(s^{\prime}\right)\right|>\varepsilon\right)=0
$$

which shows $\xi_{\lambda}$ is tight in $C\left(\mathbb{R}_{+}\right)$.

It remains to prove (8.18). Let us fix $0 \leq s^{\prime} \leq s \leq \tau, s-s^{\prime} \leq \delta$. We have

$$
\begin{aligned}
\Xi_{\lambda}(s)-\Xi_{\lambda}\left(s^{\prime}\right)= & \sum_{k=0}^{n_{\lambda}\left(s^{\prime}-\delta\right)-1}\left(\left(A^{2}\right)_{n_{\lambda}(s), k}-\left(A^{2}\right)_{n_{\lambda}\left(s^{\prime}\right), k}\right) \xi_{k} \\
& +\sum_{k=n_{\lambda}\left(s^{\prime}-\delta\right)}^{n_{\lambda}\left(s^{\prime}\right)-1}\left(\left(A^{2}\right)_{n_{\lambda}(s), k}-\left(A^{2}\right)_{n_{\lambda}\left(s^{\prime}\right), k}\right) \xi_{k} \\
& +\sum_{k=n_{\lambda}\left(s^{\prime}\right)}^{n_{\lambda}(s)-1}\left(A^{2}\right)_{n_{\lambda}(s), k} \xi_{k}
\end{aligned}
$$

so that

$$
\begin{aligned}
\left|\Xi_{\lambda}(s)-\Xi_{\lambda}\left(s^{\prime}\right)\right| \leq & 2 \xi^{*}(\tau) n_{\lambda}(\tau) \lambda^{2} T \sup _{\substack{\tau_{1}, \tau_{2} \in[0, \tau] \\
\tau_{1}-\tau_{2}>\delta}}\left|\left(\lambda^{2} T\right)^{-1}\left(A^{2}\right)_{n_{\lambda}\left(\tau_{1}\right), n_{\lambda}\left(\tau_{2}\right)}-\frac{9}{2}\right| \\
& +3 \xi^{*}(\tau) n_{\lambda}(\delta) \sup _{0 \leq k<n \leq n_{\lambda}(\tau)}\left|\left(A^{2}\right)_{n, k}\right|
\end{aligned}
$$

by using (5.19), (5.20), and (8.5) the equation (8.18) follows. We have concluded the proof that $\xi_{\lambda}$ is tight.

Finally, by (5.20), Proposition 8.2 and (8.16), we conclude that any limit $\xi$ solves

$$
\xi(\tau)=b(\tau)-\frac{3}{\sqrt{2 \pi}} \int_{0}^{\tau} d s \frac{b(s)}{\sqrt{2 \pi(\tau-s)}}+\frac{9}{2} \int_{0}^{\tau} d s \xi(s) .
$$

It is now easy to verify (1.12) and (8.20) are equivalent; indeed the latter is obtained by an iteration of the former. 


\section{Appendices}

\section{A Some technical estimates}

We have $p_{t}(x, y)=p_{t}(x-y, 0)$ and

$$
\frac{\partial p_{t}(x, 0)}{\partial t}=\frac{1}{\sqrt{\pi}(2 t)^{3 / 2}}\left[\frac{x^{2}}{t}-1\right] e^{-x^{2} / 2 t} .
$$

Proof of (4.17).

$$
\left|\frac{\partial}{\partial t}\left(p_{t} \varphi\right)(x)\right| \leq\|\varphi\|_{\infty} \frac{1}{2 t} \int d y p_{t}(y, 0)\left[1+\frac{y^{2}}{t}\right]=\frac{1}{t}\|\varphi\|_{\infty} .
$$

Proof of (7.19).

$$
\begin{aligned}
\left\langle\bar{m}^{\prime \prime}, \frac{\partial p_{t}}{\partial t} \varphi\right\rangle & =-\left\langle\bar{m}^{\prime}, \frac{\partial^{2} p_{t}}{\partial x \partial t} \varphi\right\rangle \\
& =\int d x \bar{m}^{\prime}(x) \int d y \frac{1}{\sqrt{\pi}(2 t)^{3 / 2}}\left[\frac{(x-y)^{3}}{t^{2}}-\frac{3(x-y)}{t}\right] e^{-(x-y)^{2} / 2 t} \varphi(y) \\
& =\frac{1}{2 t^{3 / 2}} \int d x \bar{m}^{\prime}(x) \int d y p_{t}(x, y)\left[\frac{(x-y)^{3}}{t^{3 / 2}}-\frac{3(x-y)}{\sqrt{t}}\right] \varphi(y)
\end{aligned}
$$

hence, by the Young and Hölder inequalities, (7.19) follows.

\section{B Gaussian estimates}

Proof of (7.9). From (1.3) and (3.9) we have, for $t \in\left[T_{n}+1, T_{n+1}\right]$

$$
\mathbb{E}\left(z^{(n)}(t, x)^{2} \mid x_{n}\right)=\int_{T_{n}}^{t} d s \int d y g_{t-s}^{(n)}(x, y)^{2} a\left(\lambda^{\beta} y\right)^{2} \leq C \int_{0}^{t-T_{n}} d u g_{2 u}^{(n)}(x, x) .
$$

Next, since $\sup \left\{g_{u}^{(n)}(x, y): x, y \in \mathbb{R} ; 2 u \geq 1\right\}<\infty$ (see [7, Lemma A.9]), and using also (7.29) the desired estimate holds for $p=1$. Since the process $z^{(n)}$ conditioned on $x_{n}$ is Gaussian, the estimate also holds for $p=2$.

Proof of (4.6). Let us denote by $\mathbb{P}_{n}$ the probability $\mathbb{P}$ conditioned on the center $x_{n}$, and by $\mathbb{E}_{n}$ the corresponding expectation.

From (B.1) and (7.29) to treat the case $T_{n} \leq t \leq T_{n}+1$, it follows there exists $C>0$ such that, for any $n \leq n_{\lambda}(\tau)$,

$$
\sup _{t \in\left[T_{n}, T_{n+1}\right]} \sup _{x \in \mathbb{R}} \mathbb{E}_{n}\left(z^{(n)}(t, x)^{2}\right) \leq C T .
$$


Proceeding as in the proof of [7, Thm. 2.3], (taking $\varepsilon=1$ and $\mu(y)=a\left(\lambda^{\beta} y\right)$ ), it follows that given $\alpha \in(0,1)$ there exists $C$ such that, for any $h, \lambda \in(0,1]$, $t \in\left[T_{n}, T_{n+1}\right]$, and $x \in \mathbb{R}$,

$$
\mathbb{E}_{n}\left(\left(z^{(n)}(t, x+h)-z^{(n)}(t, x)\right)^{2}\right) \leq C T h^{\alpha} .
$$

It is not difficult to prove also Hölder continuity in $t$ :

$$
\mathbb{E}_{n}\left(\left(z^{(n)}(t+h, x)-z^{(n)}(t, x)\right)^{2}\right) \leq C T^{2} \lambda^{-\beta} \sqrt{h} .
$$

Indeed recall that, from (1.3),

$$
\begin{aligned}
\mathbb{E}_{n}\left(\left(z^{(n)}(t+h, x)-\right.\right. & \left.\left.z^{(n)}(t, x)\right)^{2}\right)= \\
& \int_{T_{n}}^{t} d s \int d y\left(g_{t-s+h}^{(n)}(x, y)-g_{t-s}^{(n)}(x, y)\right)^{2} a\left(\lambda^{\beta} y\right)^{2} \\
& +\int_{0}^{h} d s \int d y g_{s}^{(n)}(x, y)^{2} a\left(\lambda^{\beta} y\right)^{2} .
\end{aligned}
$$

Using (7.29) it is easy to see that

$$
\int_{0}^{h} d s \int d y g_{s}^{(n)}(x, y)^{2} a\left(\lambda^{\beta} y\right)^{2} \leq C \sqrt{h} .
$$

To bound the first term on the r.h.s. of (B.5), we use the following formula for $g_{t}^{(n)}$ :

$$
g_{t}^{(n)}(x, y)=p_{t}(x, y)+\int_{0}^{t} d s \int d z p_{t-s}(x, z)\left[1-3 \bar{m}_{x_{n}}^{2}(z)\right] g_{s}^{(n)}(z, y) .
$$

From the properties of the heat kernel $p_{t}$, the estimate $\sup \left\{g_{t}^{(n)}(x, y) ; x, y \in \mathbb{R}, t \geq\right.$ $1\}<\infty$, the fact that $a$ has compact support and (7.29) for $t \in(0,1]$, one gets (B.4).

Recall that $z_{n}$ conditioned on $x_{n}$ is Gaussian, and let us now define

$$
\begin{aligned}
\sigma_{n}^{2} & =\sup _{t \in\left[T_{n}, T_{n+1}\right]} \sup _{x \in \mathbb{R}} \mathbb{E}_{n}\left(z^{(n)}(t, x)^{2}\right) \\
\mu_{n} & =\mathbb{E}_{n}\left(\sup _{t \in\left[T_{n}, T_{n+1}\right]}\left\|z^{(n)}(t)\right\|_{\infty}\right) .
\end{aligned}
$$

Borell's inequality (see for instance [1, Thm. 2.1]) tells us that, if $\mu_{n}<$ $\sqrt{T} \lambda^{-\zeta}$, then

$$
\mathbb{P}_{n}\left(\sup _{t \in\left[T_{n}, T_{n+1}\right]}\left\|z^{(n)}(t)\right\|_{\infty}>\lambda^{-\zeta} \sqrt{T}\right) \leq 4 \exp \left[-\frac{\left(\lambda^{-\zeta} \sqrt{T}-\mu_{n}\right)^{2}}{2 \sigma_{n}^{2}}\right] .
$$


To estimate $\mu_{n}$, we use (see [1, Cor. 4.15]),

$$
\mu_{n} \leq K \int_{0}^{\infty} d r \sqrt{\log N(r)}
$$

where $K$ is a universal constant and $N(r)$ is the minimal number of balls of radius $r$ needed to cover $\left[T_{n}, T_{n+1}\right] \times \mathbb{R}$ when considering in that set the metric

$$
d((t, x),(s, y))=\sqrt{\mathbb{E}_{n}\left(\left(z^{(n)}(t, x)-z^{(n)}(s, y)\right)^{2}\right)} .
$$

Let us then estimate $N(r)$. Recall that, from (B.2), we only need to consider $r \leq \sqrt{C T}$, since for larger $r, N(r)=1$ and the integrand in (B.10) equals zero. For each $r>0$, consider the sets $A_{1}=\left[T_{n}, T_{n+1}\right] \times\left[-\left(\lambda^{2 \beta} r\right)^{-1},\left(\lambda^{2 \beta} r\right)^{-1}\right]$ and $A_{2}=\left(\left[T_{n}, T_{n+1}\right] \times \mathbb{R}\right) \backslash A_{1}$. Let us estimate first the number of balls needed to cover $A_{2}$.

From now on, to avoid introducing new constants in the notation we suppose that the support of $a$ is included in $[-1,1]$, and that $\lambda$ is sufficiently small. From (7.29), and since $T_{n+1}-T_{n}=T \leq \lambda^{-\beta / 20}$ and $r \leq \sqrt{C T}$, it is easy to see that if $(t, x) \in A_{2}$

$$
\begin{aligned}
\mathbb{E}_{n}\left(z^{(n)}(t, x)^{2}\right) & \leq \int_{T_{n}}^{t} d s \int d y e^{4(t-s)} p_{t-s}(x, y)^{2} a\left(\lambda^{\beta} y\right)^{2} \\
& \leq C e^{4 T} \int_{T_{n}}^{t} d s \frac{1}{\sqrt{t-s}} \exp \left[-\frac{\left(\left(\lambda^{2 \beta} r\right)^{-1}-\lambda^{-\beta}\right)^{2}}{2(t-s)}\right] \\
& \leq \exp \left[-\lambda^{-\beta} /\left(2 r^{2}\right)\right] \leq \frac{r^{2}}{4} .
\end{aligned}
$$

From (B.11), it follows that we may cover $A_{2}$ with just one ball. From (B.3) and (B.4), it follows that $A_{1}$ may be covered by $\left(\lambda^{7 \beta} r^{9}\right)^{-1}$ balls of radius $r$. Then, from (B.10) it follows that

$$
\begin{aligned}
\mu_{n} & \leq K \int_{0}^{\infty} d r \sqrt{\log N(r)} \leq K \int_{0}^{\sqrt{C T}} d r \sqrt{\log \left(1+\left(\lambda^{7 \beta} r^{9}\right)^{-1}\right)} \\
& \leq K \lambda^{-7 \beta / 9} \int_{\frac{\lambda-7 \beta / 9}{\sqrt{C T}}}^{\infty} d u \frac{\sqrt{\log \left(1+u^{9}\right)}}{u^{2}} \leq K \lambda^{-7 \beta / 9} \int_{\frac{\lambda-7 \beta / 9}{\sqrt{C T}}}^{\infty} d u \frac{9 \log u}{u^{2}} \\
& \leq C(\beta) \sqrt{T}|\log \lambda|
\end{aligned}
$$

where we have done the change of variables $u=\lambda^{-7 \beta / 9} r^{-1}$, and $C(\beta)$ is a constant that depends on $\beta$. Then, we may apply (B.9), to obtain

$$
\mathbb{P}_{n}\left(\sup _{t \in\left[T_{n}, T_{n+1}\right]}\left\|z^{(n)}(t)\right\|_{\infty}>\lambda^{-\zeta} \sqrt{T}\right) \leq 4 \exp \left(-C \lambda^{-\zeta / 2}\right) .
$$


Finally, recall that, given $x_{n}$, the random variables

$$
\left\{\sup _{t \in\left[T_{n}, T_{n+1}\right]}\left\|z^{(n)}(t)\right\|_{\infty} ; n \leq n_{\lambda \tau}\right\}
$$

are independent, and the estimate (B.13) does not depend on $n$ (in particular, it does not depend on $x_{n}$ ). Then

$$
\mathbb{P}\left(\mathcal{B}_{\lambda, \tau}^{(1)}\right) \geq\left(1-4 \exp \left(-C \lambda^{-\zeta / 2}\right)\right)^{n_{\lambda}(\tau)}
$$

and (4.6) follows.

Proof of (4.35). The estimate (4.35) follows by the same procedure used to prove (4.6). We use the notation introduced in that proof. Recall that

$$
z^{n, \perp}(t)=\int_{T_{n}}^{t} g_{t-s}^{n, \perp} d W^{(a)}(s)
$$

and define

$$
\begin{aligned}
& \sigma_{(n, \perp)}^{2}=\sup _{t \in\left[T_{n}, T_{n+1}\right] x \in \mathbb{R}} \sup _{n} \mathbb{E}_{n}\left(z^{(n, \perp)}(t, x)^{2}\right) \\
& \mu_{(n, \perp)}=\mathbb{E}_{n}\left(\sup _{t \in\left[T_{n}, T_{n+1}\right]}\left\|z^{(n, \perp)}(t)\right\|_{\infty}\right) .
\end{aligned}
$$

Then,

$$
\begin{aligned}
\mathbb{E}_{n}\left(z^{(n, \perp)}(t, x)^{2}\right) & =\int_{T_{n}}^{t} d s \int d y\left(g_{t-s}^{(n)}(x, y)-\frac{3}{4} \bar{m}_{x_{n}}^{\prime}(x) \bar{m}_{x_{n}}^{\prime}(y)\right)^{2} a\left(\lambda^{\beta} y\right)^{2} \\
& \leq\|a\|_{\infty} \int_{0}^{\infty} d s \int d y\left(g_{t-s}(x, y)-\frac{3}{4} \bar{m}^{\prime}(x) \bar{m}^{\prime}(y)\right)^{2} \\
& =\|a\|_{\infty} \int_{0}^{\infty} d s\left(g_{2 t}(x, x)-\frac{3}{4} \bar{m}^{\prime}(x)^{2}\right) \leq C
\end{aligned}
$$

(see [8] for the last inequality). It is easy to see that the estimates (B.3) and (B.4) also hold for $z^{(n, \perp)}$. Moreover,

$$
\mathbb{E}_{n}\left(z^{(n, \perp)}(t, x)^{2}\right) \leq C \mathbb{E}_{n}\left(z^{(n)}(t, x)^{2}\right)+C t \bar{m}^{\prime}(x)^{2}
$$

(see (4.33)), hence from (B.11) and recalling that $\bar{m}^{\prime}$ is exponentially small as $|x| \rightarrow \infty$, it follows that, for $\lambda$ small enough,

$$
\mathbb{E}_{n}\left(z^{(n, \perp)}(t, x)^{2}\right) \leq \frac{r^{2}}{4} \quad \text { for }(t, x) \in A_{2} .
$$


Then $N(r) \leq 1+\left(\lambda^{7 \beta} r^{9}\right)^{-1}$, so proceeding as in (B.12), but now with $T=1$, we obtain

$$
\mu_{n, \perp} \leq C|\log \lambda|
$$

and the proof finishes as that of (4.6).

Proof of (6.4). Note that, conditionally on the $x_{k-1}$ 's, the random variables $\left\{\eta_{k}(2) ; k \leq n_{\lambda}(\tau)\right\}$ are independent and Gaussian with variance bounded by $C T$. Therefore (6.4) is easily deduced from the following elementary lemma.

Lemma B.1 Let $\left\{\omega_{h}, h=1, \ldots, N\right\}$ be mean zero i.i.d. random variables and set $Y_{n}:=\sum_{h=1}^{n}(n-h+1)^{-1 / 2} \omega_{h}$. Assume that for each $p \in[1, \infty)$ we have $\mathbf{E}\left|\omega_{i}\right|^{p}<$ $\infty$. Then for each $\zeta>0$ and $q<\infty$ there exists a constant $C=C(\zeta, q)$ such that

$$
\mathbf{P}\left(\sup _{n \leq N}\left|Y_{n}\right|>N^{\zeta}\right) \leq C N^{-q}
$$

Proof. Let $Y_{k}^{(n)}:=\sum_{h=1}^{k}(n-h+1)^{-1 / 2} \omega_{h}, k=1, \ldots, n$, which is a martingale with quadratic variation

$$
\left[Y^{(n)}\right]_{k}=\sum_{h=1}^{k} \frac{1}{n-h+1} \omega_{h}^{2}
$$

hence, by the BDG inequality (see $[16, \S 6$, E. 4.1]), for each $p \in[1, \infty)$ there exists $C=C(p)$ such that

$$
\begin{aligned}
\mathbf{E}\left(\left|Y_{n}\right|^{p}\right) & =\mathbf{E}\left(\left|Y_{n}^{(n)}\right|^{p}\right) \leq C \mathbf{E}\left(\left(\left[Y^{(n)}\right]_{n}\right)^{p / 2}\right) \leq C \mathbf{E}\left(\sum_{h=1}^{n} \frac{1}{n-h+1} \omega_{h}^{2}\right)^{p / 2} \\
& \leq C\left[\sum_{h=1}^{n} \frac{1}{n-h+1}\left(\mathbf{E}\left|\omega_{h}\right|^{p}\right)^{2 / p}\right]^{p / 2} \leq C\left(\sum_{k=0}^{n-1} \frac{1}{k+1}\right)^{p / 2} \leq C(\log n)^{p / 2}
\end{aligned}
$$

By using (7.17) we thus get

$$
\mathbf{P}\left(\sup _{n \leq N}\left|Y_{n}\right|>N^{-\zeta}\right) \leq N^{-\zeta p} \sum_{n=1}^{N} \mathbf{E}\left(\left|Y_{n}\right|^{p}\right) \leq C N^{1-\zeta p}(\log N)^{p / 2}
$$

taking $p$ large enough the lemma follows.

Acknowledgments. S. Brassesco acknowledges the very kind hospitality of the Department of Mathematics of the University of Rome Tor Vergata. Part of this work was done while P. Buttà was visiting that Department with a Fellowship in Mathematics of the "Istituto Nazionale di Alta Matematica Francesco Severi". 


\section{References}

[1] R. J. Adler, An Introduction to Continuity, Extrema and Related Topics for General Gaussian Processes, Lecture Notes Monograph series 12, Institute of Mathematical Statistics 1990.

[2] L. Bertini, S. Brassesco, P. Buttà and E. Presutti, Stochastic phase field equations: existence and uniqueness, Ann. Henri Poincaré 3, 87-98 (2002).

[3] L. Bertini, P. Buttà, E. Presutti and E. Saada, Interface fluctuations in a conserved system: derivation and long time behavior, Preprint.

[4] L. Bertini, P. Buttà and B. Rüdiger, Interface dynamics and Stefan problem from a microscopic conservative model, Rend. Mat. Appl. (7) 19, 547-581 (1999).

[5] L. Bertini, E. Presutti, B. Rüdiger and E. Saada, Dynamical fluctuations at the critical point: convergence to a nonlinear stochastic PDE, Teor. Veroyatnost. i Primenen 38, no. 4, 689-741 (1993); Translation in Theory Probab. Appl. 38, no. 4, 586-629 (1993).

[6] P. Billingsley, Convergence of Probability Measures, New York: Wiley 1968.

[7] S. Brassesco, Stability of the Instanton under small random perturbations, Stoch. Proc. Appl. 54, 309-330 (1994).

[8] S. Brassesco and P. Buttà, Interface fluctuations for the $\mathrm{D}=1$ Stochastic Ginzburg-Landau equation with non-symmetric reaction term, J. Statist. Phys. 93, 1111-1142 (1998).

[9] S. Brassesco, P. Buttà, A. De Masi and E. Presutti, Interface fluctuations and couplings in the $d=1$ Ginzburg-Landau equation with noise, J. Theoret. Probab. 11, 25-80 (1998).

[10] S. Brassesco, A. De Masi and E. Presutti, Brownian fluctuations of the interface in the $d=1$ Ginzburg-Landau equation with noise, Annal. Inst. $H$. Poincaré 31, 81-118 (1995).

[11] G. Caginalp and X. Chen, Convergence of the phase field model to its sharp interface limits, European J. Appl. Math. 9, 417-445 (1998).

[12] A. De Masi, P. Ferrari and J. L. Lebowitz, Reaction-diffusion equations for interacting particle systems, J. Statist. Phys. 44, 589-644 (1986).

[13] T. Funaki, The scaling limit for a stochastic PDE and the separation of phases, Prob. Theory Relat. Fields 102, 221-288 (1995) .

[14] P. C. Hohenberg and B. I. Halperin, Theory of dynamic critical phenomena, Rev. Mod. Phys. 49, 435-479 (1977). 
[15] P. L. Lions and P. E. Souganidis, Fully nonlinear stochastic partial differential equations: non-smooth equations and applications, C. R. Acad. Sci. Paris Sér. I Math. 327, 735-741 (1998).

[16] M. Métivier, Semimartingales: A Course on Stochastic Processes, Berlin: de Gruyter 1982.

[17] H. M. Soner, Convergence of the phase-field equations to the Mullins-Sekerka problem with kinetic undercooling, Arch. Rational Mech. Anal. 131, 139-197 (1995).

[18] F. G. Tricomi, Integral Equations, New York: Dover 1985.

\section{Lorenzo Bertini}

Dipartimento di Matematica

Università di Roma La Sapienza

Piazzale Aldo Moro 2

00185 Roma

Italy

email: lorenzo@carpenter.mat.uniroma1.it

Stella Brassesco

Departamento de Matemáticas

Instituto Venezolano de Investigaciones Científicas

Apartado Postal 21827

Caracas 1020-A

Venezuela

email: sbrasses@ivic.ivic.ve

Paolo Buttà

Dipartimento di Matematica

Università di Roma La Sapienza

Piazzale Aldo Moro 2

00185 Roma

Italy

email: butta@mat.uniroma1.it

Errico Presutti

Dipartimento di Matematica

Università di Roma Tor Vergata

Via della Ricerca Scientifica

00133 Roma

Italy

email: presutti@mat.uniroma2.it

Communicated by Jean-Pierre Eckmann

submitted 11/01/01, accepted 13/06/01 